UNIVERSIDADE DE BRASÍLIA

FACULDADE DE TECNOLOGIA

DEPARTAMENTO DE ENGENHARIA MECÂNICA

\title{
MÉTODO DOS ELEMENTOS DE CONTORNO ISOGEOMÉTRICOS ACELERADO PELA APROXIMAÇÃO CRUZADA ADAPTATIVA
}

LUCAS SILVEIRA CAMPOS

ORIENTADOR: ÉDER LIMA DE ALBUQUERQUE

DOUTORADO EM CIÊNCIAS MECÂNICAS

PUBLICAÇÃO: ENM.TD - 36/2016

BRASÍLIA/DF: JULHO-2016 
UNIVERSIDADE DE BRASÍLIA

FACULDADE DE TECNOLOGIA

DEPARTAMENTO DE ENGENHARIA MECÂNICA

\section{MÉTODO DOS ELEMENTOS DE CONTORNO ISOGEOMÉTRICOS RÁPIDO}

\section{LUCAS SILVEIRA CAMPOS}

Relatório submetido como requisito parcial para obtenção do grau de Doutor em Ciências Mecânicas pelo Departamento de Engenharia Mecânica da Universidade de Brasília.

Banca Examinadora:
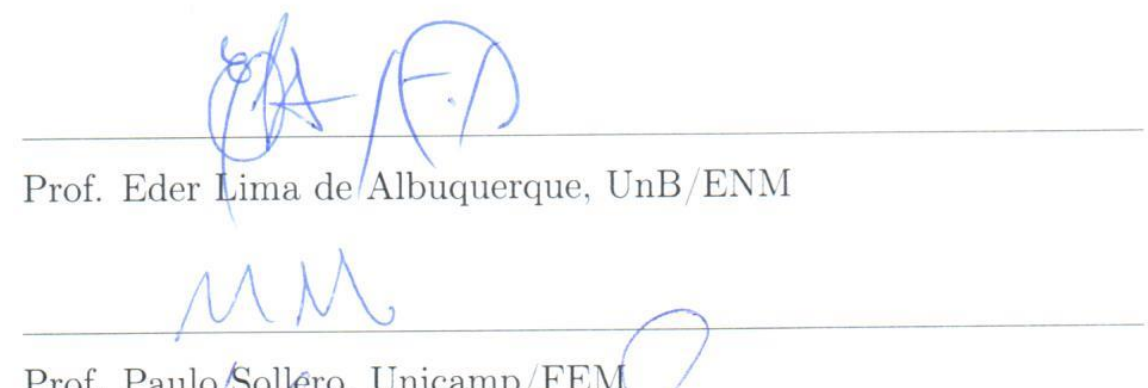

Prof. Paulo/Sollero, Unicamp/FEM

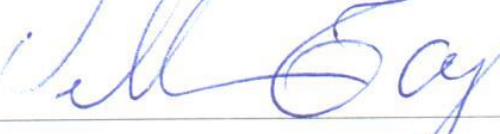

Prof. William Taylor Matias Silva, UNB/ENC

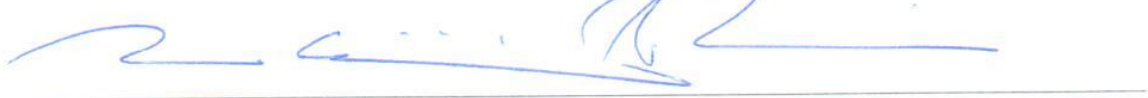

Prof. Marcus Vinicius Girão de Morais, UnB/ENM

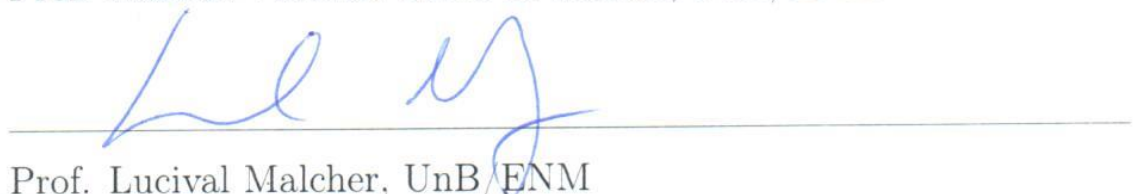

Prof. Lucival Malcher, UnB ENM 
1 Introdução 1

1.1 Métodos numéricos para a solução de equações diferenciais . . . . . . . 1

1.2 Método dos elementos de contorno . . . . . . . . . . . . . . . . . . . 2

1.3 Análise isogeométrica . . . . . . . . . . . . . . . . . . . 3

1.4 Objetivos, contribuição e desafios desse trabalho . . . . . . . . . . . . . 6

1.5 Descrição do trabalho . . . . . . . . . . . . . . . . . . . 6

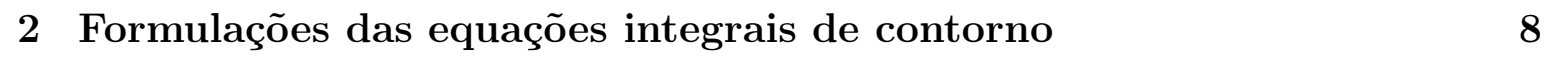

2.1 Introdução . . . . . . . . . . . . . . . . . . . . . . . . . . . . 8

2.2 Problemas potenciais $\ldots \ldots \ldots \ldots$

2.2 .1 Solução fundamental . . . . . . . . . . . . . . . . . . . 8

$2.2 .2 \quad$ Equação integral de contorno . . . . . . . . . . . . . . . . . 11

2.2 .3 Derivada da solução fundamental . . . . . . . . . . . . . . . . . 15

2.3 Problema elástico $\ldots \ldots \ldots \ldots$

2.3 .1 Teorema de Betti . . . . . . . . . . . . . . . . . . 16

2.3 .2 Equação integral de contorno . . . . . . . . . . . . . . 18

2.3 .3 Soluções fundamentais . . . . . . . . . . . . . . . . . . . . . . . . 19

2.4 Discretização . . . . . . . . . . . . . . . . . . . . . . . . . . . 23

3 Matrizes Hierárquicas e aproximação cruzada adaptativa 28

3.1 Introdução . . . . . . . . . . . . . . . . . . . . . . . . . . . . . . 28

3.2 Matrizes de baixo posto . . . . . . . . . . . . . . . . . . . 36

$3.2 .1 \quad$ Aproximação Cruzada . . . . . . . . . . . . . . . . . . 37

3.2 .2 Aproximação Cruzada Adaptativa . . . . . . . . . . . . . . . 38

3.2 .3 Aproximação Cruzada Adaptativa modificada . . . . . . . . . . 41

3.3 Clusterização hierárquica . . . . . . . . . . . . . . . . . . . . . . . . . . 42

3.4 Solução do sistema linear . . . . . . . . . . . . . . . . . . . . . . 45

3.4.1 O método dos resíduos mínimos generalizados (GMRES) . . . . 46

\begin{tabular}{|lll}
4 & Curvas e superfícies NURBS & 48
\end{tabular}

4.1 Introdução . . . . . . . . . . . . . . . . . . . . . . . . . . . . . . 48

4.2 Curvas de Bézier . . . . . . . . . . . . . . . . . . . . . . . . . . . . . . . . . . . 48

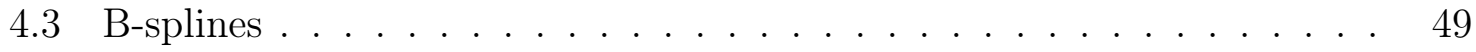

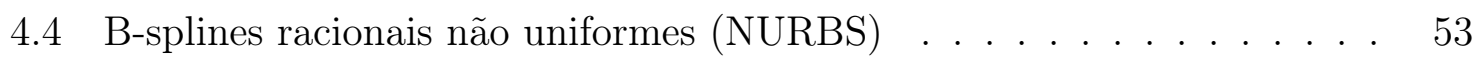

4.4 .1 Derivadas NURBS . . . . . . . . . . . . . . . . . . . . . . . . 54 
4.4 .2 Inserção de nós . . . . . . . . . . . . . . . . . . . . 55

4.4 .3 Decomposição de Bézier . . . . . . . . . . . . . . . . 56

4.5 Superfícies NURBS . . . . . . . . . . . . . . . . . . . . . . . 58

4.6 Superfícies NURBS aparadas . . . . . . . . . . . . . . . . . . . 60

5 Método dos elementos de contorno isogeométrico 62

5.1 Introdução . . . . . . . . . . . . . . . . . . . . 62

5.2 Formulação integral de contorno . . . . . . . . . . . . . . . . . . 62

5.3 Integrais fracamente singulares . . . . . . . . . . . . . . . . . 64

5.4 Integrais fortemente singulares . . . . . . . . . . . . . . . 65

5.5 Pontos de colocação $\ldots \ldots \ldots$. . . . . . . . . . . . . . . 67

5.6 Condição de contorno $\ldots \ldots \ldots$. . . . . . . . . . . . . . . . 68

5.7 Tensão no contorno $\ldots \ldots \ldots \ldots$. . . . . . . . . . . . . . 71

$\begin{array}{lll}6 & \text { Resultados numéricos } & 74\end{array}$

6.1 Problemas potencias . . . . . . . . . . . . . . . . . . 74

6.1 .1 Condução de calor em um cilindro . . . . . . . . . . . . . . . . . 74

6.1 .2 Placa retangular . . . . . . . . . . . . . . . . . 77

6.1 .3 Esfera . . . . . . . . . . . . . . . . . . . . . . 82

6.1 .4 Toroide . . . . . . . . . . . . . . . . . . 83

6.1 .5 Cilindro vazado . . . . . . . . . . . . . . . . . . . 84

6.1 .6 Cubo . . . . . . . . . . . . . . . . . . . 86

6.2 Problemas elásticos f . . . . . . . . . . . . . . . . . . . . . . . . . . . . 88

$6.2 .1 \quad$ Viga bidimensional . . . . . . . . . . . . . . . . . . . . . . . . 88

$6.2 .2 \quad$ Placa com furo central . . . . . . . . . . . . . . . . . . . . . . 89

$6.2 .3 \mathrm{Cubo} \ldots \ldots \ldots \ldots 9 . \ldots \ldots$

6.2 .4 Viga tridimensional . . . . . . . . . . . . . . . . . . 93

6.2 .5 Cilindro de parede espessa . . . . . . . . . . . . . . . 93

6.3 Análise do uso da aproximação cruzada adaptativa $\quad$. . . . . . . . . . . . 95

$6.3 .1 \quad$ Placa quadrada com furos . . . . . . . . . . . . . . . 95

6.3 .2 Cubo com furos . . . . . . . . . . . . . . . . . . . . . . . . . . 98

\begin{tabular}{lll}
\hline & Considerações finais & 100
\end{tabular}

7.1 Conclusão . . . . . . . . . . . . . . . . . . . . 100

7.2 Trabalhos futuros . . . . . . . . . . . . . . . . . . . . . . 101 


\section{Agradecimentos}

Ao professor Éder por, mais uma vez, tornar simples o que antes parecia impossível.

Ao professor Wrobel pelos inúmeros ensinamentos. 


\section{RESUMO}

Esta tese propõe formulações do método dos elementos de contorno isogeométricos acelerados pela aproximação cruzada adaptativa. As formulações são desenvolvidas para problemas potenciais e de elasticidade linear, bi e tridimensionais. Na formulação isogemétrica do método dos elementos de contorno, as funções de forma polinomiais são substituídas pelas funções splines racionais não-uniformes (sigla em inglês: NURBS). Uma vez que as NURBS são as funções usadas pelos programas de desenho assistidos por computador para representar as geometrias de figuras planas e sólidas, a discretização do modelo geométrico não é mais necessária. Contudo, por serem matematicamente mais complexas que as funções de forma polinomiais, o uso das NURBS aumenta muito o custo computacional da formulação. Ao se tratar as matrizes de influência do método dos elementos de contorno como matrizes hierárquicas e aproximá-las pelo método de aproximação cruzada adaptativa, o custo computacional é reduzido. Esta redução do custo é tão mais significativa quanto maior forem os tamanhos das matrizes. As formulações desenvolvidas são implementadas e aplicadas na análise de vários exemplos numéricos e seus resultados são comparados com o método dos elementos de contorno com o uso de funções de forma polinomiais.

A maior vantagem da formulação proposta é a diminuição do trabalho do engenheiro, uma vez que a etapa de geração da malha que, em problemas de larga escala, é o que demanda mais horas de trabalho é reduzido ou, na melhor das hipóteses, eliminado.

Palavras Chave: Elasticidade, Método dos elementos de contorno, ACA, isogeométrico. 


\begin{abstract}
This thesis proposes an isogeometric boundary element method accelerated by the adaptive cross approximation. The method is developed for potential and linear elastic formulations, in bi and tri-dimensional space. In the isogeometric method, the polynomial shape functions are substituted by the non-uniform rational B-splines (NURBS). Since NURBS are used by CAD software to model the geometry, the discretization of the geometric model is no longer necessary. However, since the NURBS functions are mathematically more complex than polynomials, the usage of such functions increases the computational cost of the method. By treating influence matrices of the boundary element method as hierarchical matrices and approximating them by the adaptive cross approximation, the computational cost is reduced. This reduction is more pronounced the bigger the sizes of the matrices. The developed method is implemented and applied in the analysis of different numerical examples and its results are compared to the boundary element method with polynomials as shape functions.

The biggest advantage of the proposed method is the decrease in the engineer's work, since the mesh generation step, that in large scale problems demands the most man-hours, is reduced or, in the best case scenario, eliminated.
\end{abstract}

Keywords: Elasticity, Boundary Element Method, ACA, isogeometric. 


\section{Lista de Figuras}

2.1 Definição do domínio . . . . . . . . . . . . . . . . . . . . . . . . . . . . 9

2.2 Contorno modificado. . . . . . . . . . . . . . . . . . . . . . . . . . . . . . . . 13

2.3 Alteração da geometria para consideração da singularidade no contorno 21

2.4 Descontinuidade da normal no contorno . . . . . . . . . . . . . . . 25

2.5 Descontinuidade da força de superfície . . . . . . . . . . . . . . . 25

$3.1 \quad$ Função $K$ para $\alpha=10^{0}$. . . . . . . . . . . . . . . . . . . . . . . . . . . 30

3.2 Função $K$ para $\alpha=10^{-1}$. . . . . . . . . . . . . . . . . . . . . . . 30

3.3 Função $K$ para $\alpha=10^{-2}$. . . . . . . . . . . . . . . . . . . . . . 31

3.4 Função $K$ para $\alpha=10^{-3}$. . . . . . . . . . . . . . . . . . . . . . 31

3.5 Erro na norma de Frobenius para a aproximação com SVD para $n=100.32$

3.6 Erro na norma de Frobenius para a aproximação com SVD para $n=200.32$

3.7 Erro na norma de Frobenius para a aproximação com SVD para $n=400.33$

3.8 Erro na norma de Frobenius para a aproximação com SVD para $n=800.33$

3.9 Divisão em blocos da matriz . . . . . . . . . . . . . . . . . . . . . . . . . . . . . 34

3.10 Redivisão em blocos da matriz . . . . . . . . . . . . . . . . . . . . . . . 35

3.11 Aproximação de baixo posto: algumas entradas da matriz original representam o bloco inteiro. . . . . . . . . . . . . . . . . 37

3.12 Configuração que causa problemas no ACA e a matriz resultante . . . . 41

3.13 Árvore binária . . . . . . . . . . . . . . . . . . . . . . . . . . . . . . . 43

$4.1 \quad$ Curva de Bézier $\ldots \ldots \ldots \ldots$

$4.2 \quad$ B-spline e seus segmentos para para $U_{1} \ldots \ldots \ldots \ldots$. . . . . . . . 51

$4.3 \quad$ Funções de base da B-spline para $U_{1} \ldots \ldots \ldots$. . . . . . . . . . . . . . 51

$4.4 \quad$ B-spline e seus segmentos para para $U_{2}$. . . . . . . . . . . . . . . . . . 52

$4.5 \quad$ Funções de base da B-spline para $U_{2} \ldots \ldots \ldots$. . . . . . . . . . . . . . . 52

4.6 NURBS em coordenadas homogêneas. . . . . . . . . . . . . . . . . 54

4.7 Sequência de uma curva e suas funções de base ao inserir repetidos nós 57

$4.8 \quad$ Falta de refinamento local nos produtos tensoriais. . . . . . . . . . . . . 59

$5.1 \quad$ Modificação das abcissas de Greville. . . . . . . . . . . . . . . . . . . . 69

5.2 Sistema de coordenadas tangenciais bidimensionais. . . . . . . . . . . . 71

5.3 Sistema de coordenadas tangenciais tridimensionais. . . . . . . . . . . 73

$6.1 \quad$ Problema potencial simples em uma região anelar . . . . . . . . . . . . 75

6.2 Resultado do fluxo no ponto A . . . . . . . . . . . . . . . 75 
6.3 Resultado da temperatura no ponto B $\ldots \ldots \ldots$. . . . . . . 76

6.4 Erros obtidos ao longo de $\mathrm{AB}$. . . . . . . . . . . . . . . . . 76

6.5 Placa retangular. . . . . . . . . . . . . . . . . . . . . . . . . . . . . . . . . . . . 77

6.6 Temperatura obtida para a placa retangular . . . . . . . . . . . . . 78

6.7 Convergência para a solução analítica do ponto E - método proposto . $\quad 79$

6.8 Convergência para a solução analítica do ponto E - sem a utilização do método proposto . . . . . . . . . . . . . . . 80

6.9 Tempo gasto na montagem da matriz em relação ao número de nós . . 80

6.10 Tempo gasto na montagem da matriz em relação a ordem da NURBS . $\quad 81$

6.11 Esfera discretizada pelas NURBS . . . . . . . . . . . . . . . . . . 82

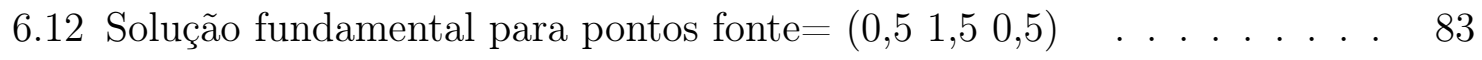

6.13 Solução fundamental para distintos pontos fonte $=\left(\begin{array}{lll}1,5 & 0,5 & 0,5\end{array}\right)$. . . 84

6.14 Toroide discretizado pelas NURBS . . . . . . . . . . . . . . . . . . 85

6.15 Cilindro discretizado pelas NURBS . . . . . . . . . . . . . . . . . . 85

6.16 Cubo discretizado pelas NURBS . . . . . . . . . . . . . . . . . . . 87

6.17 Diferentes configurações de viga . . . . . . . . . . . . . . . . . . 88

6.18 Problema da placa com furo . . . . . . . . . . . . . . . . . . . . . 92

6.19 Cubo tracionado . . . . . . . . . . . . . . . . . . . . . . . . . . 93

6.20 Viga tridimensional com carregamento distribuído . . . . . . . . . . . . 94

6.21 Deflexão máxima de uma viga de razão 10:1 . . . . . . . . . . . . . . . 94

6.22 Cilindro de parede espessa com pressão hidrostática . . . . . . . . . . . 95

6.23 Placa com furos . . . . . . . . . . . . . . . . . . . . . 96

6.24 Condutividade térmica equivalente para quantidades distintas de furos. $\quad 97$

6.25 Tempo de execução do programa para a placa com distintas quantidades de furos. . . . . . . . . . . . . . . . . . . . . . . . . . . . . 97

6.26 Tempo de execução do programa para a placa com distintos valores de $\eta$. 98

6.27 Cubo com 27 furos sob tração. . . . . . . . . . . . . . . . . . . . . . . . 99 


\section{Lista de Tabelas}

3.1 Memória gasta na aproximação com SVD. . . . . . . . . . . . . . . . . 34

$3.2 \quad$ Valores de $m$ para as diferentes áreas da figura $|3.9| \ldots$. . . . . . . . . 35

3.3 Memória gasta na aproximação com a divisão em blocos da figura 3.9. 35

3.4 Valores de $m$ para as diferentes áreas da figura 3.10 . . . . . . . . . . . . 36

3.5 Memória gasta na aproximação com a divisão em blocos da figura 3.10 ] 36

$6.1 \quad$ Erro RMS normalizado para o exemplo da esfera . . . . . . . . . . . . . 83

6.2 Erro RMS normalizado para o exemplo do toroide . . . . . . . . . . . . 84

$6.3 \quad$ Erro RMS normalizado para o exemplo do cilindro . . . . . . . . . . . . 86

6.4 Erro RMS normalizado para o exemplo do cubo . . . . . . . . . . . . . 87

6.5 Viga de razão $4: 1 \ldots \ldots$. . . . . . . . . . . . . . . . . . . . 90

$6.6 \quad$ Viga de razão $10: 1 \ldots \ldots$. . . . . . . . . . . . . . . . . . . . . . . . 91

6.7 Concentradores de tensão para uma placa com furo central . . . . . . . $\quad 92$

6.8 Tensão circunferencial para um cilindro de parede espessa . . . . . . . . 95

6.9 Modulo de elasticidade equivalente para um cubo com 27 furos . . . . . 99 


\section{Lista de Símbolos}

\section{Letras gregas}

$\Gamma-$ contorno

$\xi$ - Coordenada adimensional

$\varepsilon$ - tensor deformação, erro

$\Delta$ - Delta de Dirac

$\Omega$ - dominio

$\sigma-$ tensor tensão

\section{Letras arábicas}

$b$ - Força de campo

$B$ - Pontos de controle

d-Grau da curva

$E$ - Matriz de transformação

$G_{i}-$ Vetor de Galerkin

$J$ - Jacobiano

$J$ - Base de Bernstein

$k$ - Condutividade térmica

ln - Logarítimo natural

$m$ - Número de nós

$n$ - Normal, ordem

$N$ - Funções de base

$p$ - ponto no domínio

$q$ - Quantidade de calor

$Q$ - ponto no contorno

$r$ - Raio

$R$ - Funções de base racional

$T$ - Temperatura

$t$ - Forças de superfície

$T_{i j}$ - solução fundamental de tração

$u$ - Potencial, deslocamento

$U$ - Vetor de nós

$U_{i j}$ - solução fundamental de deslocamento 


\section{Lista de Abreviações}

ACA - Aproximação Cruzada Adaptativa

bicgstab - gradiente conjugado bi-estabilizado

CAD - Desenho Assistido por Computador

GMRES - resíduo mínimo generalizado

MDF - Método das Diferenças Finitas

MEC - Método dos Elementos de Contorno

MEF - Método dos Elementos Finitos

MIN-RES - Resíduo Mínimo

NURBS - B-spline não-uniforme racional

PCA - Análise dos Componentes Principais

SVD - Decomposição de valor singular 


\section{INTRODUÇÃO}

\subsection{Métodos numéricos para a solução de equações diferenciais}

Quase todos os processos físicos da natureza podem ser descritos por uma equação diferencial, usando as leis fundamentais da física como o equilíbrio, a preservação de energia e outros. Isso, combinado com as leis descrevendo o comportamento dos materiais, faz com que os modelos matemáticos sejam extremamente precisos, ou seja, tenham um grande poder de reproduzir os fenômenos naturais. Estas previsões reduzem significativamente o custo no desenvolvimento de novos produtos pois reduzem a demanda por ensaios experimentais que, por sua vez, possuem custos elevados se comparado com a análise teórica. Entretanto, apenas uma classe limitada de problemas tem uma solução analítica. Sem técnicas numéricas seria praticamente impossível resolver problemas práticos de engenharia com um grau de precisão razoável. A maior parte das técnicas numéricas se baseiam no princípio que é possível obter equações e relações que descrevem o comportamento de uma pequena parte diferencial do corpo, construindo modelos discretos a partir do modelo contínuo. Com a evolução dos computadores, os métodos numéricos têm-se tornado cada vez mais precisos, robustos e acessíveis. O grande avanço na velocidade de processamento e na capacidade de armazenamento dos computadores fez com que o tamanho dos problemas analisados pudessem crescer de maneira substancial. Ao mesmo tempo, os algorítimos e métodos numéricos, também evoluiram. Novas estratégias de resolução de sistemas lineares e representação de matrizes reduziram a complexidade de muitas formulações, em especial das do método dos elementos de contorno como ficará mostrado nesse trabalho.

Depois de décadas de desenvolvimento, o método dos elementos de contorno (MEC) se firmou entre os métodos numéricos para solução de equações diferenciais parciais. Comparando com métodos mais populares, como o método dos elementos finitos (MEF) e o método das diferenças finitas (MDF), que são classificados como métodos de domínio, o MEC se diferencia por ser um método de contorno, significando que a discretização numérica é conduzida em uma dimensão espacial reduzida, por exemplo, para problemas de três dimensões, a discretização é realizada somente nas superfícies de contorno. Essa redução leva a um sistema com menos graus de liberdade. O efeito é ainda mais pronunciado quando o domínio é infinito. Domínios infinitos tem de ser truncados ou aproximados em métodos de domínio. No entanto, o MEC automaticamente modela o comportamento no infinito sem a necessidade de uma malha para aproximá-la.

Atualmente fazer a malha, considerando o usuário, é a parte mais trabalhosa e cara 
no processo de simulação, particularmente no MEF (BELYTSCHKO et al., 1994). Sem a necessidade de lidar com a malha no interior do domínio, o MEC é mais eficiente na geração de malha. Para problemas de fronteira móvel, o ajuste da malha é muito mais simples com o MEC. A análise isogeométrica é uma tentativa de facilitar a geração da geometria usando a discretização dos softwares de desenho assistido por computador (CAD), onde o MEC tem significativas vantagens que serão mostradas nas sessões a seguir.

\subsection{Método dos elementos de contorno}

Apesar das principais propriedades das equações diferenciais já serem bem estabelecidas no século 19, somente no trabalho de Fredholm (1903) as primeiras investigações foram feitas nas equações integrais. Ele apresentou as condições de existência e unicidade, conhecidas como os teoremas de Fredholm. Desde então, as equações integrais foram estudadas intensamente.

O MEC é um método numérico que resolve equações diferencias parciais que foram transformadas em equações integrais. Ele pode ser aplicado em diversas áreas da ciência e engenharia como mecânica dos fluídos, acústica, electromagnetismo, mecânica da fratura, plasticidade, estabilidade, elasticidade e outros.

Uma das principais diferenças entre o MEC e o MEF se refere as funções peso usadas para encontrar a forma fraca das equações diferenciais parciais. O MEF usa funções arbitrárias e simples. Enquanto o MEC usa soluções analíticas representando os efeitos de uma carga pontual em outro ponto de um domínio infinito (BECKER, 1992), soluções essas conhecidas como soluções fundamentais. O uso dessas funções são a razão da maior precisão do MEC em relação ou MEF, particularmente em regiões onde as variáveis apresentam alto gradiente.

Atualmente, diversos livros que tratam sobre o MEC, em diversas áreas de aplica-

ção, podem ser encontrados, como por exemplo em Wrobel (2002), Brebbia, Wrobel e Telles (1984), Wrobel e Aliabadi (2002), Becker (1992), Banerjee e Butterfield (1981), Katsikadelis (2002), Katsikadelis (2016), Beer (2000), Beer (2015), Brebbia e Walker (2013), Brebbia e Dominguez (1996) entre muitos outros.

A ideia que o MEC é superior aos métodos de domínio uma vez que eles reduzem a dimensão do problema ao discretizar apenas sua superfície é um tanto quanto simplista. As matrizes geradas pelo método são cheias, não-simétricas e tem um custo alto de montagem. Várias técnicas tem sido investigadas na tentativa de superar essas dificuldades: wavelets (BUCHER et al., 2002), solver baseado em blocos (RIGBY; ALIABADI, 1995: CROTTY, 1982), processos de aglutinação (KANE; KUMAR; KASHAVA, 1990) e técnicas iterativas (MANSUR; ARAUJO; MALAGHINI, 1992; BARRA et al., 1992). 
Uma técnica popular é o método dos multipolos (AOKI et al., 2004; CARRIER; GREENGARD; ROKHLIN, 1988; CHENG; GREENGARD; ROKHLIN, 1999; CHEW et al., 2004; GREENGARD; ROKHLIN, 1987; HE; LIM; LIM, 2008; ROKHLIN, 1985, WANG; YAO, 2005; LIU, 2009). O método dos multipolos, apesar de tornar o MEC mais eficiente para problemas de larga escala, precisa conhecer previamente a expansão da solução fundamental em séries. Todos os termos da série, para uma determinada precisão, devem ser calculados anteriormente e depois integrados, o que altera significativamente o processo de integração do MEC padrão. Outra técnica muito popular é baseada na aproximação cruzada adaptativa (ACA) (HACKBUSCH, 2016, BEBENDORF; GRZHIBOVSKIS, 2006, BEBENDORF; RJASANOW, 2003, BEBENDORF, 2000). Essa técnica reduz eficientemente o tempo de montagem e o custo de armazenamento das matrizes do MEC.

A ACA aproxima as matrizes densas do MEC por matrizes hierárquicas, que possibilita uma representação com menor quantidade de dados. Essa representação se baseia em uma estrutura de árvore que descreve o particionamento em blocos da matriz. Cada bloco contém uma aproximação de baixo posto ou a própria matriz exata. São nesses blocos aproximados que estão os ganhos computacionais. Hackbusch (1999), Hackbusch (2000), Grasedyck e Hackbusch (2003) definem a aritmética de matrizes hierárquicas incluindo operações como adição, multiplicação, inversão e fatoração LU utilizando as vantagens obtidas pelas aproximações de baixo posto.

Para resolver um sistema de equações lineares, com muitos graus de liberdade, convém aplicar-se métodos iterativos (GREENBAUM, 1997) a fim de se obter uma performance superior. Qualquer um dos métodos clássicos, como Jacobi, Gauss-Seidel, resíduo mínimo (MIN-RES), gradiente conjugado, gradiente conjugado bi-estabilizado (bicgstab) ou resíduo mínimo generalizado (GMRES), tem como principal custo a multiplicação matriz-vetor que é bem mais eficiente quando se trata de matrizes hierárquicas. Um código MEC que utiliza ACA, matrizes hierárquicas e técnicas iterativas se torna competitivo e pode acelerar em muito o tempo necessário requerido pelas simulações.

\subsection{Análise isogeométrica}

A tecnologia predominante usada pelo CAD na representação de geometrias complexas é a B-spline não-uniforme racional (NURBS). Isso possibilita reproduzir de maneira exata entidades que seriam apenas aproximadas por funções polinomiais, incluindo seções cônicas circulares. Existe uma vasta literatura que trata dos diferentes aspectos das NURBS (por exemplo: Peigl e Tiller (1996), Rogers (2000)) e as décadas de pesquisa resultaram em diversos algorítimos eficientes para a avaliação rápida e 
refinamento. O conceito principal apresentado em Hughes, Cottrell e Bazilevs (2005) é empregar as NURBS não só como discretização da geometria, mas também como ferramenta de discretização na análise, atribuindo a denominação de análise isogeométrica para essa família de métodos. Posteriormente, o livro de Cottrell, Hughes e Bazilevs (2009) foi publicado, dedicando-se exclusivamente a análise isogeométrica utilizando o método dos elementos finitos (MEF). Esse tipo de análise já foi aplicado nas mais diversas áreas, incluindo mecânica dos sólidos, mecânica dos fluídos e contato mecânico. A ideia de usar tecnologias do CAD no MEF já vem sendo discutida desde Kagan et al. (2000) onde B-splines são usadas como as funções de forma no MEF. No MEC, uma formulação utilizando B-splines é apresentada inicialmente em Cabral et al. (1990) e Cabral, Wrobel e Brebbia (1991) mas ainda sem ter a preocupação na integração ao CAD. Essa integração foi observada, posteriormente, em Simpson et al. (2012) e Simpson et al. (2013).

As NURBS se mostraram vantajosas na formulação de contato, no MEF, quando comparada a discretização tradicional. A presença de superfícies facetadas pode gerar saltos e oscilações nas respostas de forças de superfície, ao menos que malhas muito finas sejam utilizadas. As superfícies suaves geradas pelas NURBS geram, consequentemente, melhores resultados para as tensões de contato. Esse problema pode ser observado em Temizer, Wriggers e Hughes (2011), Temizer, Wrigger e Hughes (2012) $\mathrm{e} \mathrm{Lu}(2011)$.

Problemas de placas e cascas são outro campo onde a suavidade das funções de base das NURBS são vantajosas, particularmente para placas finas, onde formulações sem rotação podem ser facilmente construídas (KIENDL et al., 2010, BENSON et al. 2013, DENG et al., 2015, KIENDL; BLETZINGER; LINHARD J. WÜCHNER, 2009). Observa-se ainda um efeito reduzido do shear-locking quando comparado com os elementos padrões. Em Lu (2009) e Lu, Zhou e Xianlian (2011), novos elementos suaves e curvos são propostos com sucesso, utilizando os conceitos isogeométricos.

Na mecânica dos fluidos e na análise de interação fluido-estrutura, em Nielsen et al. (2011), Bazilevs e Akkerman (2010) e Bazilevs et al. (2008), as NURBS foram utilizadas com sucesso. A suavidade e alta continuidade das curvas as tornaram especialmente interessantes nesse casos. Ao utilizar as NURBS, equações que contém derivadas de alta ordem das variáveis de campo podem ser criadas de maneira direta. Exemplos desse caso são a equação de Cahn-Hilliard (GÓMEZ et al., 2008), modelos de dano com gradiente (VERHOOSEL et al., 2011b), elasticidade gradiente (FISCHER et al., 2011) e a equação de Kohn-Sham para modelagem da estrutura eletrônica de materiais semi-condutores (MASUD; KANNAN, 2012).

O refinamento $\mathrm{k}$ (exclusivo da análise isogeométrica), que consiste numa elevação 
de ordem e inclusão de nós simultânea (HUGHES; COTTRELL; BAZILEVS, 2005), se mostrou mais robusto e preciso que o típico refinamento $\mathrm{p}$ em problemas de vibração estrutural (COTTRELL et al., 2006; HUGHES; REALI; SANGALLI, 2008; THAI et al., 2012).

O conceito isogeométrico também está presente nos métodos sem malha, criando métodos baseados em splines, como apresentado em Wang e Zhang (2014), Shaw e Roy (2008), Kim e Youn (2012).

Apesar de aplicadas com êxito em diversas áreas do MEF, existem algumas desvantagens inerentes as NURBS de um ponto de vista geométrico. A impossibilidade de garantir que diferentes superfícies curvas se mantenham seladas é um dos maiores desafios presentes na geometria computacional. Além disso, a estrutura de produto tensorial presente nas superfícies das NURBS impossibilita o refinamento local (HUGHES; COTTRELL; BAZILEVS, 2005).

A alternativa mais promissora é usar as T-splines, apresentadas em Sederberg et al. (2003), que tem as mesmas funções de base das NURBS mas tem uma diferente estrutura de nós. As T-splines solucionam o problemas de vazios entre superfícies distintas e permite o refinamento local. Entretanto, em Buffa, Cho e Sangalli (2010) verifica-se a existência de malhas inadequadas para análise, sendo necessário cuidados especiais na criação delas (MORGENSTERN; PETERSEIM, 2014).

Apesar da extensa utilização das T-splines, como por exemplo em Bazilevs et al. (2010), Thomas e Scott (2015), Dörfel, Jüttler e Simeon (2010), Evans et al. (2015), Thomas et al. (2015), Scott et al. (2013), elas ainda não foram adotadas na grande maioria dos programas de CAD e alternativas continuam sendo propostas como polycube splines (WANG et al., 2008; LI et al., 2013), PHT-splines (DENG et al., 2008) e LR-splines (DOKKEN; LYCHE; PETTERSEN, 2013). Mesmo sendo possível realizar refinamento adaptativo local nas T-splines, a adição de nós nesse contexto tem alta complexidade enquanto é relativamente simples realizar essa operação em PHT-splines.

Outras estratégias incluem o uso de B-splines hierárquicas BORNEMANN; CIRAK, 2013, VUONG, 2012, SCHILLINGER et al., 2012) e de splines Powel-Sabin (VANRAES, 2004; SPELEERS et al., 2012). As B-splines hierárquicas tornam possível o refinamento local por meio da propriedade das funções de base hierárquicas que podem ser reescritas por uma combinação linear delas mesmas. As Powel-Sabin splines descrevem superfícies numa triangulação, diferindo do usual produto tensorial das B-splines, o que facilita a discretização de topologias complexas. Nelas, usualmente, múltiplas parametrizações das NURBS são necessárias. Garantir certa continuidade ao fazer essa união não é tarefa trivial. Superfícies aparadas são apresentadas em Beer, Marussig e Zechner (2015), Kim, Seo e Youn (2010), Cárdenas e Rubén (2009), Se- 
villa, Fernández-Méndez e Huerta (2011), Schmidt, Wüchner e Bletzinger (2012) como alternativa na representação desses domínios complexos.

Apesar de mais desenvolvida no contexto do MEF, a análise isogeométrica é realmente isogeométrica somente no MEC. Nos modelos CAD somente as superfícies são definidas, o interior dos sólidos não é explicitamente modelado. No MEF, essa definição é necessária, o que exige uma etapa adicional de análise onde as representações CAD tem que ser convertidas para modelos sólidos. Enquanto isso, no MEC, somente a discretização do contorno, que é completamente definida pelo CAD, é necessária.

Além disso, a alta continuidade das funções de base das NURBS diminui a esparsidade das matrizes no MEF, aumentando consideravelmente o custo computacional do método (COLLIER et al., 2012). Esse efeito não é observado no MEC, já que as matrizes geradas são cheias. Outra desvantagem do MEF isogeométrico é que a quadratura de Gauss deixa de ser ótima uma vez que o integrando deixa de ser um simples polinômio. Esse comportamento já é observado mesmo no MEC padrão já que a solução fundamental, que faz parte do integrando, não é um polinômio.

\subsection{Objetivos, contribuição e desafios desse trabalho}

O presente trabalho tem como objetivo o desenvolvimento e implementação de uma formulação isogeométrica juntamente com elementos de contorno rápido, utilizando a ACA como método rápido e as NURBS como funções de base isogeométrica. Não foi encontrado na literatura nenhum trabalho onde a formulação isogeométrica do MEC é usada em conjunto com métodos rápidos. Um dos maiores desafios quando estas formulações são usadas em conjunto é a aplicação das condições de contorno, como ficará claro ao longo desse trabalho.

A formulação proposta lida com essas questões de maneira satisfatória, resultando em um método acelerado que não necessita geração de malha por obter todas as entidades diretamente da geometria.

\subsection{Descrição do trabalho}

Neste trabalho são apresentadas formulações do método dos elementos de contorno isogeométrico para análise problemas potenciais e elásticos aceleradas pela ACA. No próximo capítulo, o capítulo 2 , a teoria clássica do método dos elementos de contorno para problemas elásticos e potenciais é apresentada. As soluções fundamentais bi e tri-dimensionais para as duas formulações são introduzidas. A equação integral de contorno é obtida e discretizada usando funções polinomiais de qualquer ordem. $\mathrm{O}$ capítulo 3 apresenta a formulação básica da ACA usando o formato de matriz hierár- 
quica em conjunto com método iterativo na solução do sistema linear. O procedimento e todos os aspectos matemáticos da implementação são descritos. No capítulo 4 são apresentadas as técnicas isogeométricas, detalhando a formulação das NURBS. No capítulo 5, as NURBS são introduzidas como funções de forma na formulação do método dos elementos de contorno, dando origem à formulação do método dos elementos de contorno isogeométricos acelerado pela ACA. Uma atenção especial é dada para a integração, colocação e na implementação das condições de contorno. O capítulo 6 contém os resultados numéricos obtidos. No sétimo e último capítulo estão localizadas as conclusões e sugestões para futuros trabalhos. 


\section{FORMULAÇÕES DAS EQUAÇÕES INTEGRAIS DE CONTORNO}

\subsection{Introdução}

A base do MEC são atribuídas às formulações clássicas do século XIX de Somigliana (1885), para problemas elásticos, e de Fredholm (1903), para problemas potencias. E, mais tarde, foram continuadas por Mikhlin et al. (1964) e Neuber (1954). Em 1963, foram apresentados os primeiros resultados na solução de problemas bidimensionais pelo MEC por Jaswon (1963), Symm (1963). E, em Rizzo (1967), apresentou-se o MEC para problemas de elasticidade bidimensional, que foi estendido para problemas tridimensionais em Cruse (1969). A introdução de elementos de maior ordem em Lachat e Watson (1976) aumentou a eficácia do método. As seções seguintes mostram o desenvolvimento matemático do método. Mais detalhes podem ser encontrados em diversos livros, como Wrobel e Aliabadi (2002), Kane (1994), Brebbia (1982), Beer (2015), Katsikadelis (2016).

\subsection{Problemas potenciais}

Nessa seção, o MEC será desenvolvido para problemas que são descritos pela equação potencial:

$$
\nabla^{2} u=f
$$

Essa é a equação governante da teoria potencial. Para $f=0$, a equação 2.1 é conhecida como equação de Laplace. Enquanto para $f \neq 0$, essa equação é conhecida como equação de Poisson. Diversos são os problemas descritos por essas equações, por exemplo, torção de barras não circulares, deflexão de membranas elásticas, flexão de placas apoiadas, transferência de calor, escoamento de fluídos ideais, escoamento em meios porosos, difusão de íons, fluxo elétrico, entre outros.

Na figura 2.1 pode-se observar um domínio $\Omega$ com seu contorno $\Gamma$ divido em duas partes $\Gamma_{1}$ e $\Gamma_{2}$, onde $\Gamma_{1}$ corresponde às condições de contorno de Dirichlet (potencial conhecido) e $\Gamma_{2}$ às condições de contorno de Neumann (derivada do potencial conhecida).

\subsubsection{Solução fundamental}

A solução fundamental, que é a base da formulação do MEC, para a equação de Laplace, corresponde à resposta do potencial em um meio infinito quando a fonte é 


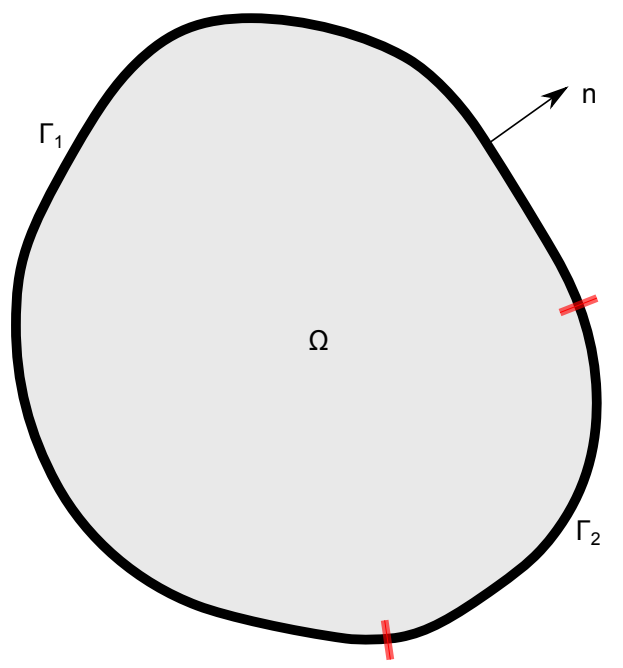

Figura 2.1: Definição do domínio

concentrada em um ponto. Matematicamente, ela corresponde à solução particular analítica da equação de Fourier quando o termo não homogêneo (termo referente à fonte) é igual ao delta de Dirac.

$$
\nabla^{2} u^{*}=\Delta(P, Q)
$$

A função do delta de Dirac, descrita como:

$$
\Delta(X, x)=\left\{\begin{array}{l}
\infty \text { se } x=X \\
0 \text { no restante do intervalo }
\end{array},\right.
$$

ou seja, a função delta de Dirac é zero em todos os pontos do domínio com exceção do ponto $x=X$ onde ela se fica igual a infinito.

A função delta de Dirac tem outras propriedades interessantes:

$$
\begin{gathered}
\int_{-\infty}^{\infty} \Delta(X, x) d x=1 \\
\int_{a}^{b} \Delta(X, x) f(x) d x=f(X) \text { para } a<X<b
\end{gathered}
$$

Uma solução particular da equação 2.2 é chamada de solução fundamental da equação (2.1). Ela pode ser obtida escrevendo a equação 2.2 com a origem no ponto fonte em coordenadas polares, para o caso 2D:

$$
\frac{1}{r} \frac{\partial}{\partial r}\left(r \frac{\partial u^{*}}{\partial r}\right)+\frac{1}{r^{2}} \frac{\partial^{2} u^{*}}{\partial \theta^{2}}=\Delta(P, Q)
$$


e em coordenadas esféricas para o caso 3D:

$$
\frac{1}{r^{2}} \frac{\partial}{\partial r}\left(r^{2} \frac{\partial u^{*}}{\partial r}\right)+\frac{1}{r^{2} \sin \theta} \frac{\partial}{\partial \theta}\left(\sin \theta \frac{\partial u^{*}}{\partial \theta}\right)+\frac{1}{r^{2} \sin ^{2} \theta} \frac{\partial^{2} u^{*}}{\partial \varphi^{2}}=\Delta(P, Q)
$$

em que $r$ é a distância entre o ponto onde a fonte é aplicada (ponto fonte $P$ ) e o ponto onde o potencial é medido (ponto campo $Q$ ). Considerando que a solução é axissimétrica e que o lado direito é zero para todos os pontos menos a origem, a equação 2.6 pode ser escrita como:

$$
\frac{1}{r} \frac{\partial}{\partial r}\left(r \frac{\partial u^{*}}{\partial r}\right)=0
$$

e a equação (2.7) como:

$$
\frac{1}{r^{2}} \frac{\partial}{\partial r}\left(r^{2} \frac{\partial u^{*}}{\partial r}\right)=0
$$

Depois de ser integrada duas vezes gera no caso bidimensional:

$$
u^{*}=A \log (r)+B
$$

e no caso tridimensional:

$$
u^{*}=\frac{A}{r}+B
$$

onde $A$ e $B$ são constantes arbitrárias. Como procura-se uma solução particular, podese definir $B=0$. A constante $A$ pode ser determinada aplicando as equações em um domínio circular tendo o ponto fonte como origem junto com a identidade de Green, gerando:

$$
\int_{\Omega} \nabla^{2} u^{*} d \Omega=\int_{\Gamma} \frac{\partial u^{*}}{\partial n} d s .
$$

Como num círculo $\frac{\partial u^{*}}{\partial n}=\frac{\partial u^{*}}{\partial r}$, a equação pode ser escrita como:

$$
\int_{\Omega} \Delta(P, Q) \cdot d \Omega=\int_{0}^{2 \pi} A \frac{1}{r} r d \theta .
$$

Ao integrar o lado direito e aplicar as propriedades do delta de Dirac no lado esquerdo obtém-se:

$$
1=2 \pi A,
$$

portanto:

$$
A=\frac{1}{2 \pi} .
$$


A solução fundamental bidimensional se torna, finalmente:

$$
u^{*}=\frac{\log (r)}{2 \pi}
$$

Fazendo o mesmo procedimento em um domínio esférico, obtém-se

$$
\int_{\Omega} \Delta(P, Q) \cdot d \Omega=\int_{0}^{2 \pi} \int_{0}^{\pi} A \frac{1}{r^{2}} r^{2} \sin \theta d \theta d \varphi .
$$

Ao integrar o lado direito duas vezes e aplicar as propriedades do delta de Dirac no lado esquerdo obtém-se:

$$
1=4 \pi A
$$

portanto

$$
A=\frac{1}{4 \pi}
$$

A solução fundamental tridimensional se torna, finalmente:

$$
u^{*}=\frac{1}{4 \pi r}
$$

\subsubsection{Equação integral de contorno}

Dada a equação de Laplace:

$$
\nabla^{2} u=0
$$

multiplicando a equação 2.21 por uma função peso $\omega(x, y)$ e integrando sobre o domínio $A$, assume-se que o resultado da integral é zero (método dos resíduos ponderados). Assim, tem-se:

$$
\begin{aligned}
\iint_{A}\left(\nabla^{2} u\right) \omega d A & =0, \\
\iint_{A}\left(\frac{\partial^{2} u}{\partial x^{2}}+\frac{\partial^{2} u}{\partial y^{2}}\right) \omega d A & =0, \\
\iint_{A} \frac{\partial^{2} u}{\partial x^{2}} \omega d A+\iint_{A} \frac{\partial^{2} u}{\partial y^{2}} \omega d A & =0 .
\end{aligned}
$$

Pelo teorema de Gauss-Green, tem-se:

$$
\int_{s} f(x, y) n_{x} d s=\int_{A} \frac{\partial f}{\partial x} d A
$$

onde $f$ é uma função qualquer, $n_{x}$ é a componente na direção $x$ do vetor $\mathbf{n}$ normal ao contorno $s$ da área $A$. Aplicando o teorema dado na primeira parcela da Eq.2.22, 
tem-se:

$$
\int_{s} \frac{\partial u}{\partial x} \omega n_{x} d s=\int_{A} \frac{\partial}{\partial x}\left(\frac{\partial u}{\partial x} \omega\right) d A
$$

Aplicando a regra da derivada do produto de funções, tem-se:

$$
\int_{s} \frac{\partial u}{\partial x} \omega n_{x} d s=\int_{A} \frac{\partial^{2} u}{\partial^{2} x} \omega d A+\int_{A} \frac{\partial u}{\partial x} \frac{\partial \omega}{\partial x} d A
$$

Escrevendo os termos da equação de forma conveniente, segue:

$$
\int_{A} \frac{\partial^{2} u}{\partial x^{2}} \omega d A=\int_{s} \frac{\partial u}{\partial x} \omega n_{x} d s-\int_{A} \frac{\partial u}{\partial x} \frac{\partial \omega}{\partial x} d A .
$$

De modo análogo, obtém-se:

$$
\int_{A} \frac{\partial^{2} u}{\partial y^{2}} \omega d A=\int_{s} \frac{\partial u}{\partial y} \omega n_{y} d s-\int_{A} \frac{\partial u}{\partial y} \frac{\partial \omega}{\partial y} d A
$$

Substituindo as Eqs. 2.26) e 2.27) na Eq.(2.22, tem-se:

$$
\int_{s}\left(\frac{\partial u}{\partial x} \omega n_{x}+\frac{\partial u}{\partial y} \omega n_{y}\right) d s-\int_{A}\left(\frac{\partial u}{\partial x} \frac{\partial \omega}{\partial x}+\frac{\partial u}{\partial y} \frac{\partial \omega}{\partial y}\right) d A=0
$$

Simplificando

$$
\int_{s} \frac{\partial u}{\partial n} \omega d s-\int_{A}\left(\frac{\partial u}{\partial x} \frac{\partial \omega}{\partial x}+\frac{\partial u}{\partial y} \frac{\partial \omega}{\partial y}\right) d A=0 .
$$

Aplicando o teorema de Gauss-Green e usando a regra da derivado do produto de funções pode-se obter as seguintes igualdades:

$$
\int_{A} \frac{\partial u}{\partial x} \frac{\partial \omega}{\partial x} d A=\int_{s} u \frac{\partial \omega}{\partial x} n_{x} d s-\int_{A} u \frac{\partial^{2} \omega}{\partial x^{2}} d A
$$

e

$$
\int_{A} \frac{\partial u}{\partial y} \frac{\partial \omega}{\partial y} d A=\int_{s} u \frac{\partial \omega}{\partial y} n_{y} d s-\int_{A} u \frac{\partial^{2} \omega}{\partial y^{2}} n_{y} d A .
$$

Substituindo as equações 2.30) e 2.31) na equação 2.29), tem-se:

$$
\begin{gathered}
\int_{s} \frac{\partial u}{\partial n} \omega d s-\int_{s}\left(u \frac{\partial \omega}{\partial x} n_{x}+u \frac{\partial \omega}{\partial y} n_{y}\right) d s+\int_{A} u\left(\frac{\partial^{2} \omega}{\partial x^{2}}+\frac{\partial^{2} \omega}{\partial y^{2}}\right) d A=0, \\
\int_{s} \frac{\partial u}{\partial n} \omega d s-\int_{s} u \frac{\partial \omega}{\partial n} d s+\int_{A} u \nabla^{2} \omega d A=0 .
\end{gathered}
$$

Com o objetivo de obter uma equação integral que não possua integrais de domínio, a função $\omega$ deve ser escolhida de forma que a integral de domínio da equação 2.32 desapareça. Qualquer função harmônica, ou seja, função cujo o laplaciano é igual a 


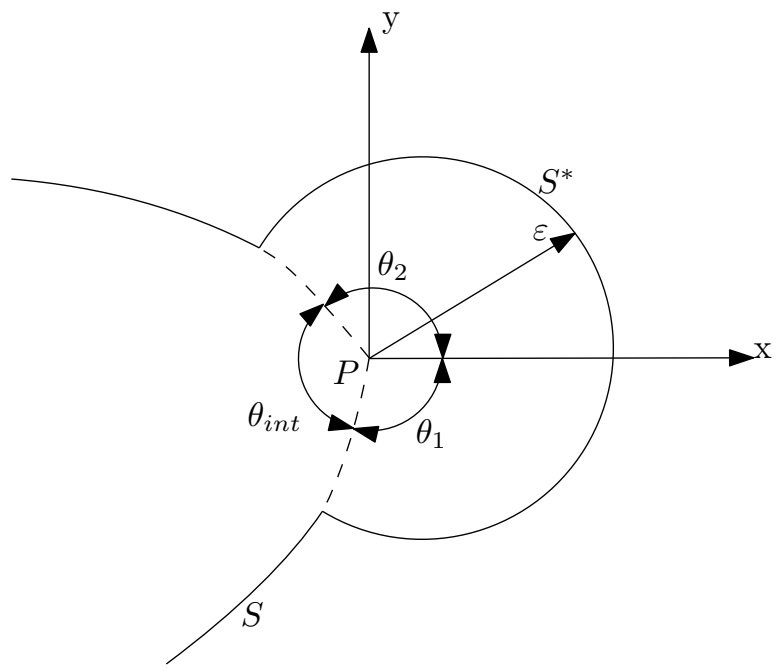

Figura 2.2: Contorno modificado.

zero, satisfaz essa exigência. Contudo a escolha que se mostra mais adequada é a função cujo laplaciano é o delta de Dirac (BREBBIA, 1982):

$$
\nabla^{2} \omega=\Delta(P, Q)
$$

o que implica na utilização da solução fundamental desse problema como função peso, ou seja, $\omega=u^{*}$. Então tem-se:

$$
\int_{s} \frac{\partial u}{\partial n} u^{*} d s-\int_{s} u \frac{\partial u^{*}}{\partial n} d s+\int_{A} u \Delta(P, Q) d A
$$

Tomando o ponto fonte no interior do domínio $A$, pela propriedade do delta de Dirac, tem-se:

$$
\begin{gathered}
\int_{s} \frac{\partial u}{\partial n} u^{*} d s-\int_{s} u \frac{\partial u^{*}}{\partial n} d s+u(P)=0 . \\
u(P)=\int_{s} u \frac{\partial u^{*}}{\partial n} d s-\int_{s} \frac{\partial u}{\partial n} u^{*} d s .
\end{gathered}
$$

A Eq.2.34 é a equação integral de contorno quando o ponto fonte encontra-se no interior do domínio.

A fim de considerar o ponto $P$ no contorno, faz-se uma pequena modificação no mesmo, conforme a figura 2.2 .

Assim, tem-se:

$$
u(P)=\int_{s-s^{*}} u \frac{\partial u^{*}}{\partial n} d s-\int_{s-s^{*}} u^{*} \frac{\partial u}{\partial n} d s+\int_{s^{*}} u \frac{\partial u^{*}}{\partial n} d s-\int_{s^{*}} u^{*} \frac{\partial u}{\partial n} d s .
$$

Considerando que que $s^{*}$ é um arco de circunferência com centro em $P$, tem-se que 
$\frac{\partial u^{*}}{\partial n}=-\frac{\partial u^{*}}{\partial r}$ no contorno modificado bidimensional, portanto:

$$
\int_{s^{*}} u \frac{\partial u^{*}}{\partial n} d s=-\int_{-\theta_{1}}^{\theta_{2}} u \frac{\partial u^{*}}{\partial r} \varepsilon d \theta=-\int_{-\theta_{1}}^{\theta_{2}} u \frac{1}{2 \pi r} \varepsilon d \theta
$$

Observando que $r=\varepsilon$ para qualquer $\theta$, e fazendo $\varepsilon$ tender a $0, u$ assume o valor de $u(P)$. Por fim, tem-se:

$$
\int_{s} u \frac{\partial u^{*}}{\partial n} d s=-\frac{u(P)\left(\theta_{2}+\theta_{1}\right)}{2 \pi}
$$

A mesma análise deve ser feita para:

$$
\int_{s^{*}} u^{*} \frac{\partial u}{\partial n} d s=\int_{-\theta_{1}}^{\theta_{2}} \frac{-1}{2 \pi k} \log r \frac{\partial u}{\partial n} r d \theta .
$$

Como $r=\varepsilon=$ constante, tem-se:

$$
\int_{s^{*}} u^{*} \frac{\partial u}{\partial n} d s=\frac{-1}{2 \pi k} \varepsilon \log \varepsilon \int_{-\theta_{1}}^{\theta_{2}} \frac{\partial u}{\partial n} d \theta
$$

Fazendo $\varepsilon$ tender a zero, tem-se:

$$
\begin{gathered}
\int_{s^{*}} u^{*} \frac{\partial u}{\partial n} d s=\frac{-1}{2 \pi k} \lim _{\varepsilon \rightarrow 0} \varepsilon \log \varepsilon\left(\theta_{2}+\theta_{1}\right), \\
\int_{s^{*}} u^{*} \frac{\partial u}{\partial n} d s=0 .
\end{gathered}
$$

Voltando a equação original, segue:

$$
\begin{gathered}
u(P)=\int_{s} \frac{\partial u}{\partial n} u^{*} d s-\int_{s} u \frac{\partial u^{*}}{\partial n} d s+\frac{u(P)\left(\theta_{2}+\theta_{1}\right)}{2 \pi}-0, \\
u(P)\left(\frac{2 \pi-\left(\theta_{2}+\theta_{1}\right)}{2 \pi}\right)=\int_{s} \frac{\partial u}{\partial n} u^{*} d s-\int_{s} u \frac{\partial u^{*}}{\partial n} d s .
\end{gathered}
$$

Conforme a Fig.(2.2), $\theta_{\text {int }}$ é o ângulo interno do contorno dado por $\theta_{\text {int }}=2 \pi-\left(\theta_{1}+\right.$ $\left.\theta_{2}\right)$.

Assim:

$$
\frac{\theta_{\text {int }}}{2 \pi} u(P)=\int_{s} \frac{\partial u}{\partial n} u^{*} d s-\int_{s} u \frac{\partial u^{*}}{\partial n} d s
$$

que é a integral de contorno quando o ponto fonte pertence ao contorno.

Quando o ponto fonte não pertence ao contorno nem ao domínio, devido à propriedade do delta de Dirac, tem-se:

$$
\int_{s} \frac{\partial u}{\partial n} u^{*} d s-\int_{s} u \frac{\partial u^{*}}{\partial n} d s=0 .
$$


Generalizando, a equação integral de contorno pode ser escrita como

$$
c u(P)=\int_{s} \frac{\partial u}{\partial n} u^{*} d s-\int_{s} u \frac{\partial u^{*}}{\partial n} d s
$$

onde

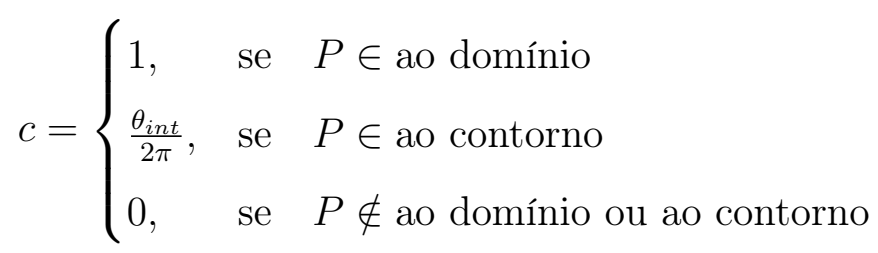

Quando o ponto fonte encontra-se em ponto suave do contorno, isto é, não é um canto, tem-se:

$$
c=\frac{\theta_{\text {int }}}{2 \pi}=\frac{\pi}{2 \pi}=\frac{1}{2} .
$$

Fazendo os mesmos passos para um problema tridimensional, obtém-se

$$
\int_{s^{*}} u \frac{\partial u^{*}}{\partial n} d s=-\int_{s^{*}} u \frac{\partial u^{*}}{\partial r} \varepsilon^{2} \sin \theta d \theta d \varphi=\int_{s^{*}} u \frac{1}{4 \pi r^{2}} \varepsilon^{2} \sin \theta d \theta d \varphi=\frac{u(P) \theta_{\text {sol }}}{4 \pi}
$$

$\mathrm{e}$

$$
\int_{s^{*}} u^{*} \frac{\partial u}{\partial n} d s=-\int_{s^{*}} u^{*} \frac{\partial u}{\partial r} \varepsilon^{2} \sin \theta d \theta d \varphi=-\int_{s^{*}} u^{*} \frac{1}{4 \pi r} \varepsilon^{2} \sin \theta d \theta d \varphi=0 .
$$

onde $\theta_{\text {sol }}$ é o angulo sólido da esfera com origem no ponto fonte.

A equação geral de contorno pode então ser escrita da mesma maneira do caso bidimensional, equação (2.37), alterando apenas os valores de $c$ :

$$
c= \begin{cases}1, & \text { se } \quad P \in \text { ao domínio } \\ \frac{4 \pi-\theta_{\text {sol }},}{4 \pi}, & \text { se } \quad P \in \text { ao contorno } \\ 0, & \text { se } \quad P \notin \text { ao domínio ou ao contorno }\end{cases}
$$

\subsubsection{Derivada da solução fundamental}

A equação 2.37 depende da derivada da solução fundamental $\frac{\partial u^{*}}{\partial n}$. Uma maneira conveniente de escrever essa função, em duas dimensões, é:

$$
\frac{\partial u^{*}}{\partial n}=\frac{\partial u^{*}}{\partial r} \frac{\partial r}{\partial n}=\frac{-1}{2 \pi r} \frac{\partial r}{\partial n}
$$

e em três dimensões:

$$
\frac{\partial u^{*}}{\partial n}=\frac{\partial u^{*}}{\partial r} \frac{\partial r}{\partial n}=\frac{-1}{4 \pi r^{2}} \frac{\partial r}{\partial n}
$$




\subsection{Problema elástico}

O problema geral de elasticidade pode ser representado conforme a figura 2.1, onde $\Gamma_{1}$ corresponde às condições com os deslocamentos conhecidos e $\Gamma_{2}$ às condições com as forças de superfícies conhecidas. Usa-se a notação indicial para representar as equações de maneira compacta. No caso bidimensional os índices subscritos variam de 1 a 2 e enquanto no caso tridimensional eles variam de 1 a 3 . A derivada parcial em relação a uma coordenada $x_{i}$ é dada por uma vírgula subscrita como $a_{, i}=\frac{\partial a}{\partial x_{i}}$ e $a_{n}$ é a derivada em relação à normal da superfície. Índices repetidos indicam soma ao menos que o inverso seja afirmado. Para o caso estático, o somatório das forças aplicadas ao corpo tem de ser zero. O que pode ser escrito pela seguinte equação integral:

$$
\int_{\Gamma} t_{i} d \Gamma+\int_{\Omega} b_{i} d \Omega=0
$$

onde $t_{i}$ são as forças de superfície e $b_{i}$ são as forças de corpo. A equação 2.41 contém tanto em integrais de superfície quanto de volume.

O teorema de Green relaciona uma integral de volume a uma de superfície:

$$
\int_{\Omega} g_{i, i} d \Omega=\int_{\Gamma} g_{i} n_{i} d \Gamma
$$

onde $g_{i}$ é uma função arbitrária com derivada primeira contínua. Considerando também a transformação de tensão de Cauchy:

$$
t_{i}=\sigma_{i j} n_{j}
$$

onde $\sigma_{i j}$ são as componentes do tensor de tensões, pode-se rescrever a equação de equilíbrio estático de maneira conveniente, onde todas as integrais são de domínio:

$$
\int_{\Omega} \sigma_{i j, j} d \Omega+\int_{\Omega} b_{i} d \Omega=0
$$

\subsubsection{Teorema de Betti}

O teorema de Betti é baseado no princípio do trabalho virtual. Dado um corpo com dois estados distintos de tensão-deformação $\sigma_{i j}^{(1)}, \epsilon_{i j}^{(1)}$ e $\sigma_{i j}^{(2)}, \epsilon_{i j}^{(2)}$, o teorema diz que o trabalho realizado pelas tensões do sistema (1) nos deslocamentos do sistema (2) tem de ser equivalente ao trabalho realizado pelas tensões do sistema (2) nos deslocamentos do sistema (1). Isso pode ser escrito matematicamente da seguinte forma:

$$
\int_{\Omega} \sigma_{i j}^{(1)} \epsilon_{i j}^{(2)} d \Omega=\int_{\Omega} \sigma_{i j}^{(2)} \epsilon_{i j}^{(1)} d \Omega
$$


que pode ser reescrita, por meio da relação deformação-deslocamento:

$$
\epsilon_{i j}=\frac{1}{2}\left(u_{i, j}+u_{j, i}\right)
$$

como:

$$
\frac{1}{2} \int_{\Omega} \sigma_{i j}^{(1)}\left(u_{i, j}+u_{j, i}\right)^{(2)} d \Omega=\frac{1}{2} \int_{\Omega} \sigma_{i j}^{(2)}\left(u_{i, j}+u_{j, i}\right)^{(1)} d \Omega .
$$

Devido a simetria do tensor de tensões e ao somatório relativo aos índices repetidos, a equação pode ser reescrita da seguinte forma:

$$
\int_{\Omega} \sigma_{i j}^{(1)}\left(u_{i, j}\right)^{(2)} d \Omega=\int_{\Omega} \sigma_{i j}^{(2)}\left(u_{i, j}\right)^{(1)} d \Omega
$$

Usando a regra do produto

$$
\begin{gathered}
\left(\sigma_{i j} u_{i}\right)_{, j}=\left(\sigma_{i j}\right)_{, j} u_{i}+\sigma_{i j}\left(u_{i}\right)_{, j} \\
\sigma_{i j}\left(u_{i}\right)_{, j}=\left(\sigma_{i j} u_{i}\right)_{, j}-\left(\sigma_{i j}\right)_{, j} u_{i}
\end{gathered}
$$

pode-se reescrever o lado esquerdo da equação (2.48) como:

$$
\int_{\Omega} \sigma_{i j}^{(1)}\left(u_{i, j}\right)^{(2)} d \Omega=\int_{\Omega}\left[\left(\sigma_{i j}^{(1)}\left(u_{i}\right)^{(2)}\right)_{, j}-\sigma_{i j, j}^{(1)}\left(u_{i}\right)^{(2)}\right] d \Omega
$$

O segundo termo do lado direito da equação pode ser escrito como as forças de corpo, conforme a equação 2.44:

$$
\int_{\Omega} \sigma_{i j}^{(1)}\left(u_{i, j}\right)^{(2)} d \Omega=\int_{\Omega}\left(\sigma_{i j}^{(1)}\left(u_{i}\right)^{(2)}\right)_{, j} d \Omega+\int_{\Omega} b_{i}^{(1)}\left(u_{i}\right)^{(2)} d \Omega .
$$

Aplicando o teorema da divergência a primeira integral do lado direito, obtém-se:

$$
\int_{\Omega} \sigma_{i j}^{(1)}\left(u_{i, j}\right)^{(2)} d \Omega=\int_{\Gamma}\left(\sigma_{i j}^{(1)}\left(u_{i}\right)^{(2)}\right) n_{j} d \Gamma+\int_{\Omega} b_{i}^{(1)}\left(u_{i}\right)^{(2)} d \Omega
$$

A forma final do teorema de Betti é obtida ao realizar esse procedimento nos dois lados da equação:

$$
\int_{\Gamma}\left(\sigma_{i j}^{(1)}\left(u_{i}\right)^{(2)}\right) n_{j} d \Gamma+\int_{\Omega} b_{i}^{(1)}\left(u_{i}\right)^{(2)} d \Omega=\int_{\Gamma}\left(\sigma_{i j}^{(2)}\left(u_{i}\right)^{(1)}\right) n_{j} d \Gamma+\int_{\Omega} b_{i}^{(2)}\left(u_{i}\right)^{(1)} d \Omega
$$


e aplicar a transformação de Cauchy, dada pela equação (2.43):

$$
\int_{\Gamma} t_{i}^{(1)} u_{i}^{(2)} d \Gamma+\int_{\Omega} b_{i}^{(1)} u_{i}^{(2)} d \Omega=\int_{\Gamma} t_{i}^{(2)} u_{i}^{(1)} d \Gamma+\int_{\Omega} b_{i}^{(2)} u_{i}^{(1)} d \Omega
$$

\subsubsection{Equação integral de contorno}

A equação 2.55 contém tanto integrais de superfícies quanto de domínio. Essa equação, sob hipóteses específicas, pode ser transformada numa equação integral de contorno. Considere, sem perda de generalidade, o sistema (1) como o problema a ser resolvido e o sistema (2) como um estado de tensão arbitrário usado para facilitar a solução do problema. O sistema (2) corresponde a um meio infinito, homogêneo, isotrópico e elástico sob um carregamento pontual. Esse carregamento pontual é representado pelo delta de Dirac que considera uma força de corpo $b_{i}$. Aplicando as propriedades do delta de Dirac ao último termo da forma final do teorema de Betti 2.55, obtém-se:

$$
\int_{\Omega} b_{i}^{(2)} u_{i}^{(1)} \Omega=\int_{\Omega} \Delta(p, x) e_{i} u_{i}^{(1)} \Omega=u_{i}^{(1)}(p) e_{i} .
$$

O sistema (1) contém justamente as variáveis desconhecidas. Portanto, considerando as forças de campo nulas, pode-se dizer que:

$$
u_{i}^{(1)}=u_{j}(Q), t_{i}^{(1)}=t_{j}(Q), b_{i}^{(1)}=0 .
$$

Enquanto as variáveis do sistema (2) são as respostas relativas ao carregamento pontual, podendo ser escritas da seguinte forma:

$$
u_{i}^{(2)}=U_{i j}(p, Q) e_{j}, t_{i}^{(2)}=T_{i j}(p, Q) e_{j}
$$

onde $Q$ é um ponto no contorno, $p$ um ponto no domínio e $U_{i j}$ e $T_{i j}$ são as soluções fundamentais. Ao substituir esses termos na equação (2.55) obtém-se a identidade de Somigliana para deslocamentos:

$$
u_{i}(p)+\int_{\Gamma} T_{i j}(p, Q) u_{j}(Q) d \Gamma=\int_{\Gamma} U_{i j}(p, Q) t_{j}(Q) d \Gamma
$$

Essa equação relaciona os deslocamentos de um ponto interno $p$ com os valores de deslocamento e forças de superfície no contorno. Ainda é necessário encontrar as soluções fundamentais desse problema, que é explorado a seguir. 


\subsubsection{Soluções fundamentais}

O primeiro passo na obtenção da solução fundamental é encontrar a equação de Navier para a elasticidade. Partindo da relação tensão-deformação:

$$
\sigma_{i j}=\frac{2 \mu \nu}{1-2 \nu} \delta_{i j} \varepsilon_{m m}+2 \mu \varepsilon_{i j}
$$

onde $\varepsilon_{i j}$ é o tensor de deformações, $\mu$ é o módulo de cisalhamento e $\nu$ o coeficiente de Poisson, e usando a relação deformação-deslocamento (2.46), obtém-se:

$$
\sigma_{i j}=\frac{2 \mu \nu}{1-2 \nu} \delta_{i j} u_{m, n}+\mu\left(u_{i, j}+u_{j, i}\right)
$$

onde $\delta$ é o delta de Kronecker. Pode-se substituir essa relação na equação de equilíbrio:

$$
\sigma_{i j, j}+b_{i}=0
$$

e obter a equação de Navier para deslocamentos:

$$
-\frac{b_{i}}{\mu}=\frac{1}{1-2 \nu} u_{i, i j}+u_{i, j j}
$$

Essa relação, apesar de ter exatamente três incógnitas e três equações diferencias parciais, os três deslocamentos, que são justamente as incógnitas, estão acoplados, o que torna difícil encontrar uma solução analítica. A estratégia empregada consiste em expressar o vetor de deslocamentos em termo de outro vetor $G_{i}$ que satisfaz a equação de Navier.

$$
u_{i}=G_{i, j j}-\frac{1}{2(1-\nu)} G_{j, i j}
$$

onde $G_{i}$ é o vetor de Galerkin. Substituindo na equação 2.63), obtém-se:

$$
G_{i, j j k k}=\frac{-b_{i}}{\mu}
$$

A nova equação, depois de ter os componentes de deslocamentos expressados em termo do vetor de Galerkin, é desassociada. Isso é evidente pela presença de somente um termo livre $i$. O operador que o vetor de Galerkin está submetido é um operador bi-harmônico que é amplamente estudado em análise (ZWILLINGER, 1998).

Considerando o carregamento como uma força pontual, visto que se procura a solução fundamental, obtém-se:

$$
G_{i, j j k k} \mu+\Delta(p, Q) e_{i}=0
$$


A solução desse equação é conhecida como solução de Kelvin e é dada, em três dimensões, por:

$$
G_{i}=\frac{1}{8 \pi(1-\nu)} r(p, Q) e_{i}
$$

onde $r(p, Q)$ é a distância entre o ponto fonte $p$ e o ponto fonte $Q$. Substituindo a equação 2.67) na equação (2.64), encontra-se a solução fundamental de deslocamentos:

$$
U_{i j}(p, Q)=\frac{1}{16 \mu \pi(1-\nu)} \frac{1}{r(p, Q)}\left[(3-4 \nu) \delta_{i j}+r_{, i}(p, Q) r_{, j}(p, Q)\right],
$$

onde $U_{i j}(p, Q)$ representa o deslocamento num ponto $Q$ na direção $j$ devido a um carregamento pontual na direção $i$ num ponto $p$.

A equação 2.61 relaciona a tensão com o deslocamento e com ela pode-se transformar a solução fundamental de deslocamento 2.68 na solução fundamental de forças de superfície:

$$
\begin{aligned}
T_{i j}(p, Q)=\frac{-1}{8 \pi(1-\nu) r^{2}(p, Q)} r_{, n}[(1-2 \nu) & \left.\delta_{i j}+3 r_{, i}(p, Q) r_{, j}(p, Q)\right] \\
& -(1-2 \nu)\left[r_{, j}(p, Q) n_{i}-r_{, i}(p, Q) n_{j}\right]
\end{aligned}
$$

onde o termo $n_{j}$ denota as componentes da normal no ponto campo $Q$ e $T_{i j}(p, Q)$ representa a força de superfície num ponto $Q$ na direção $j$ devido a um carregamento pontual na direção $i$ num ponto $p$.

As soluções fundamentais bidimensionais são obtidas de maneira análoga. O vetor de Galerkin para duas dimensões é

$$
G_{i}=\frac{1}{8 \pi(1-\nu)} r^{2} \log (1 / r) e_{i}
$$

e soluções fundamentais de deslocamento e forças de superfície, para estado plano de deformação, são dadas por:

$$
\begin{gathered}
U_{i j}(p, Q)=\frac{1}{8 \mu \pi(1-\nu)}\left[(3-4 \nu) \log (1 / r) \delta_{i j}+r_{, i} r_{, j}\right] \\
T_{i j}(p, Q)=\frac{-1}{4 \pi(1-\nu) r} r_{, n}\left[(1-2 \nu) \delta_{i j}+2 r_{, i} r_{, j}\right]+\frac{(1-2 \nu)}{4 \pi(1-\nu) r}\left[r_{, j} n_{i}-r_{, i} n_{j}\right] .
\end{gathered}
$$

As soluções fundamentais dependem somente de propriedades materiais e da distância entre pontos fonte e campo e, portanto, podem ser facilmente calculadas. A equação 2.59), reescrita abaixo:

$$
u_{i}(p)+\int_{\Gamma} T_{i j}(p, Q) u_{j}(Q) d \Gamma=\int_{\Gamma} U_{i j}(p, Q) t_{j}(Q) d \Gamma
$$




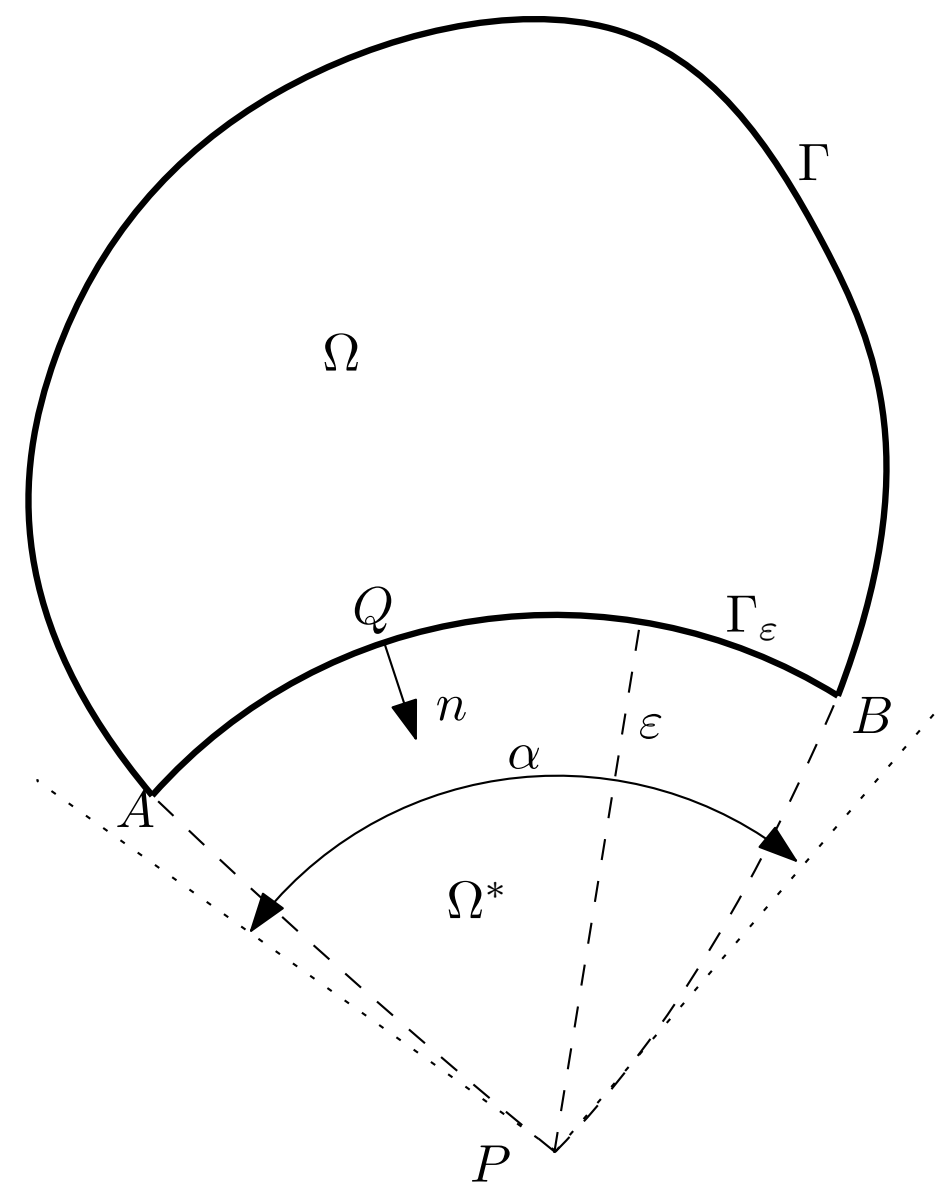

Figura 2.3: Alteração da geometria para consideração da singularidade no contorno

contém apenas termos presentes no contorno com exceção do primeiro termo que consiste num ponto fonte dentro do domínio. Para que todas as grandezas estejam no contorno, o ponto fonte $p$ também tem de estar no contorno e será chamado agora de $P$. Devido à presença de funções singulares nas soluções fundamentais, as integrais precisam de um tratamento especial, tanto em duas quanto em três dimensões. Esse tratamento consiste em dividir o domínio em duas partes: uma contendo a parte singular e outra não. A superfície alterada tem forma semicircular ou hemisférica centrada no ponto singular $P$, conforme pode ser observado, no caso bidimensional, na figura 2.3 .

No novo domínio em análise, o ponto fonte não está dentro do domínio o que faz com que o primeiro termo da equação 2.59 desapareça. Essa pode ser rescrita, levando em conta as novas superfícies, como:

$$
\begin{aligned}
\int_{\Gamma-\Gamma_{\varepsilon}} T_{i j}(p, Q) u_{j}(Q) d \Gamma+\int_{\Gamma_{\varepsilon}} T_{i j}(p, Q) u_{j}(Q) d \Gamma= \\
\\
\int_{\Gamma-\Gamma_{\varepsilon}} U_{i j}(p, Q) t_{j}(Q) d \Gamma+\int_{\Gamma_{\varepsilon}} U_{i j}(p, Q) t_{j}(Q) d \Gamma .
\end{aligned}
$$


O próximo passo é tomar o limite dessas integrais para $\varepsilon \rightarrow 0$. As integrais de superfície em $\Gamma-\Gamma_{\varepsilon}$ se torna integrais em $\Gamma$, restando examinar as integrais em $\Gamma_{\varepsilon}$ :

$$
\lim _{\varepsilon \rightarrow 0} \int_{\Gamma_{\varepsilon}} T_{i j}(p, Q) u_{j}(Q) d \Gamma
$$

$\mathrm{e}$

$$
\lim _{\varepsilon \rightarrow 0} \int_{\Gamma_{\varepsilon}} U_{i j}(p, Q) t_{j}(Q) d \Gamma .
$$

A solução fundamental $U_{i j}(p, Q)$ pode ser separada em uma parte singular de ordem $\log (r)$ e em uma parte não singular que tem limite igual a 0 . Analisando primeiramente somente a parte singular, obtém-se:

$$
\lim _{\varepsilon \rightarrow 0} \int_{\Gamma_{\varepsilon}} \log (r) d \Gamma=\lim _{\varepsilon \rightarrow 0} \int_{0}^{\alpha} \log (\varepsilon) \varepsilon d \alpha=\alpha \lim _{\varepsilon \rightarrow 0}(\varepsilon \log \varepsilon)=0
$$

Levando esse limite em consideração e que o ponto $Q$ tende ao ponto $P$ a medida que $\varepsilon$ tende a zero, pode-se dizer que:

$$
\lim _{\varepsilon \rightarrow 0} \int_{\Gamma_{\varepsilon}} U_{i j}(p, Q) t_{j}(Q) d \Gamma=C \lim _{\varepsilon \rightarrow 0} t_{i}(P) \int_{\Gamma_{\varepsilon}} \log (r) d \Gamma=0
$$

Analisando agora o núcleo singular $1 / r$ da solução fundamental $T_{i j}(p, Q)$ :

$$
\lim _{\varepsilon \rightarrow 0} \int_{\Gamma_{\varepsilon}} \frac{1}{r} d \Gamma=\lim _{\varepsilon \rightarrow 0} \int_{0}^{\alpha} \frac{1}{\varepsilon} \varepsilon d \alpha=\alpha
$$

É interessante notar que, mesmo com o limite do comprimento indo para zero, essa integral de linha tem seu limite diferente de zero. Isso ocorre somente porque a integral é singular e é calculada no sentido do valor principal de Cauchy. Na solução fundamental, o núcleo singular está multiplicado por diferentes derivadas de $r$ que alteram o valor da integral, mas continuam sendo não-nulas.

Considerando esse limite não-nulo e o fato que o ponto $Q$ tende ao ponto $P$ a medida que $\varepsilon$ tende a zero, pode-se dizer que:

$$
\lim _{\varepsilon \rightarrow 0} \int_{\Gamma_{\varepsilon}} T_{i j}(p, Q) u_{j}(Q) d \Gamma=C \lim _{\varepsilon \rightarrow 0} u_{i}(P) \int_{\Gamma_{\varepsilon}} \frac{1}{r} d \Gamma=C_{i j} U_{j}(P) .
$$

onde o termo livre $C_{i j}$ depende da geometria do problema e para pontos suaves é igual a $\frac{1}{2} \delta_{i j}$, como foi mostrado detalhadamente para o caso potencial na seção anterior. A identidade de Somigliana pode então ser reescrita com grandezas somente no contorno 
da seguinte maneira:

$$
C_{i j} u_{j}(P)+\int_{\Gamma} T_{i j}(P, Q) u_{j}(Q) d \Gamma=\int_{\Gamma} U_{i j}(P, Q) t_{j}(Q) d \Gamma
$$

Essa é a equação normalmente usada no MEC mesmo com suas integrais sendo singulares. Uma alternativa é usar a hipótese de deslocamento de corpo rígido. Essa hipótese assume que o corpo está livre de forças de superfície se todos seus pontos tiverem os mesmos deslocamentos. Nessa situação a equação 2.80 se reduz a:

$$
C_{i j} u_{j}(P)=-\int_{\Gamma} T_{i j}(P, Q) u_{i}(P) d \Gamma
$$

Substituindo esse resultado na equação anterior, obtém-se:

$$
\int_{\Gamma} T_{i j}(P, Q)\left(u_{j}(Q)-u_{i}(P)\right) d \Gamma=\int_{\Gamma} U_{i j}(P, Q) t_{j}(Q) d \Gamma
$$

Essa equação é conhecida como uma equação auto-regularizada RICHARDSON; CRUSE, 1999). Nesse tipo de equação, somente integrais fracamente singulares tem de ser calculadas, evitando a avaliação das integrais no valor principal de Cauchy ou na parte finita de Hadamard. Esse processo de regularização não modifica o significado da equação integral de contorno original, uma vez que trata-se apenas de uma manipulação algébrica da mesma (CRUSE; RICHARDSON, 1996).

\subsection{Discretização}

O objeto de análise desse trabalho é a formulação isogeométrica. No entanto, também é importante mostrar o funcionamento do método convencional pois o mesmo será usado em comparações com os resultados produzidos pelas formulações apresentadas neste trabalho. O contorno $\Gamma$, que delimita o domínio $\Omega$ do problema apresentado na figura 2.1, é dividido em um número finito $M$ de segmentos, denominados elementos de contorno. O contorno real é portanto substituído por um contorno aproximado, quando trata-se do método convencional, baseado em esquemas de interpolação previamente definidos. Da mesma forma, as grandezas do contorno (deslocamentos e forças de superfície) são aproximadas por funções de interpolação definidas em termos de seus valores nodais no contorno.

Os elementos de contorno podem ser contínuos ou descontínuos e ter diferentes esquemas de interpolação como, por exemplo, constantes, lineares, quadráticos, cúbicos e quárticos. Para o caso bidimensional a interpolação das variáveis desconhecida se dá ao longo de uma curva. Assim, as funções de interpolação, ou funções de forma, são 
polinômios de um parâmetro apenas. No caso tridimensional, as funções de forma irão depender de dois parâmetros distintos por se tratar de uma superfície. Os deslocamentos, forças de superfície e coordenadas do contorno, em duas dimensões, são descritos portanto pelas seguintes relações:

$$
\begin{array}{r}
u_{k}(Q)=u_{k}(\xi)=\sum_{i=1}^{m} N_{i} u_{k}^{i} \\
t_{k}(Q)=t_{k}(\xi)=\sum_{i=1}^{m} N_{i} t_{k}^{i} \\
x_{k}(Q)=x_{k}(\xi)=\sum_{i=1}^{m} N_{i} x_{k}^{i}
\end{array}
$$

onde $u_{k}^{i}, t_{k}^{i}, x_{k}^{i}$ são, respectivamente, os valores do deslocamento, força de superfície e coordenadas dos pontos nodais do contorno. O parâmetro $m$ presente no somatório corresponde ao número de pontos nodais do elemento assumindo os valores 2, 3, 4 e 5 para elementos lineares, quadráticos, cúbicos e quárticos, respectivamente. Na equação (2.83, $N_{i}$ representa as funções de interpolação em termos da coordenada intrínseca $\xi$ no caso $2 \mathrm{D}$ e das coordenadas $\xi$ e $\eta$ no caso $3 \mathrm{D}$.

Com intuito de possibilitar a avaliação numérica das integrais presentes nas equações integrais de contorno, faz-se necessária a transformação das coordenadas cartesianas globais dos elementos em coordenadas adimensionais locais. As coordenadas cartesianas dos nós dos elementos são parametrizadas em coordenadas adimensionais que assumem valores entre -1 e 1, o que possibilita a utilização imediata da quadratura de Gauss padrão. O jacobiano da transformação de coordenadas adimensionais intrínsecas $\xi$ para coordenadas globais $x_{i}$ é dado, em duas dimensões, por:

$$
J(\xi)=\sqrt{\left(\frac{\partial x_{2}}{\partial \xi}\right)^{2}+\left(\frac{\partial x_{1}}{\partial \xi}\right)^{2}}
$$

e em três dimensões por:

$$
J(\xi, \eta)=\left\|x_{i, \xi} \times x_{j, \eta}\right\|
$$

Uma atenção especial deve ser dada às junções entre elementos quando da discretização do contorno do problema. As componentes do deslocamento tem valores únicos nas interseções entre elementos. Entretanto, o mesmo não pode ser assegurado para as componentes da força de superfície. Podem existir descontinuidades na força de superfície em pontos onde a normal ao contorno não seja única (figura 2.4. Mesmo em partes suaves do contorno, a força de superfície pode apresentar descontinuidades (figura 2.5), o que não é raro ocorrer em casos práticos. O algoritmo do MEC implementado permite a existência de descontinuidades da normal ou das forças de superfície 


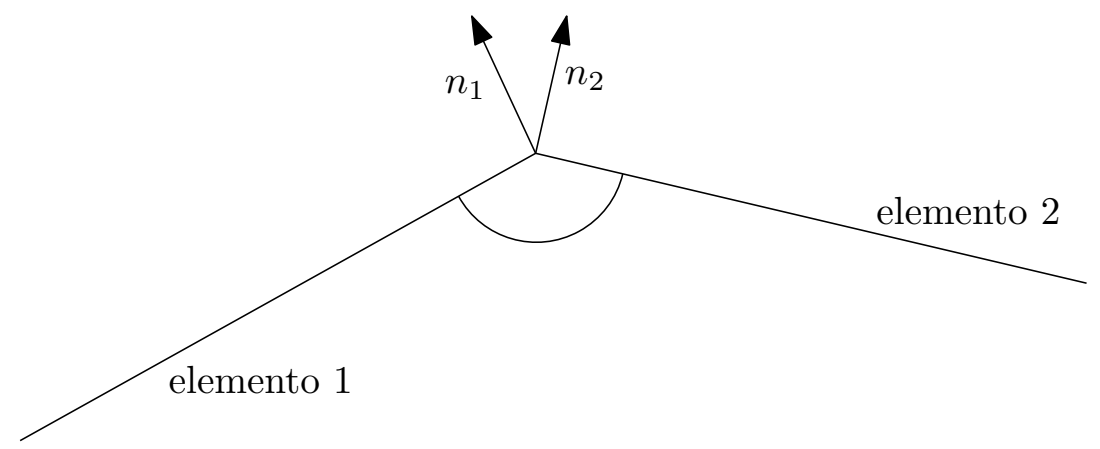

Figura 2.4: Descontinuidade da normal no contorno

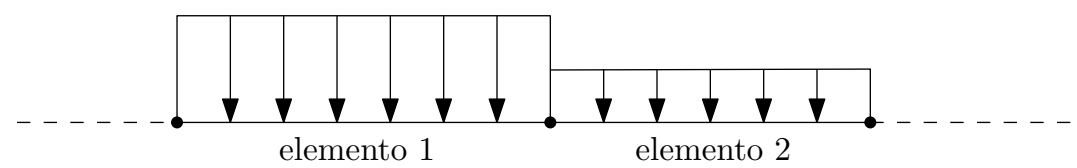

Figura 2.5: Descontinuidade da força de superfície

utilizando um único nó nas junções entre elementos. É possível prescrever diferentes valores de força de superfície nos elementos anterior e posterior ao nó compartilhado. Pode-se ainda prescrever a força de superfície na extremidade de um elemento e o deslocamento na extremidade do outro elemento. Deve-se apenas manter a relação de uma incógnita por nó.

A forma discretizada em termos de deslocamentos é obtida com base na equação 2.80 e é dada por:

$$
C_{i j} u_{j}(P)+\sum_{k=1}^{M} \int_{\Gamma_{k}} T_{i j}(P, Q) u_{i}^{k}(Q) d \Gamma=\sum_{k=1}^{M} \int_{\Gamma_{k}} U_{i j}(P, Q) t_{i}^{k}(Q) d \Gamma .
$$

sendo $M$ o número de elementos, $\Gamma_{i}$ o contorno do elemento $i$.

Quando o ponto de integração está sobre o elemento a ser integrado, a primeira integral da equação 2.86 se torna fortemente singular e a segunda integral fracamente singular. Devido à presença do núcleo fortemente singular, a primeira integral na equação 2.86 deverá ser interpretada no sentido do valor principal de Cauchy. A segunda integral por sua vez não necessita de interpretação especial. Entretanto, devese tomar cuidado no sentido da correta avaliação desta integral, tendo em vista a natureza logarítmica da solução fundamental.

A avaliação numérica da primeira integral da equação 2.86 pode ser feita através de esquema de quadratura de Gauss padrão, exceto para o elemento que contém o ponto fonte, que deverá ser avaliada de forma diferenciada. Essa integral pode ser calculada por meio de uma expansão assintótica (GUIGGIANI et al., 1992), considerações de deslocamento de corpo rígido (BREBBIA, 1982) ou uma integração numérica especial (KUTT, 1975). Enquanto a integral singular do lado direito pode ser calculada através 
de integração logarítmica ou através da transformação proposta por (TELLES, 1987). Uma alternativa para se evitar a presença de integrais singulares na formulação padrão é a utilização de pontos de colocação fora do domínio.

A equação 2.86 pode ser aproximada usando a interpolação desejada e escrita em termo das valores nodais e das funções de interpolação $N_{i}$ :

$$
\begin{aligned}
& \int_{\Gamma_{i}} T_{i j}(P, Q) u_{j}(Q) d \Gamma=\int_{-1}^{1} T_{i j}(P, Q)\left(\sum_{k=1}^{m} N_{k} u_{i}^{k}\right) J(\xi) d \xi \\
& \int_{\Gamma_{l}} U_{i j}(P, Q) t_{j}(Q) d \Gamma=\int_{-1}^{1} U_{i j}(P, Q)\left(\sum_{k=1}^{m} N_{k} t_{i}^{k}\right) J(\xi) d \xi
\end{aligned}
$$

resultando em:

$$
\begin{aligned}
\sum_{k=1}^{m} C_{i j} u_{j}^{k}(P)+\sum_{k=1}^{M} \sum_{k=1}^{m}\left(u_{i}^{k} \int_{-1}^{1} T_{i j}(P, Q) N_{k} J(\xi) d \xi\right)= \\
\sum_{k=1}^{M} \sum_{k=1}^{m}\left(t_{i}^{k} \int_{-1}^{1} U_{i j}(P, Q) N_{k} J(\xi) d \xi\right)
\end{aligned}
$$

A equação pode ser escrita de forma compacta, da seguinte forma:

$$
\sum_{k=1}^{m} C_{i j} u_{j}^{k}(P)+\sum_{k=1}^{M} \sum_{k=1}^{m} u_{j}^{k} H_{i j}^{k}=\sum_{k=1}^{M} \sum_{k=1}^{m} t_{j}^{k} G_{i j}^{k},
$$

onde $H_{i j}^{\prime}$ e $G_{i j}$ são as integrais da equação 2.89 . Fazendo

$$
H_{i j}=H_{i j}^{\prime}+C_{i j}
$$

nas entradas relativas ao ponto fonte, torna-se possível realizar a colocação em todos os nós do contorno e obter um sistema com $N$ equações, onde $N$ é o número de graus de liberdade, que pode ser escrito de forma matricial por:

$$
\mathbf{H u}=\mathbf{G t}
$$

onde $\mathbf{H}$ e $\mathbf{G}$ são matrizes quadradas de tamanho $N$ e $\mathbf{u}$ e $\mathbf{t}$ são vetores com os valores nodais de deslocamentos e forças de superfície.

Substituindo as condições de contorno na equação matricial 2.92), obtém-se um sistema linear que pode ser escrito como:

$$
\mathrm{Ax}=\mathrm{b}
$$


onde $\mathbf{x}$ é o vetor que contém os deslocamentos e forças de superfície desconhecidos, A é uma matriz de coeficientes que é cheia e não simétrica e b é obtido por meio da multiplicação dos valores conhecidos pelas colunas correspondentes de $\mathbf{H}$ e $\mathbf{G}$.

O sistema pode ser enfim resolvido usando um processo de solução direto ou um iterativo. No entanto, a solução e o armazenamento da matriz A ficam caros para problemas maiores. Na tentativa de superar esse problema, esse trabalho propõe o uso do ACA, que será detalhado no próximo capítulo. 


\section{MATRIZES HIERÁRQUICAS E APROXIMAÇÃO CRUZADA ADAPTATIVA}

\subsection{Introdução}

O método dos elementos de contorno gera matrizes cheias, o que torna problemas de larga escala custosos tanto em custo de armazenamento quanto em tempo gasto nas suas operações. Essas matrizes, apesar de cheias, podem ser representadas com complexidade linear ou logarítmico-linear devido ao comportamento dos dados nelas encontrados. Qualquer matriz pode ser aproximada por uma matriz hierárquica com uma precisão arbitrária. Porém, para a maioria das matrizes essa aproximação terá complexidade igual ou maior a da matriz original.

No caso do MEC, a suavidade assintótica das soluções fundamentais é condição suficiente para a existência da representação de baixo posto (BEBENDORF; RJASANOW, 2003 TYRTYSHNIKOV, 1996; GOREINOV; TYRTYSHNIKOV; ZAMARASHKIN, 1997; BEBENDORF, 2000). A singularidade está presente somente quando o ponto fonte coincide com o ponto campo. O tempo de montagem da matriz pode ser drasticamente reduzido usando o ACA uma vez que apenas algumas das entradas da matriz original precisam ser calculadas.

A ideia básica é dividir a matriz em blocos, baseados no tamanho e na distância entre um grupo de pontos de colocação e um grupo de elementos de contorno, classificando esses blocos por representações de baixo posto ou posto cheio. Para mostrar intuitivamente como são realizadas essas aproximações e os requerimentos que as possibilitam, será mostrado um exemplo simples como em Kurz, Rain e Rjasanow (2007).

Considere $K:[0,1] \times[0,1] \rightarrow \mathbb{R}$ uma função de dois escalares e $\mathbf{A} \in \mathbb{R}^{n \times n}$ uma matriz cujos elementos $a_{k l}$ são dados por:

$$
a_{k l}=K\left(x_{k}, y_{l}\right), k=1, \ldots, n, l=1, \ldots, n,
$$

com $\left(x_{k}, y_{l}\right) \in[0,1] \times[0,1]$. Fica evidente que a memória necessária para armazenar essa matriz é de ordem $n^{2}$ e a quantidade de operações necessárias para realizar uma multiplicação matriz-vetor também é $n^{2}$. O crescimento quadrático dessas grandezas torna os valores proibitivos mesmo para um $n$ não muito grande. A alternativa proposta é procurar uma aproximação $\tilde{\mathbf{A}}$ da matriz $\mathbf{A}$ diminuindo a memória ocupada e realizar as operações com a aproximação no lugar da matriz original.

Para garantir a qualidade dessa aproximação, define-se uma tolerância $\varepsilon$, tal que: 


$$
\|\mathbf{A}-\tilde{\mathbf{A}}\|_{f} \leq \varepsilon\|\mathbf{A}\|_{f}
$$

onde $\|\mathbf{A}\|_{f}$ é a norma de Frobenius da matriz $\mathbf{A}$, dada por:

$$
\|\mathbf{A}\|_{f}=\left(\sum_{k, l} a_{k l}^{2}\right)^{1 / 2}
$$

A escolha de valores menores de $\varepsilon$ melhoram a aproximação enquanto aumentam o custo computacional.

A melhor aproximação que pode ser obtida para a matriz A é calculada a partir da sua decomposição em valores singulares. Essa decomposição reescreve a matriz pelo produto

$$
\mathbf{A}=\mathbf{U S V}^{t}
$$

onde $\mathbf{S}$ é uma matriz diagonal $n$ por $n$ cujos elementos são seus valores singulares e as colunas de $\mathbf{U}$ e $\mathbf{V}$ são os vetores singulares associados da matriz A. A aproximação procurada descarta os menores valores singulares e seus vetores associados enquanto a equação (3.2) estiver sendo obedecida. Essa decomposição não é usada normalmente, uma vez que ela apresenta elevado custo: são necessárias $n^{3}$ operações. Ela será usada aqui para ilustrar o funcionamento do método, vindo a ser substituída, posteriormente, pela ACA.

A função $K$, apresentada na equação (3.1), é definida como:

$$
K(x, y)=1 /\left[\alpha+(x-y)^{2}\right] .
$$

Desta forma, obtém-se uma matriz A que terá um comportamento semelhante ao esperado das matrizes de elementos de contorno. A função $K$ tem uma quase singularidade que pode ser controlada pelo parâmetro $\alpha$, uma vez que ao se reduzir o valor desse parâmetro a singularidade fica mais evidente. Esse comportamento pode ser observado nas figuras 3.1, 3.2, 3.3 e 3.4 onde é apresentada a função $K$ para distintos valores de $\alpha$

O domínio será divido em ambas direções em $n$ partes iguais. A matriz A é obtida para diferentes valores de $\alpha, 10^{0}, 10^{-1}, 10^{-2}$ e $10^{-3}$, e de $n, 100,200,400$ e 800.

As figuras 3.5, 3.6, 3.7 e 3.8 mostram a evolução da aproximação para quantidades diferentes de entradas. Por entradas, entende-se a quantidade de valores singulares utilizados na aproximação. Como era esperado, ao utilizar mais valores singulares obtém-se uma aproximação melhor e consequentemente um menor erro. Porém, é interessante observar que o comportamento se mantém o mesmo para diferentes valores de 


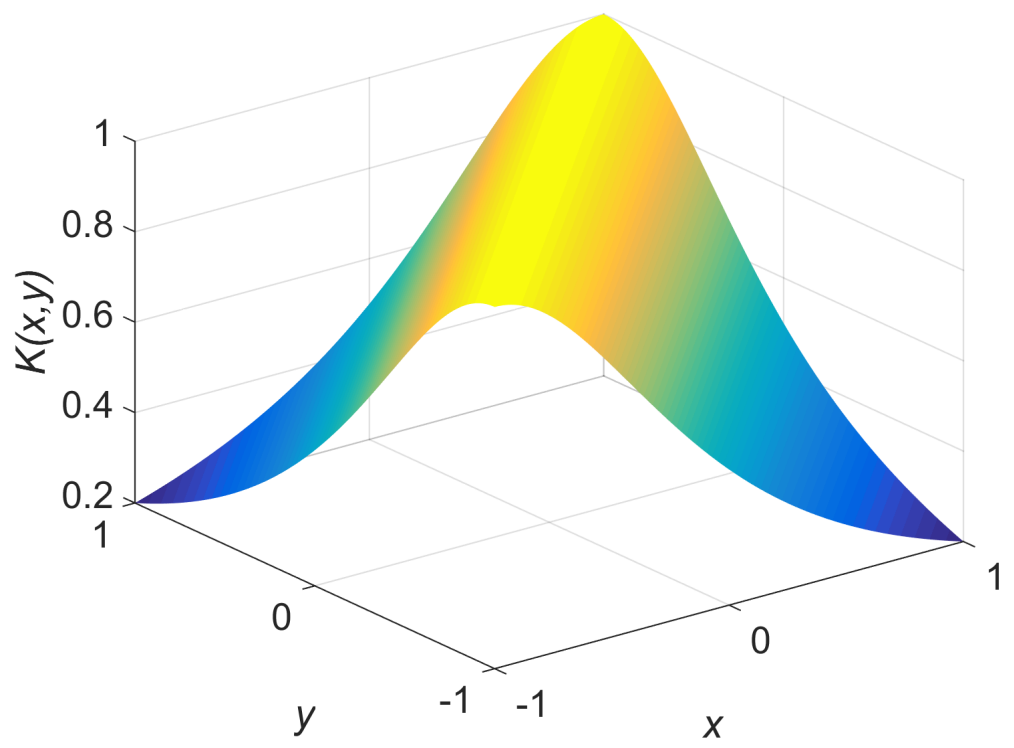

Figura 3.1: Função $K$ para $\alpha=10^{0}$.

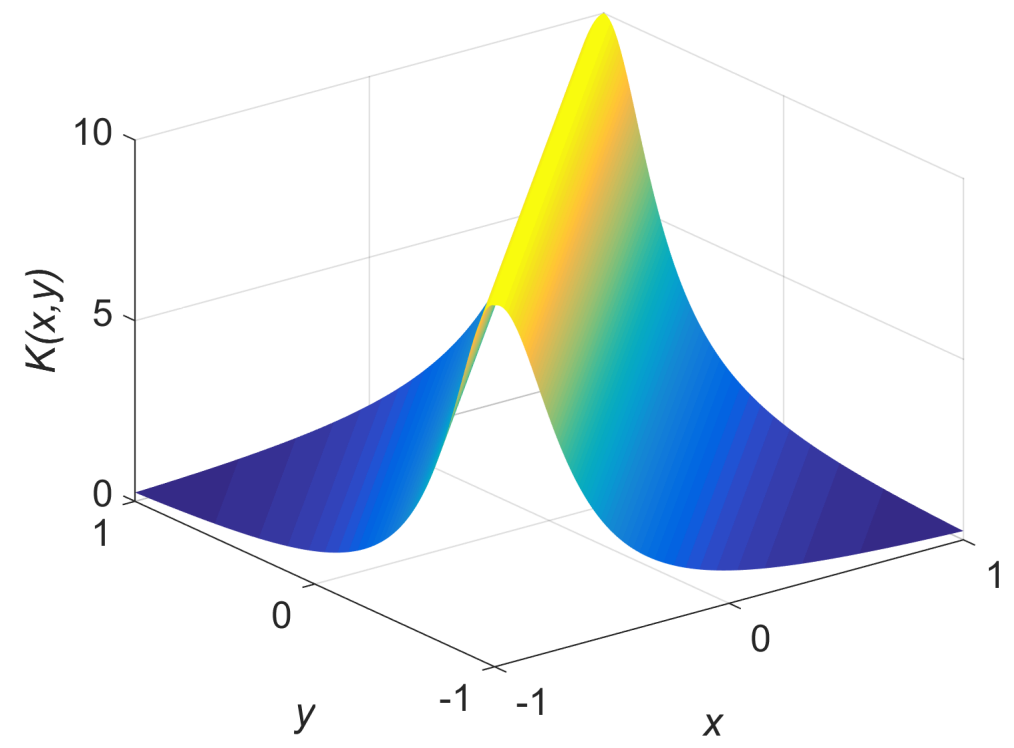

Figura 3.2: Função $K$ para $\alpha=10^{-1}$. 


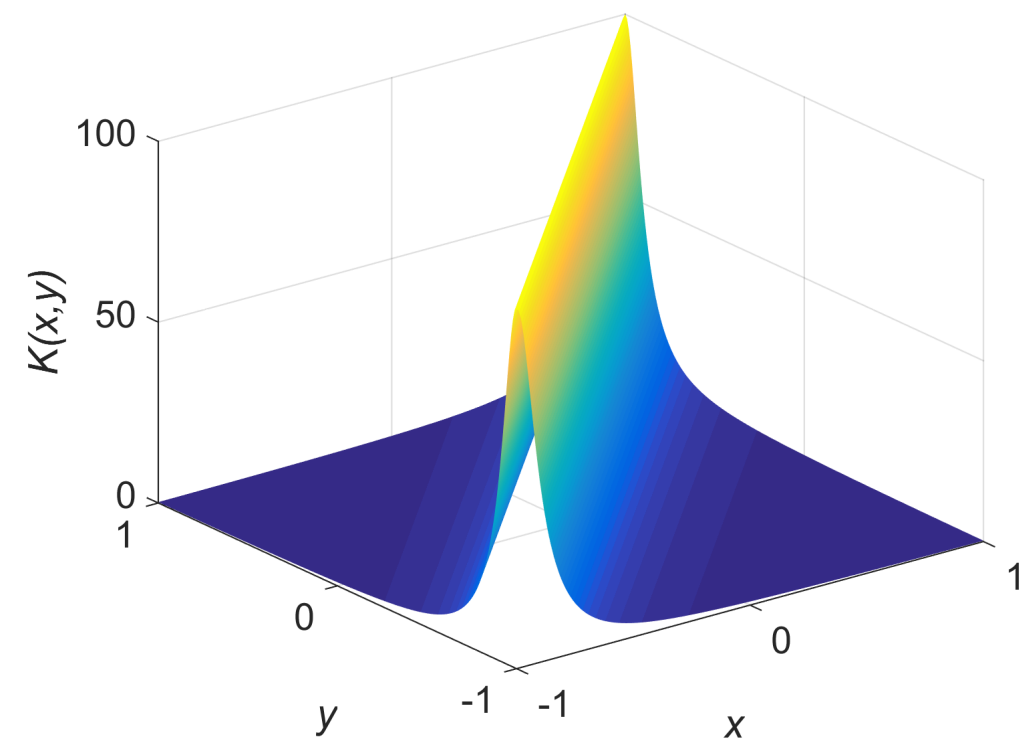

Figura 3.3: Função $K$ para $\alpha=10^{-2}$.

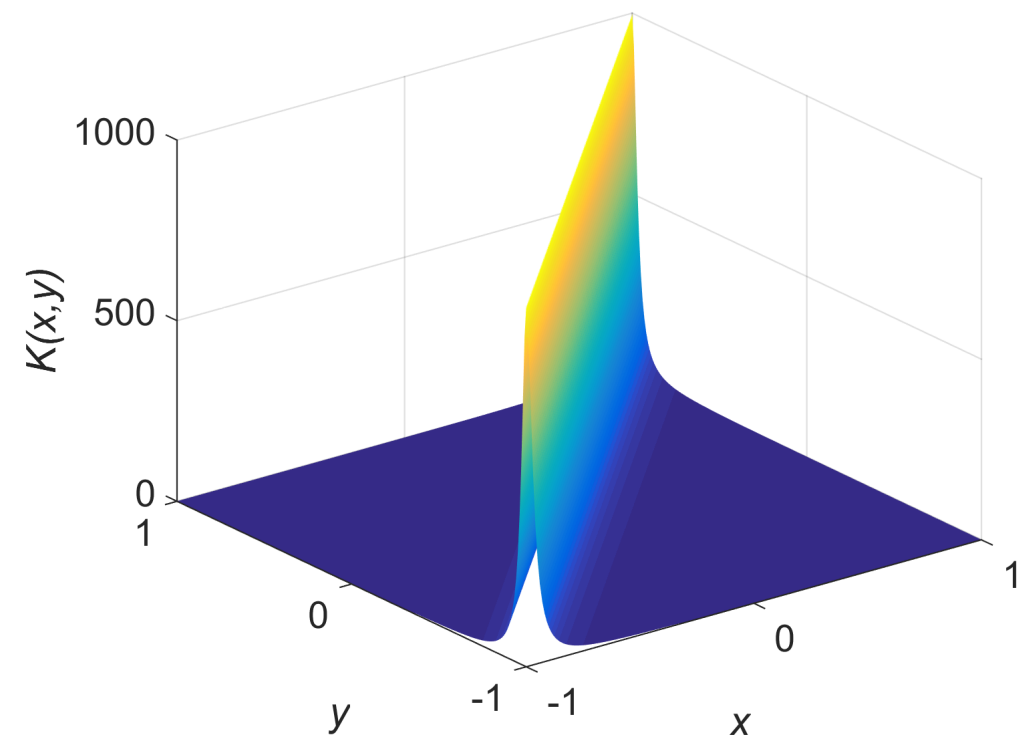

Figura 3.4: Função $K$ para $\alpha=10^{-3}$. 
$n$. Isso é o que torna o método eficiente para problemas de larga escala. A aproximação precisa de $n(2 m+1)$ entradas, onde $m$ é o número de valores singulares, para aproximar uma matriz de tamanho $n$, que necessitaria de $n^{2}$ entradas. E como o parâmetro $m$ se mantém constante, o número de entradas vai apresentar um crescimento linear onde antes era quadrático.

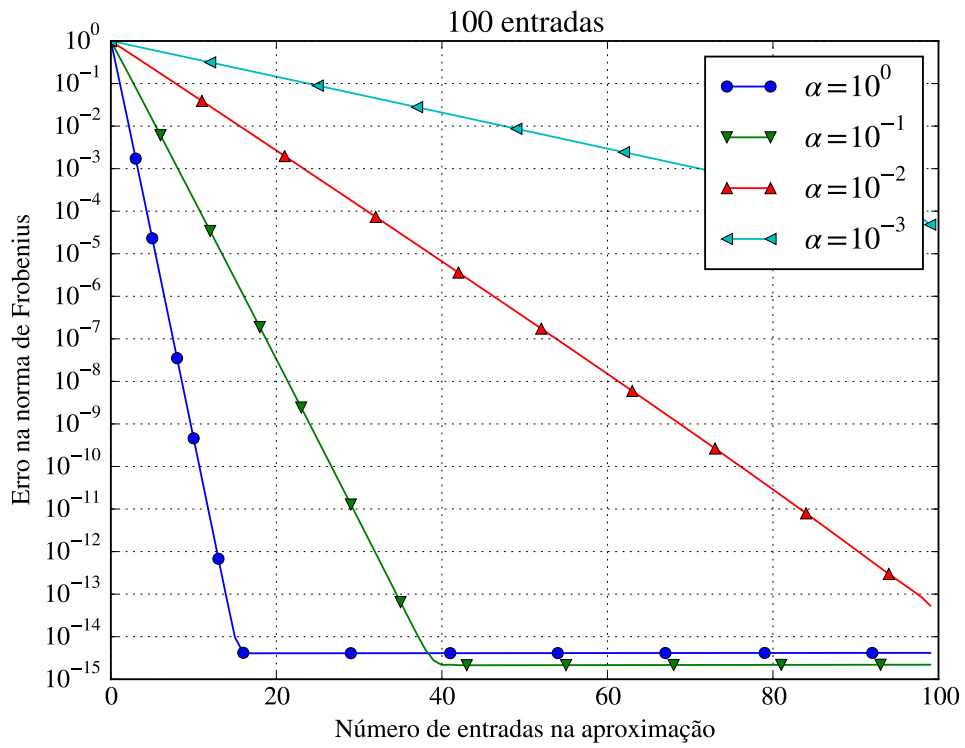

Figura 3.5: Erro na norma de Frobenius para a aproximação com SVD para $n=100$.

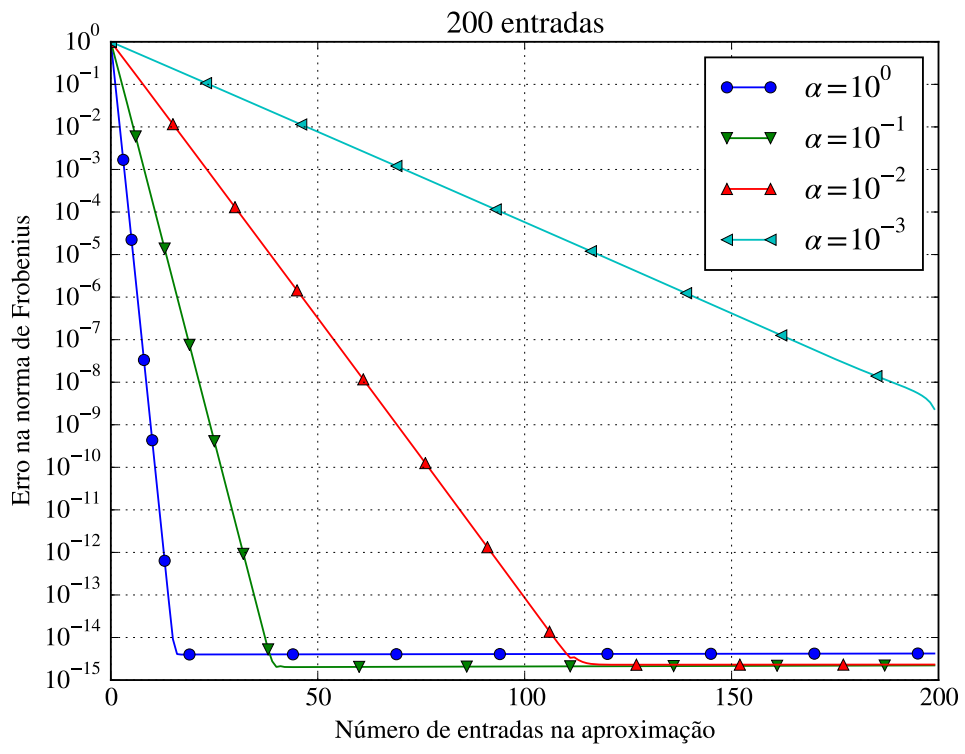

Figura 3.6: Erro na norma de Frobenius para a aproximação com SVD para $n=200$. 


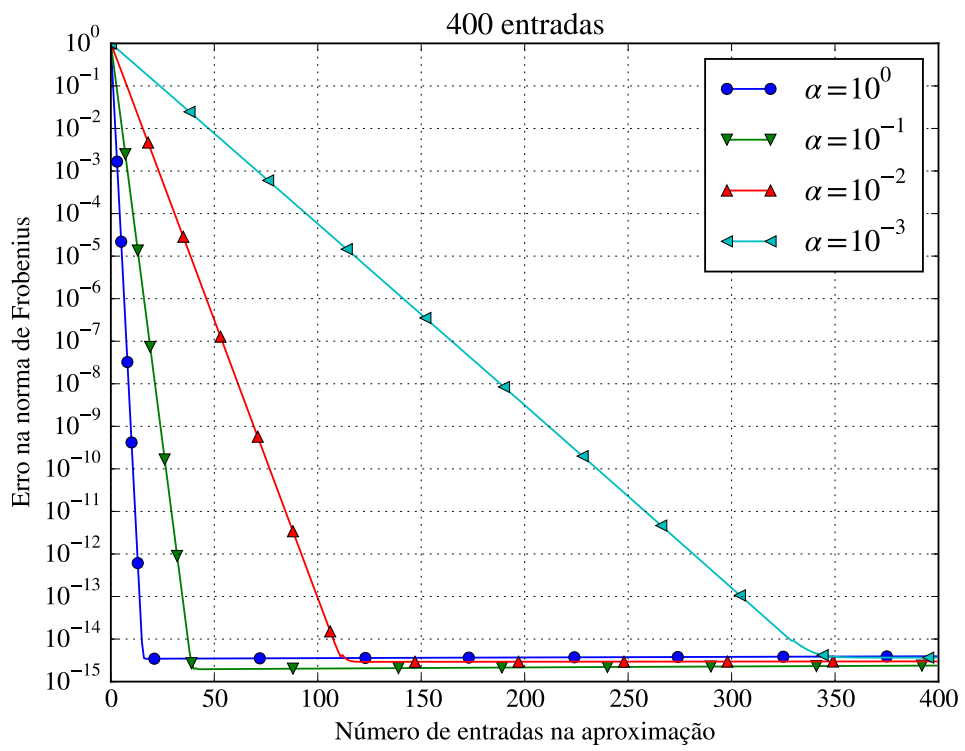

Figura 3.7: Erro na norma de Frobenius para a aproximação com SVD para $n=400$.

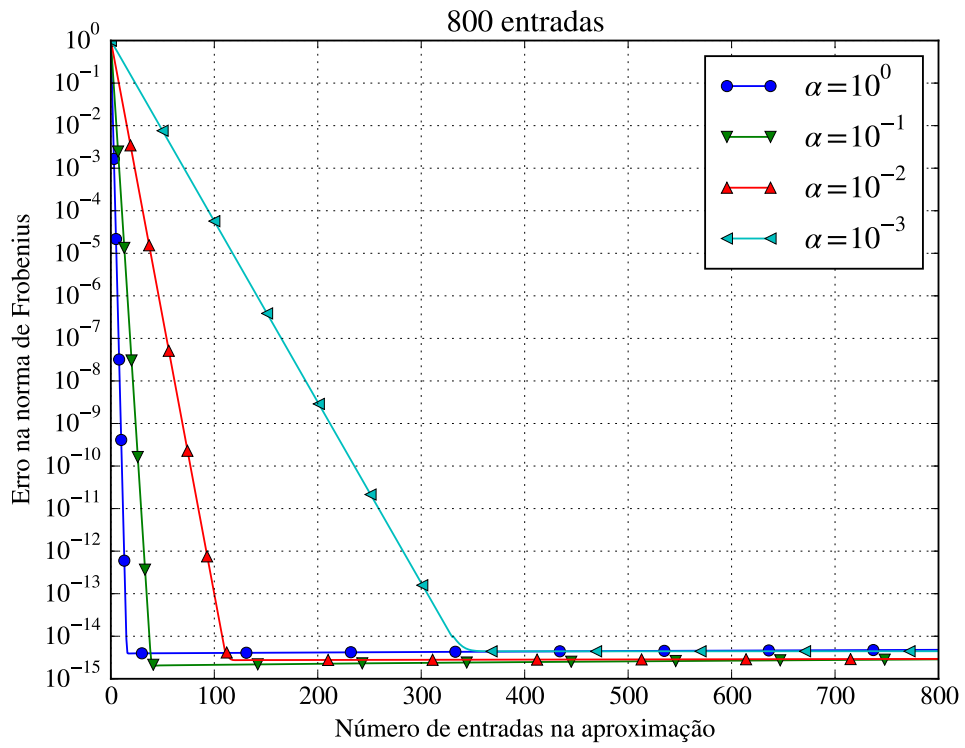

Figura 3.8: Erro na norma de Frobenius para a aproximação com SVD para $n=800$. 
A tabela 3.1 mostra o valor de $m$ para que se obtenha um $\varepsilon$ menor que $10^{-6}$ e a razão entre a memória gasta pela aproximação e a gasta pela matriz original. Nessa tabela fica evidente a economia de memória proporcionada por essa aproximação. Nos casos onde a singularidade é mais fraca, observa-se que mesmo para matrizes pequenas a aproximação é vantajosa. No entanto, quando a singularidade é aumentada, esse vantagem só é observada para matrizes maiores, não sendo possível obter uma representação com o erro requerido para $n=100$ e $\alpha=10^{-3}$.

Tabela 3.1: Memória gasta na aproximação com SVD.

\begin{tabular}{|c|c|c|c|c|c|}
\hline$\alpha$ & $m$ & $n=100$ & $n=200$ & $n=400$ & $n=800$ \\
\hline $10^{0}$ & 7 & $15,000 \%$ & $7,500 \%$ & $3,750 \%$ & $1,875 \%$ \\
\hline $10^{-1}$ & 16 & $33,000 \%$ & $16,500 \%$ & $8,250 \%$ & $4,125 \%$ \\
\hline $10^{-2}$ & 47 & $95,000 \%$ & $47,500 \%$ & $23,750 \%$ & $11,875 \%$ \\
\hline $10^{-3}$ & 142 & não convergiu & $142,500 \%$ & $71,250 \%$ & $35,625 \%$ \\
\hline
\end{tabular}

O próximo passo é subdividir a matriz $\mathbf{A}$ na tentativa de isolar os efeitos da singularidade. Na figura 3.9 pode-se observar a divisão da matriz A em 4 partes em cada direção. Essa divisão gera três diferentes regiões com distintos comportamentos, de acordo com a proximidade da diagonal principal e, por consequência, da singularidade. A tabela (3.2) contém os valores de $m$ para diferentes graus de singularidade e com um $\varepsilon$ menor que $10^{-6}$.

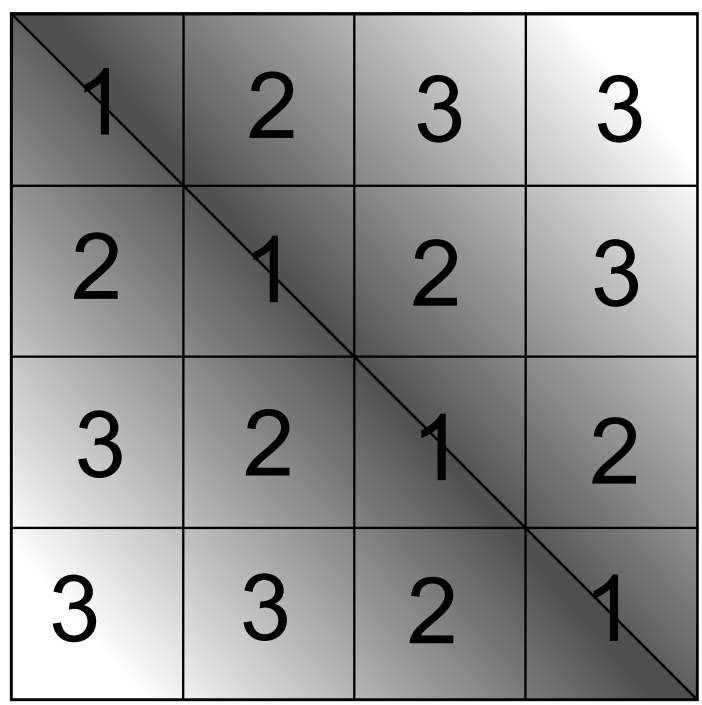

Figura 3.9: Divisão em blocos da matriz

Agora pode-se observar claramente o efeito da singularidade na aproximação, os blocos que estão ao longo da diagonal apresentam os maiores valores de $m$. Para valores suficientemente grandes de $m$, trabalhar com a aproximação se torna mais caro que do que com a matriz original. Na tabela 3.3 estão os novos valores para as razões entre as 
Tabela 3.2: Valores de $m$ para as diferentes áreas da figura 3.9 .

\begin{tabular}{|c|c|c|c|c|}
\hline Área & $\alpha=10^{0}$ & $\alpha=10^{-1}$ & $\alpha=10^{-2}$ & $\alpha=10^{-3}$ \\
\hline 1 & 3 & 6 & 14 & 37 \\
\hline 2 & 3 & 5 & 7 & 8 \\
\hline 3 & 3 & 4 & 4 & 4 \\
\hline
\end{tabular}

memórias. Ao comparar com a tabela 3.2 fica evidente, que quando a singularidade é relevante, a divisão por blocos é essencial, sendo a memória reduzida em 3 vezes para $\alpha=10^{-3}$. No entanto, para $\alpha=10^{0}$ a divisão em blocos ocupa mais memória que a aproximação da matriz inteira.

Tabela 3.3: Memória gasta na aproximação com a divisão em blocos da figura 3.9

\begin{tabular}{|c|c|c|c|c|}
\hline$\alpha$ & $n=100$ & $n=200$ & $n=400$ & $n=800$ \\
\hline $10^{0}$ & $17,50 \%$ & $8,75 \%$ & $4,38 \%$ & $2,19 \%$ \\
\hline $10^{-1}$ & $28,00 \%$ & $14,00 \%$ & $7,00 \%$ & $3,50 \%$ \\
\hline $10^{-2}$ & $47,00 \%$ & $23,50 \%$ & $11,75 \%$ & $5,88 \%$ \\
\hline $10^{-3}$ & $94,50 \%$ & $47,25 \%$ & $23,63 \%$ & $11,81 \%$ \\
\hline
\end{tabular}

Na figura 3.10 observa-se uma nova proposta na divisão por blocos da matriz. Essa divisão só ocorre nos blocos que apresentaram um valor elevado de $m$, visto que somente nesses blocos essa redivisão é vantajosa, como pôde ser observado na comparação entre as tabelas 3.2 e 3.3 para $\alpha=10^{\circ}$.

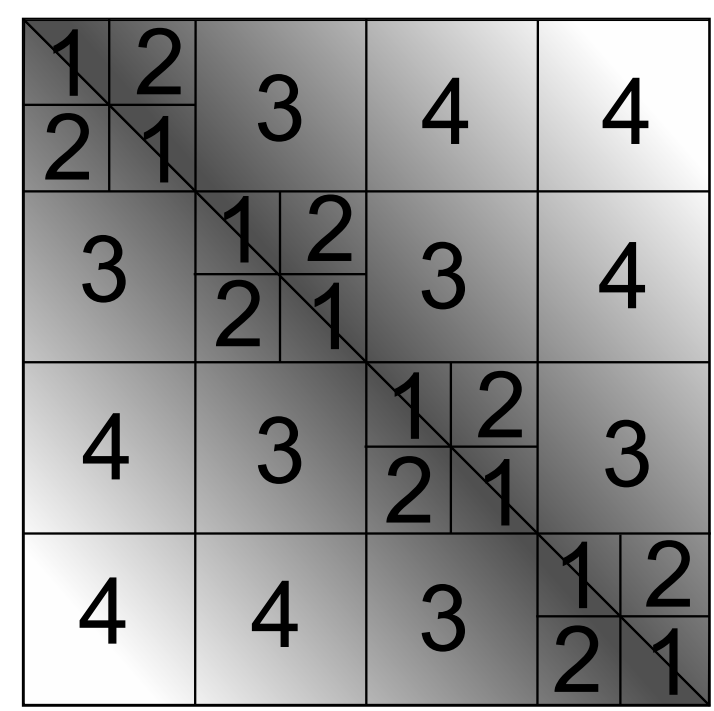

Figura 3.10: Redivisão em blocos da matriz

$\mathrm{Na}$ tabela 3.4 estão listados os valores de $m$ encontrados para as diferentes áreas da figura 3.10. A redivisão se mostra eficaz em reduzir o valor de $m$ para $\alpha=10^{-2}$ e $\alpha=10^{-3}$. Essa redução resultará em uma significativa economia de memória como pode ser observado na tabela 3.5 . 
Tabela 3.4: Valores de $m$ para as diferentes áreas da figura 3.10 .

\begin{tabular}{|c|c|c|c|c|}
\hline Área & $\alpha=10^{0}$ & $\alpha=10^{-1}$ & $\alpha=10^{-2}$ & $\alpha=10^{-3}$ \\
\hline 1 & 3 & 4 & 8 & 19 \\
\hline 2 & 3 & 4 & 6 & 7 \\
\hline 3 & 3 & 5 & 7 & 8 \\
\hline 4 & 3 & 4 & 4 & 4 \\
\hline
\end{tabular}

Tabela 3.5: Memória gasta na aproximação com a divisão em blocos da figura 3.10

\begin{tabular}{|c|c|c|c|c|}
\hline$\alpha$ & $n=100$ & $n=200$ & $n=400$ & $n=800$ \\
\hline $10^{0}$ & $16,63 \%$ & $8,31 \%$ & $4,16 \%$ & $2,08 \%$ \\
\hline $10^{-1}$ & $22,88 \%$ & $11,44 \%$ & $5,72 \%$ & $2,86 \%$ \\
\hline $10^{-2}$ & $31,38 \%$ & $15,69 \%$ & $7,84 \%$ & $3,92 \%$ \\
\hline $10^{-3}$ & $44,63 \%$ & $22,31 \%$ & $11,16 \%$ & $5,58 \%$ \\
\hline
\end{tabular}

Esse exemplo ilustra bem o poder do método para matrizes com singularidades na diagonal. As comparações dessa seção foram feitas em relação a memória gasta, mas o custo de operação, utilizando a aproximação e a matriz padrão, pode ser analisado da mesma maneira e os resultados obtidos serão similares aos apresentados.

No entanto, o exemplo trabalha com uma distribuição uniforme de pontos. Se considerarmos $x$ e $y$ como pontos fonte e campo, respectivamente, este exemplo corresponde a uma malha retilínea de pontos (problema unidimensional). Essa distribuição não é a esperada no MEC visto que somente o contorno é discretizado e este contorno estará em mais de uma dimensão podendo, inclusive, ser curvo. Além disso, a matriz foi decomposta em seus valores singulares (singular value decomposition - SVD) e a decomposição SVD não é uma técnica viável para problemas reais devido ao seu alto custo de processamento. Nas próximas seções serão apresentados a Aproximação Cruzada Adaptativa (ACA) como alternativa à decomposição SVD e um método de clusterização hierárquica para tratar uma distribuição qualquer de pontos.

\subsection{Matrizes de baixo posto}

O posto de uma matriz A é o número de linhas ou colunas linearmente independentes de $\mathbf{A}$. Uma matriz $\mathbf{A} \in \mathbb{R}^{n \times m}$ de posto $k$ pode ser escrita por um produto diádico:

$$
\mathbf{A}=\mathbf{U V}
$$

onde $\mathbf{U} \in \mathbb{R}^{n \times k} \mathrm{e} \mathbf{V} \in \mathbb{R}^{k \times m}$. Essa representação é exata e precisa de $k(m+n)$ entradas em vez de $m n$. Na figura 3.11 pode-se observar essa representação num caso onde ela é vantajosa. 


$$
\left[\begin{array}{ll}
x & x \\
x & x \\
x & x \\
x & x \\
x & x \\
x & x \\
x & x
\end{array}\right]_{\mathrm{n} \times \mathrm{k}}\left[\begin{array}{lllllll}
x & x & x & x & x & x & x \\
x & x & x & x & x & x & x
\end{array}\right]=\left[\begin{array}{lllllll}
x & x & x & x & x & x & x \\
x & x & x & x & x & x & x \\
x & x & x & x & x & x & x \\
x & x & x & x & x & x & x \\
x & x & x & x & x & x & x \\
x & x & x & x & x & x & x \\
x & x & x & x & x & x & x
\end{array}\right]_{\mathrm{n} \times \mathrm{m}}
$$

Figura 3.11: Aproximação de baixo posto: algumas entradas da matriz original representam o bloco inteiro.

Outra vantagem é quando se faz uma multiplicação matriz-vetor (HACKBUSCH; NOWAK, 1989):

$$
\mathbf{A x}=\mathbf{U V x}=\mathbf{U}(\mathbf{V} \mathbf{x})
$$

Agora, em vez de serem necessárias $2 m n$ operações aritméticas, são necessárias $2 k(m+$ $n)-k$ operações para realizar essa multiplicação. Para baixos valores de $k$, essa representação será vantajosa. Porém, ao se considerar uma matriz de posto completo $(k=n)$, observa-se que a representação por um produto diádico ocupará o dobro de memória. Portanto, a matriz $A$ é considerada de baixo posto quando:

$$
k(m+n)<m n .
$$

Essa é condição suficiente para que essa representação seja vantajosa. Matrizes que tem um posto muito menor que as suas dimensões são a base da eficiência das matrizes hierárquicas.

\subsubsection{Aproximação Cruzada}

Goreinov, Tyrtyshnikov e Zamarashkin (1997) apresentaram uma alternativa à decomposição SVD sobre o nome de aproximação esqueleto. O método consiste em dois passos: 
- Encontra-se o par de índices $\left(i^{*}, j^{*}\right)$ de maior valor, em módulo, da matriz.

- calcula-se os vetores $U_{i, 1}=A_{i, j^{*}}$ e $V_{1, j}=A_{i^{*}, j} / A_{i^{*}, j^{*}}$.

A matriz $\tilde{\mathbf{A}}=\mathbf{U V}$ é a aproximação de posto 1 que era procurada. Para encontrar uma aproximação de posto $k$ deve-se repetir esses dois passos $k$ vezes ao resto $\mathbf{A}-\tilde{\mathbf{A}}$ acrescentando, a cada passo, uma coluna à matriz $\mathbf{U}$ e uma linha a matriz V. Essa aproximação representa exatamente uma matriz de posto $k$ em $k$ passos.

Uma alternativa é usar um pivotamento parcial onde não é necessário conhecer a matriz inteira previamente nem procurar os índices $\left(i^{*}, j^{*}\right)$ em toda a matriz. A escolha dos índices é feita ao longo de um índice fixo, ou seja, o elemento máximo é obtido em apenas uma coluna ou linha.

O algorítimo 3.1 mostra o procedimento completo para a aproximação cruzada parcialmente pivotada. Nele o operador : representa todas as entradas naquela direção e max uma função que retorna o valor máximo e a posição no vetor desse valor. A condição abs $\left(a_{\max }\right)<10^{-12}$ apresenta um problema para matriz esparsas, como as posições não-nulas não são previamente conhecidas, o método é obrigado a encontrar todas essas posições, o que torna essa técnica inutilizável nesses tipos de matrizes. Entretanto, para a maioria das matrizes do MEC o método pode ser utilizado com sucesso pois as mesmas são, quase sempre, cheias. A matriz $\mathbf{H}$, por possuir na sua formulação um produto escalar entre dois vetores, terá blocos de zeros quando estes vetores forem ortogonais. Este problema será tratado mais adiante nesse capítulo.

\subsubsection{Aproximação Cruzada Adaptativa}

Outra alternativa é a aproximação cruzada adaptativa. Essa variação determina o posto $k$ adaptativamente com uma precisão $\varepsilon$. Uma boa heurística é estimar o erro $\left\|\mathbf{A}-\mathbf{A}^{\prime}\right\|$ por uma aproximação de posto um. Em cada passo é justamente uma aproximação de posto um que é calculada, portanto pode-se estimar que:

$$
\left\|\mathbf{A}-\mathbf{A}^{\prime k}\right\| \lesssim\left\|\mathbf{A}-\mathbf{A}^{\prime k-1}\right\| \approx\left\|\mathbf{A}^{\prime k}-\mathbf{A}^{\prime k-1}\right\|=\left\|\mathbf{a}_{n} \mathbf{b}_{n}\right\|
$$

onde $\mathbf{a}_{n}$ e $\mathbf{b}_{n}$ são o vetor e coluna gerados no passo $n$ do método.

Portanto, o erro relativo, utilizado no algorítimo, pode ser estimado usando a norma euclidiana ou de Frobenius como:

$$
\epsilon_{\text {rel }}=\frac{\left\|\mathbf{a}_{n} \mathbf{b}_{n}\right\|}{\left\|\mathbf{A}^{\prime 1}\right\|}
$$

Usando o algorítimo 3.2 , a matriz a ser aproximada não precisa ser calculada previamente: somente as colunas e linhas necessárias para a aproximação serão calculadas. 


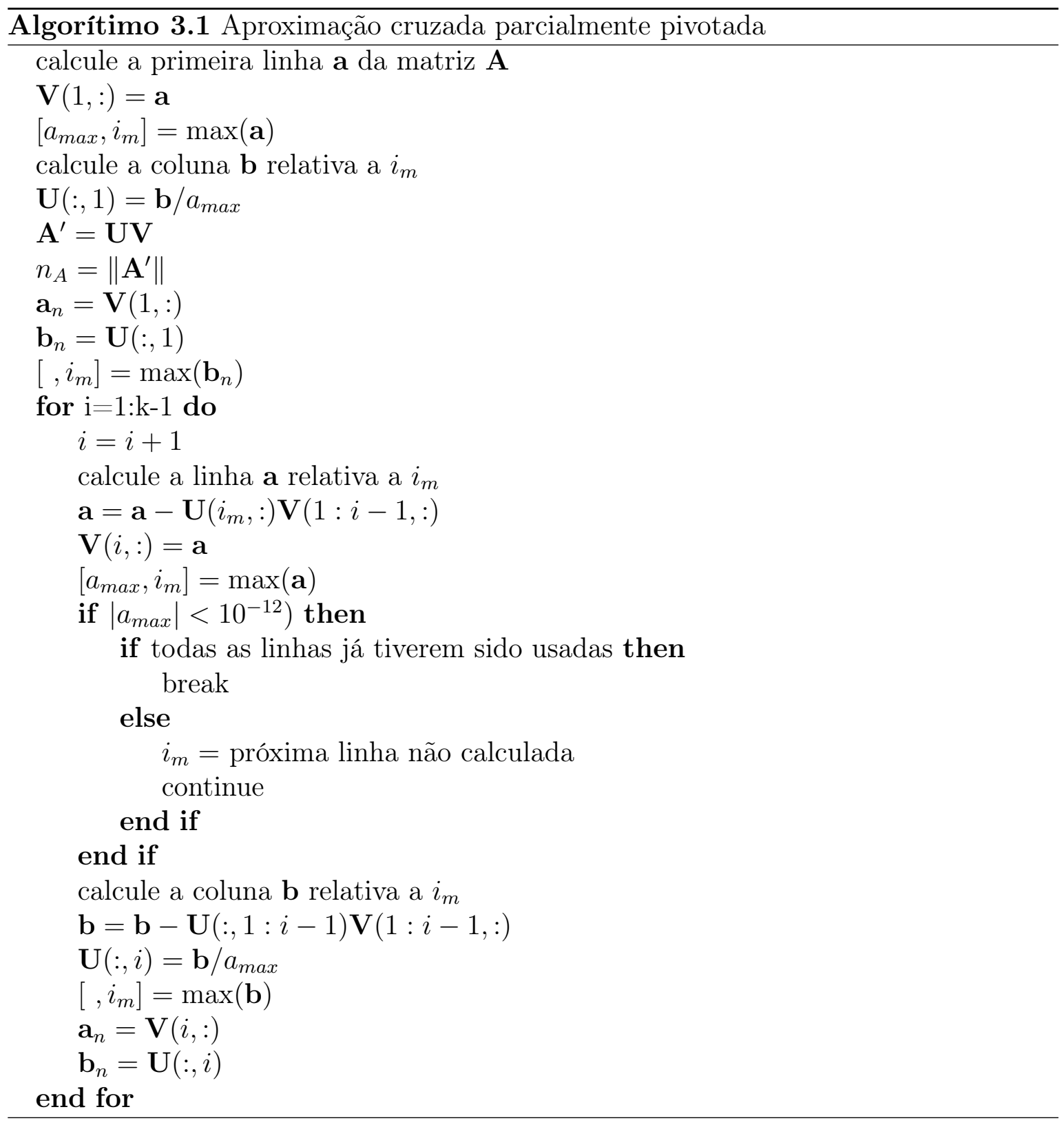


Tanto os requerimentos de memória quanto o número de operações são de ordem linear nesse algorítimo.

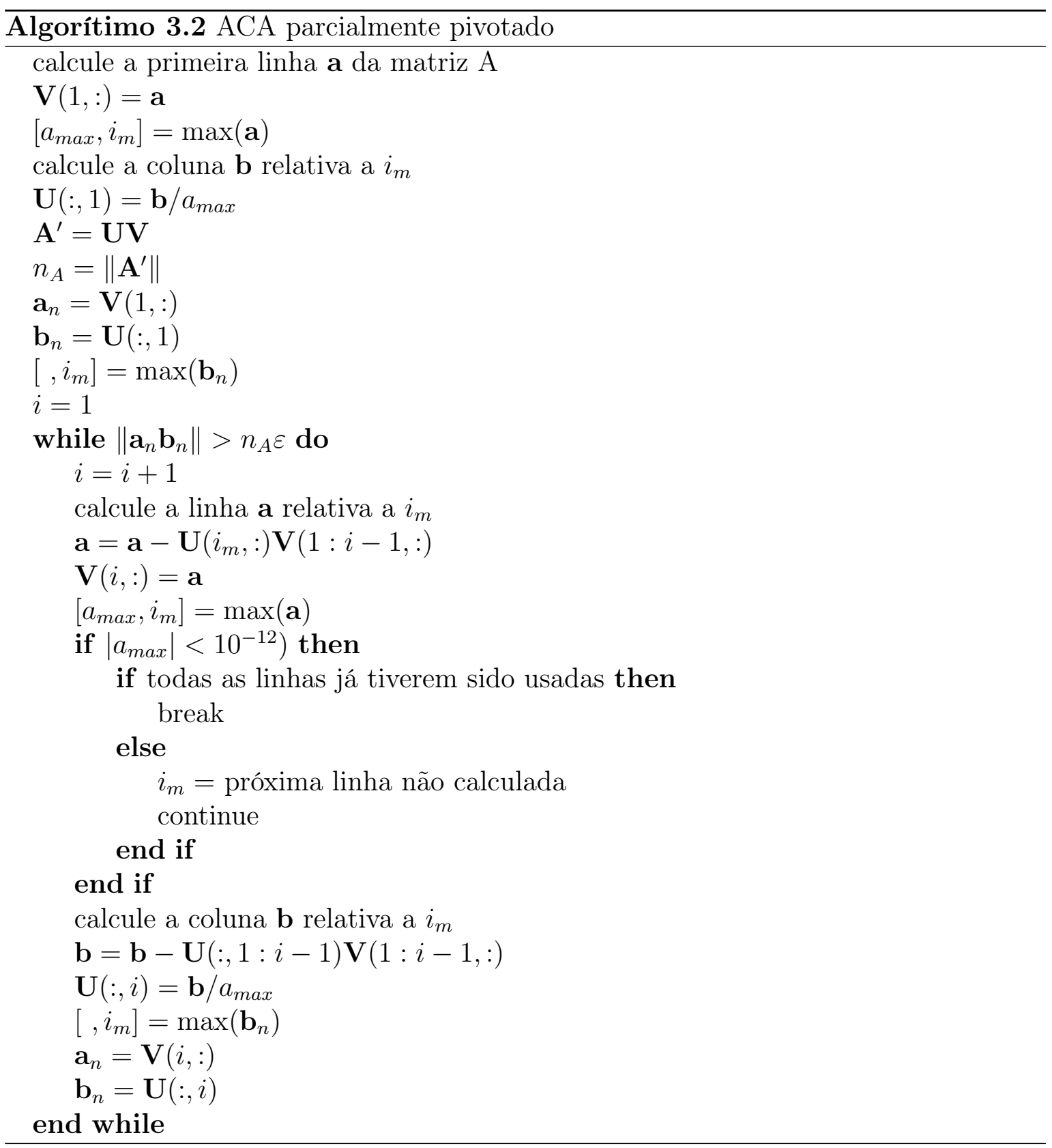

Em Bebendorf (2000), Bebendorf e Rjasanow (2003) encontra-se provas da convergência do ACA para o caso onde a matriz tenha como origem funções suaves em pontos do domínio. A hipótese de distribuição dos pontos não leva em consideração que no MEC a discretização está contida apenas no contorno. Esse detalhe pode vir a causar problemas na convergência do método como pode ser mostrado no exemplo a seguir.

Na figura 3.12 observa-se quatro distintas áreas ao longo de um canto e o bloco criado com as áreas 1 e 2 como pontos fonte e as áreas 3 e 4 como pontos campo. No caso onde a solução fundamental possui um produto interno do vetor raio com o vetor normal (matriz $\mathbf{H}$ ), a matriz gerada tem valores iguais a zero para todas as entradas 

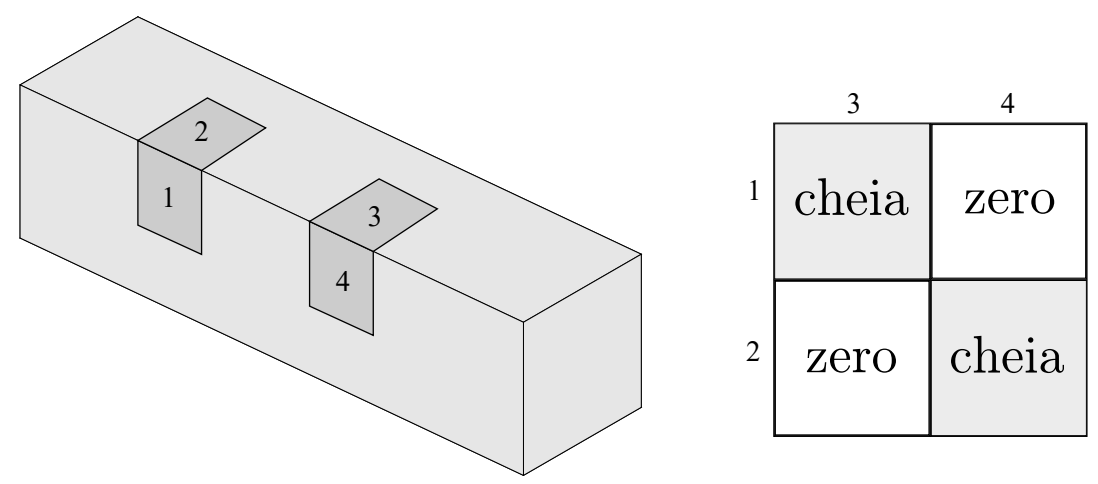

Figura 3.12: Configuração que causa problemas no ACA e a matriz resultante

onde a normal é perpendicular ao vetor raio.

Considere o uso do algorítimo 3.2 nessa situação começando com um índice pertencente a área 1. Logo todas as entradas relativas a área 4 serão iguais a 0 pois os vetores raio (que liga o ponto fonte ao ponto de integração) e a normal à superfície são ortogonais. Portanto, o máximo dessa linha estará na área 3 e o índice da coluna também. O produto interno terá entradas não nulas somente quando essas pertencerem as áreas 1 e 3. Esse comportamento se repete nos passos seguintes fazendo com que o método não enxergue o segundo bloco com as áreas 2 e 4 . Consequentemente, o algorítimo converge para uma aproximação com erro alto cujo o estimador de erro usado tem valor abaixo do procurado.

\subsubsection{Aproximação Cruzada Adaptativa modificada}

Na seção anterior observou-se, por meio de um contra-exemplo, que a utilização da ACA na montagem de matrizes de influência do MEC não é aconselhável.

Uma grande vantagem da ACA é ela poder funcionar em modo caixa-preta. Uma vez conhecida a localização das funções de base, o procedimento de montagem das matrizes não precisa ser conhecido. Em Börm e Grasedyck (2005) observa-se uma alternativa interessante que utiliza um processo interpolador criando uma abordagem híbrida. Apesar de não apresentar os problemas de convergência da ACA, essa abordagem perde a grande vantagem de poder trabalhar em modo caixa-preta.

Uma variação da $\mathrm{ACA}$, conhecida como $\mathrm{ACA}+$ e apresentada primeiramente em Grasedyck (2005), propõe uma modificação da estratégia de pivotamento com o objetivo de superar as situações onde a ACA padrão falha.

Esse método se diferencia por utilizar colunas e linhas de referência antes de calcular as entradas que serão usadas na aproximação. A coluna de referência é escolhida contraintuitivamente pela entrada de menor valor da linha. Essa escolha garante que cada bloco não-nulo seja intersectado por uma linha ou coluna de referência. 
O pivotamento é, então, definido com base nas informações obtidas nos vetores de referência, que pode ser observado no algorítimo 3.3. Esse algorítimo produz uma aproximação de posto um. No próximo passo, os vetores de referência podem ser apenas atualizados, sendo somente necessário determinar novos quando os mesmos tiverem sido usados como os próprios vetores da aproximação.

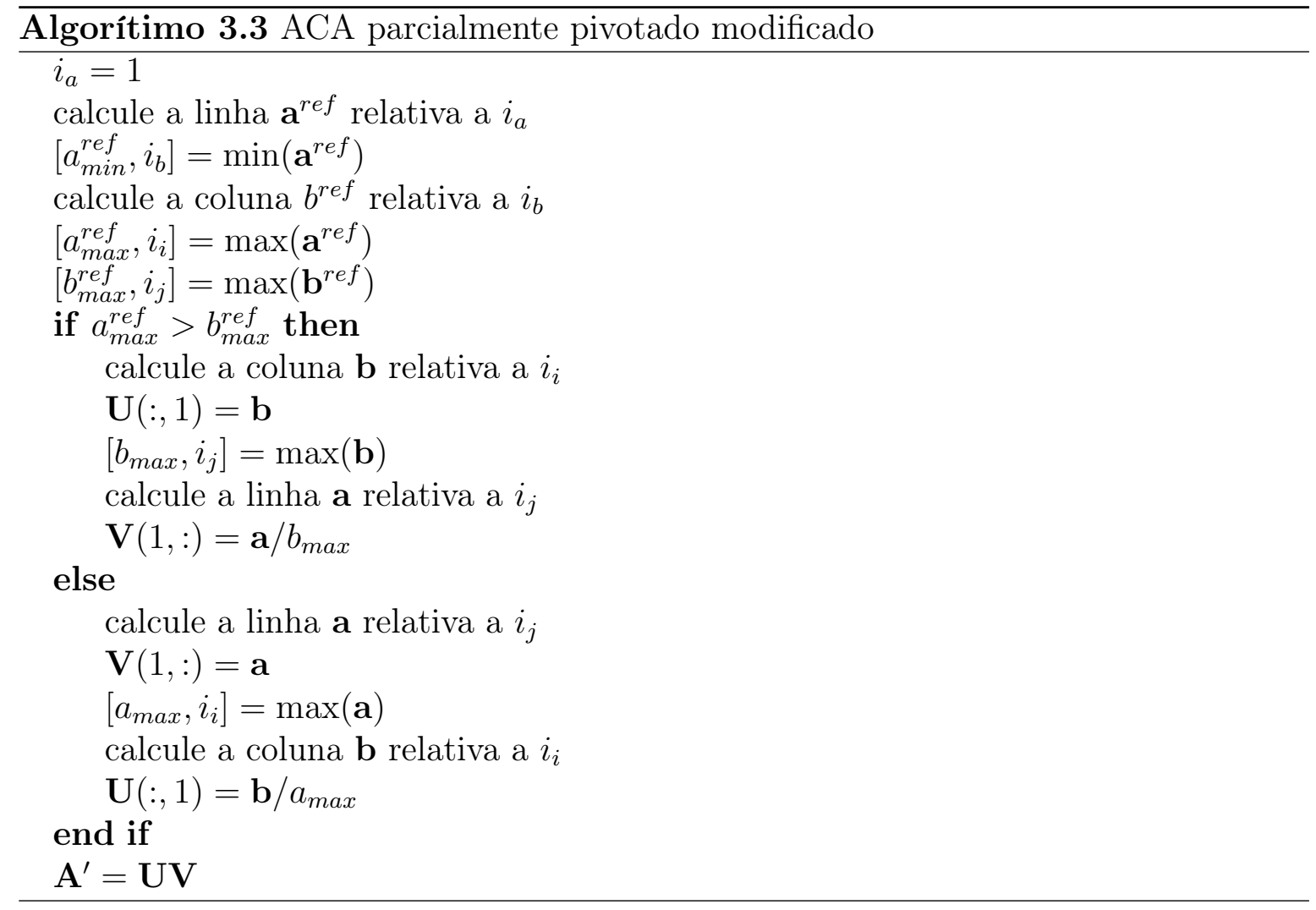

Apesar de não haver uma prova de convergência da $\mathrm{ACA}+$, ou para alguma outra técnica de pivotamento parcial, ele tem o seu funcionamento comprovado para as aplicações desse trabalho.

\subsection{Clusterização hierárquica}

Na seção anterior foi mostrado que matrizes de baixo posto podem ser representadas eficientemente por um produto diádico, com uma aproximação podendo ser obtida facilmente pela ACA. Entretanto, dificilmente se encontra matrizes globalmente de baixo posto, o que torna necessária a divisão em blocos como pôde se observar no exemplo introdutório.

Para se realizar a divisão em blocos da matriz, o primeiro passo é construir uma partição hierárquica dos pontos envolvidos na discretização. Existem diversos métodos que se propõem a realizar essa partição. Nesse trabalho será utilizada uma árvore binária. A árvore é composta de nós. O primeiro nó é conhecido como raiz e contém 
todos os pontos analisados. A partir da raiz os pontos são divididos e dois novos nós são criados. Esse processo, de divisão em dois nós, é repetido até que se atinja um número preestabelecido de pontos dentro de todos nós. O último nó da árvore, aquele que não se divide mais, é chamado de folha. Na figura 3.13 observa-se esse processo em funcionamento.

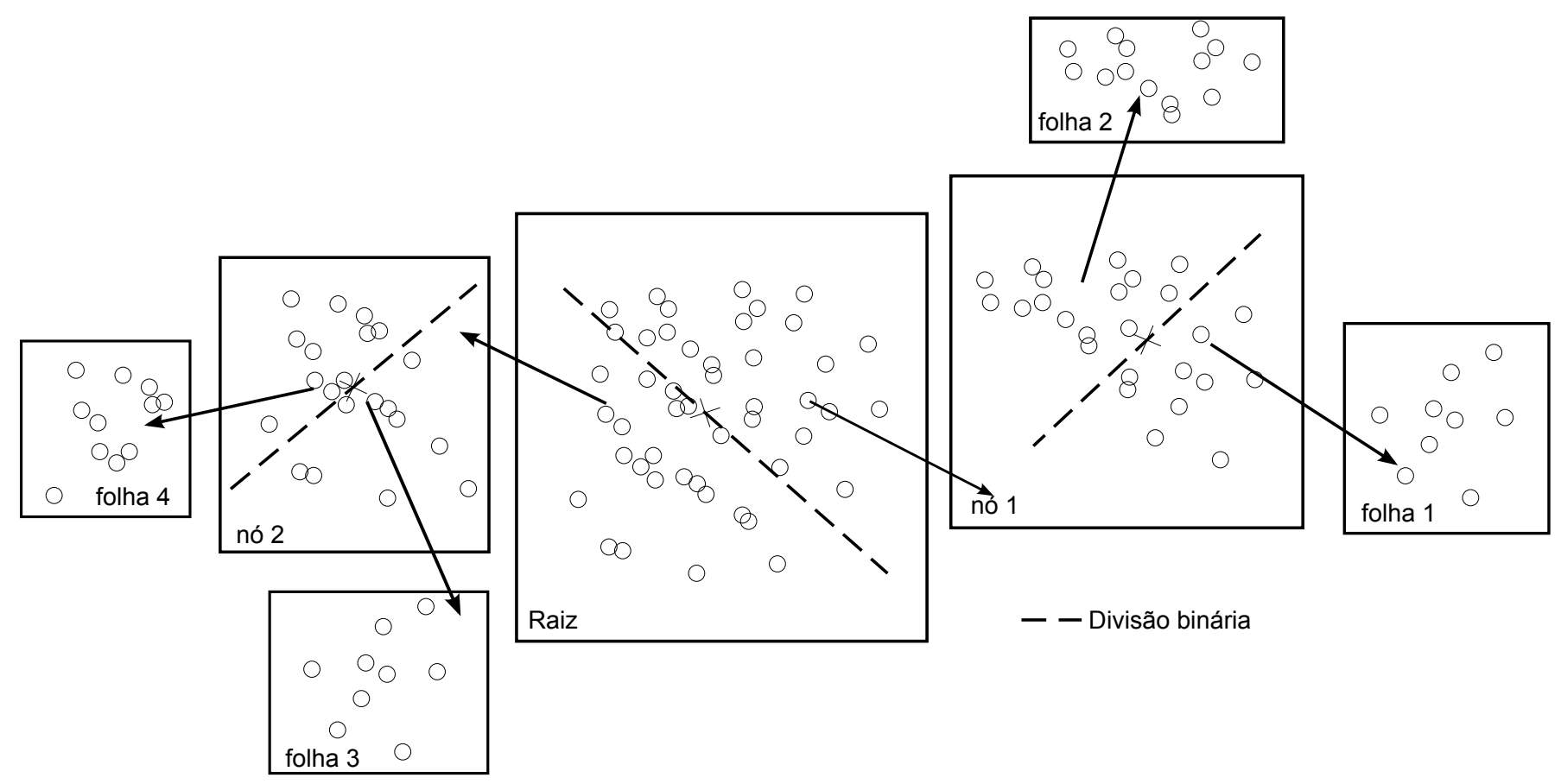

Figura 3.13: Árvore binária

Para se realizar a divisão do nó em seus filhos, o algorítimo 3.4 será utilizado.

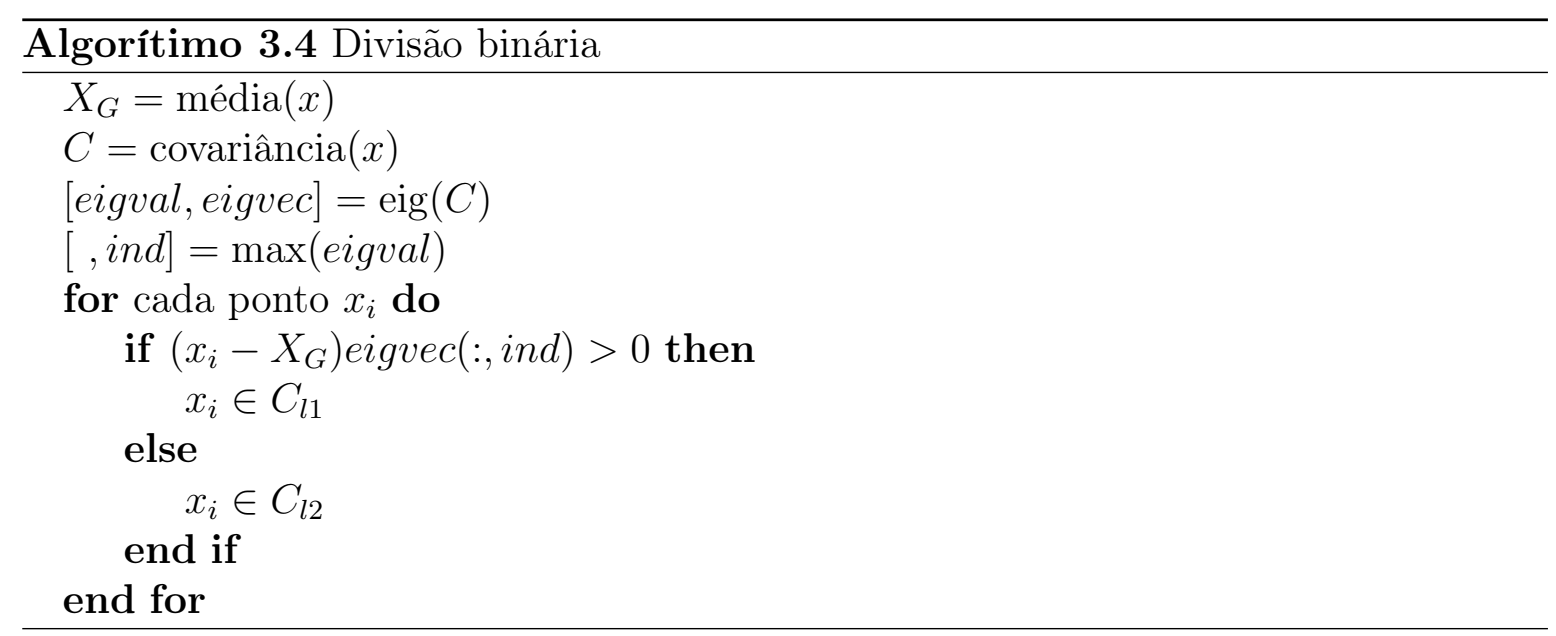

O algorítimo 3.4 usa a análise dos componentes principais (PCA) para encontrar a direção da divisão. A PCA é um método estatístico linear que encontra os autovalores e autovetores da matriz de covariância dos dados e, com esse resultado, pode-se reali- 
zar a redução dimensional dos dados e analisar os padrões principais de variabilidade presentes.

Ao se calcular o autovetor relacionado ao maior autovalor, se obtém a direção na qual o nó tem a maior variabilidade e, por consequência, a maior extensão. Ao usar $\left(x_{i}-X_{G}\right)$ eigvec $($ ind $)=0$ para separar os pontos, se garante que o plano de separação é perpendicular a esse autovetor e que passa pelo centro do nó. Ao utilizar esse algorítimo, se espera obter uma divisão mais representativa do que uma onde simplesmente se divide o nó na metade de uma das suas componentes, uma vez que a divisão se dá sempre na direção de maior extensão.

Mesmo criando a árvore, o processo de divisão em blocos não está finalizado. Cada bloco da matriz é o resultado da interação entre dois nós da árvore. O algorítimo 3.5 faz o pareamento dos diferentes nós de maneira eficiente, garantindo que os maiores blocos possíveis, obedecendo a condição de admissibilidade, formem a matriz completa. Esse conjunto de blocos, compostos de matrizes aproximadas pela ACA e matrizes cheias, é chamado de matriz hierárquica.

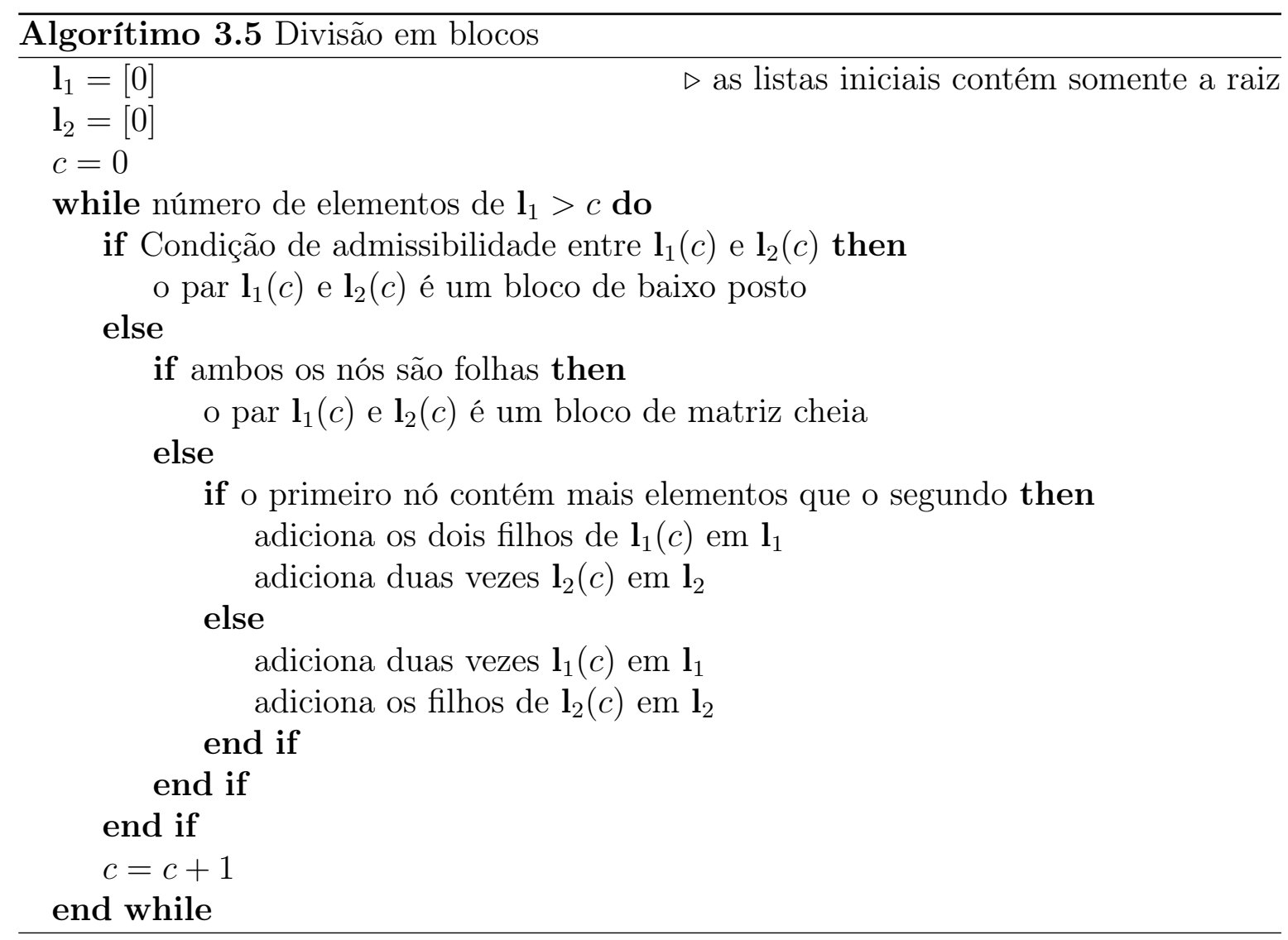

A condição de admissibilidade é o que garante a qualidade das aproximações calculadas. A ideia é assegurar que os nós de um bloco não se aproximem, de maneira a evitar que a singularidade do MEC influencie os blocos que forem aproximados. Isso certifica que as submatrizes desses blocos são de baixo posto, possibilitando, assim, sua 
aproximação. Um bloco é considerado admissível se seus nós $\mathbf{C}_{l 1}$ e $\mathbf{C}_{l 2}$ obedecerem:

$$
\min \left(\operatorname{diam}\left(\mathbf{C}_{l 1}, \mathbf{C}_{l 2}\right)\right) \leq \eta\left\|\left(\mathbf{C}_{l 1}, \mathbf{C}_{l 2}\right)\right\|
$$

onde $\eta$ é o parâmetro que define o quão separados os nós tem que estar. Com $\eta=1$ os nós poderiam se tangenciar e para $\eta=0$ a condição nunca seria atingida, pois os nós nunca estariam suficientemente afastados. Portanto, $\eta$ só pode assumir valores entre 0 e 1.

Calcular o diâmetro de um conjunto de pontos e a distância entre conjuntos resulta em um esforço computacional alto porém desnecessário uma vez que aproximações simples e ainda mais restritivas podem ser facilmente obtidas. Por exemplo, pode-se usar:

$$
\begin{aligned}
\operatorname{diam}\left(\mathbf{C}_{l 1}\right) & \leq 2 \max \left(X_{G 1}-x_{1}\right), \\
\operatorname{diam}\left(\mathbf{C}_{l 2}\right) & \leq 2 \max \left(X_{G 2}-x_{2}\right), \\
\operatorname{dist}\left(\mathbf{C}_{l 1}, \mathbf{C}_{l 2}\right) & \leq\left\|x_{1}-x_{2}\right\|-\frac{1}{2}\left(\operatorname{diam}\left(\mathbf{C}_{l 1}\right)+\operatorname{diam}\left(\mathbf{C}_{l 2}\right)\right),
\end{aligned}
$$

onde $X_{G}$ é o centro do nó calculado no algorítimo 3.4 .

\subsection{Solução do sistema linear}

Ao escrever a matriz de maneira hierárquica, não é interessante usar um método de solução direto, uma vez que ao fazê-lo não se mantém a economia de memória gerada ao utilizar essa representação. Ao usar um método iterativo, pode se fazer uso das operações matriciais aceleradas. A operação de maior relevância no contexto do MEC é multiplicação matriz-vetor. Essa operação é a base dos métodos iterativos utilizadas na solução do sistema linear, sendo ela a operação de maior custo. A representação da matriz por um produto diádico faz com que as operações matriciais tenham de ter um tratamento distinto. Grasedyck e Hackbusch (2003) e Bebendorf (2008) detalham todas as operações envolvendo as matrizes hierárquicas.

A multiplicação de uma matriz hierárquica e um vetor consiste em multiplicar cada bloco da matriz pelo subvetor correspondente às colunas daquele bloco. Se o bloco for admissível, essa multiplicação é otimizada e é calculada pela equação (3.7). Se o bloco não for admissível, essa multiplicação é calculada por meio de uma multiplicação matriz-vetor padrão. Essa é uma operação que não envolve nenhuma aproximação, obtendo o resultado exato da multiplicação. 


\subsubsection{O método dos resíduos mínimos generalizados (GMRES)}

A operação de multiplicação matriz-vetor é a que gera maior impacto na solução do sistema quando métodos iterativos são utilizados. Nesta tese, o método dos resíduos mínimos generalizados (GMRES) foi o método escolhido para a solução do sistema linear. O método, inicialmente proposto por Saad e Schultz (1986), aproxima a solução iterativamente em um subespaço de Krylov com resíduo mínimo. A iteração de Arnoldi é usada para formar a base para o subespaço de Krylov.

Considerando um sistema linear:

$$
\mathrm{Ax}=\mathrm{b}
$$

onde A é uma matriz $n \times n, \mathbf{b}$ um vetor conhecido e $\mathbf{x}$ um vetor desconhecido.

$\mathrm{O}$ procedimento se inicia com uma estimativa inicial $\mathbf{x}_{0}$ que produz um resíduo que pode ser facilmente obtido por:

$$
\mathbf{r}_{0}=\mathbf{b}-\mathbf{A x}_{0}
$$

Na iteração $m$ a solução aproximada é dada por:

$$
\mathbf{x}_{m}=\mathbf{x}_{0}+\mathbf{V}_{m} \mathbf{y}
$$

onde $\mathbf{y}$ é um vetor e $\mathbf{V}_{m}$ é uma base para o subespaço de Krylov de ordem $m$ definido como:

$$
\kappa_{m}=\left\{\mathbf{r}_{0}, \mathbf{A r}_{0}, \ldots, \mathbf{A}^{m-1} \mathbf{r}_{0}\right\}
$$

O GMRES aproxima a solução exata pelo vetor $\mathbf{x}_{m}$ que minimiza a norma do resíduo $\mathbf{r}_{m}=\mathbf{b}-\mathbf{A} \mathbf{x}_{m}$. Os vetores $\mathbf{r}_{0}, \mathbf{A r}_{0}, \ldots, \mathbf{A}^{m-1} \mathbf{r}_{0}$ são quase linearmente dependentes e, por isso, a iteração de Arnoldi é utilizada no GMRES para obter vetores ortonormais:

$$
\mathbf{v}_{1}, \mathbf{v}_{2}, \ldots, \mathbf{v}_{m}
$$

O processo de Arnoldi também produz uma matrix superior de Hessenberg, $\tilde{\mathbf{H}}_{m}$, que satisfaz a relação:

$$
\mathbf{A} \mathbf{V}_{m}=\mathbf{V}^{m+1} \tilde{\mathbf{H}}_{m}
$$

Com isso o resíduo na iteração $m$ pode ser escrito como:

$$
\mathbf{r}_{m}=\mathbf{b}-\mathbf{A X}_{m}=\mathbf{b}-\mathbf{A}\left(\mathbf{X}_{0}+\mathbf{V}_{m} Y\right)=\mathbf{r}_{0}-\mathbf{A} \mathbf{V}_{m} \mathbf{y}=\mathbf{r}_{0}-\mathbf{V}_{m+1} \tilde{\mathbf{H}}_{m} \mathbf{Y}
$$


A aproximação é então obtida encontrando um valor de y que minimize a norma do resíduo e substituindo na equação (3.15). O algorítimo GMRES tem a propriedade que a norma do resíduo pode ser computada sem que a iteração tenha sido formada, ou seja, tem-se o cálculo a priori do erro. Logo, a ação custosa de formar a iteração pode ser adiada até que a norma do resíduo seja considerada pequena o suficiente.

O GMRES padrão tem um sério problema: a quantidade de trabalho computacional e memória utilizada aumenta linearmente a cada iteração. O custo se torna rapidamente proibitivo, a não ser que a convergência seja extremamente rápida. A maneira padrão de lidar com esse problema é reiniciar a iteração. Depois de um número prédefinido de passos, os dados acumulados são eliminados e os resultados intermediários são usados como uma nova estimativa inicial. Esse procedimento é repetido até que a convergência seja alcançada. A dificuldade está em escolher um valor apropriado para quantidade de passos. Se esse número for pequeno demais, o GMRES pode convergir vagarosamente ou falhar completamente. Um valor muito grande envolve um custo elevado.

A escolha de um parâmetro de recomeço ainda é motivo de pesquisa e não se tem um consenso de como realizá-la. Em Joubert (1994), Vorst e Vuik (1993), Saad e Schultz (1986) observa-se que para valores grandes do parâmetro há uma melhora na convergência do método. No entanto, em Eiermann, Ernst e Schneider (2000), Embree (2003) ficou evidente que valores menores do parâmetro podem resultar em menos iterações para alguns problemas. Esse resultado mostra como é difícil definir um valor para esse parâmetro. Usualmente se procura escolher previamente um valor que balanceie as propriedades de boa convergência tipicamente associadas com valores grandes do parâmetro e a redução do custo computacional resultante de um valor menor. No entanto, algumas propostas vem sendo apresentadas para definir o parâmetro de recomeço adaptativamente devido a diferentes razões (BAKER; JESSUP; KOLEV, 2009, BAKER; JESSUP; MANTEUFFEL, 2005; ZHANG; NODERA, 2005; HABU; NODERA, 2000; SOSONKINA et al., 1998). Em Baker, Jessup e Kolev (2009) uma estratégia de definição do parâmetro de recomeço simples é apresentada levando em conta a natureza cíclica da direção do vetor residual. Essa técnica se mostrou consistentemente mais eficiente do que utilizar um parâmetro pré-definido e por isso foi utilizada nesse trabalho. Uma apresentação completa e detalhada do método GMRES foge do escopo dessa tese. Para um maior detalhamento sugere-se consultar Merkel et al. (1998), Leung e Walker (1997) para a formulação padrão do método e Baker, Jessup e Kolev (2009) para a modificação utilizada. 


\section{CURVAS E SUPERFÍCIES NURBS}

\subsection{Introdução}

As discretizações usualmente utilizadas em problemas de engenharia não possuem continuidade na derivada da variável principal ao longo de elementos distintos. Esse comportamento é esperado visto que suas funções de forma tem seu comportamento definido por trechos e isso introduz mais uma fonte de erro ao processo.

Os métodos mais populares para se preservar essa derivada é a utilização de splines em seus diversos tipos: Overhauser, Bezier, B-splines, Non Uniform Rational Basis Splines (NURBS), T-splines e PHT-splines. As NURBS para problemas de elasticidade bidimensional foram apresentados pela primeira vez por Simpson et al. (2012), Simpson et al. (2013).

As funções de forma lagrangianas usuais não são capazes de descrever geometrias mais complexas exatamente. Enquanto as NURBS conseguem representar, exatamente, seções cônicas, incluindo, círculos, hipérboles, elipses e parábolas. Usar a representação analítica no MEC acarreta, para geometrias complexas, em uma precisão maior para um mesmo número de elementos. As NURBS são mais adequadas para representar as geometrias e podem também ser adequadamente usadas para aproximar as variáveis de campo.

No MEC, a grande vantagem de usar as mesmas funções que descrevem a geometria para aproximar as variáveis de campo é não ter que criar uma malha específica: a mesma discretização usada no modelo geométrico, gerada nos programas de modelagem, também será usada pelo MEC.

\subsection{Curvas de Bézier}

Pierre Bézier desenvolveu curvas e superfícies de forma livre, que ficaram conhecidas pelo seu nome, para o design de automóveis enquanto trabalhava na Renault. Usando a base de Bernstein se pode definir a curva de Bézier como:

$$
P(t)=\sum_{i=0}^{n} B_{i} J_{n, i}(t), \quad 0 \leq t \leq 1
$$

onde os coeficientes $B_{i}$ são chamados de pontos de controle, que formam o polígono de controle, e a base de Bernstein $J_{n, i}(t)$ é dada por:

$$
J_{n, i}(t)=\left(\begin{array}{c}
n \\
i
\end{array}\right) t(1-t)^{n-i}
$$




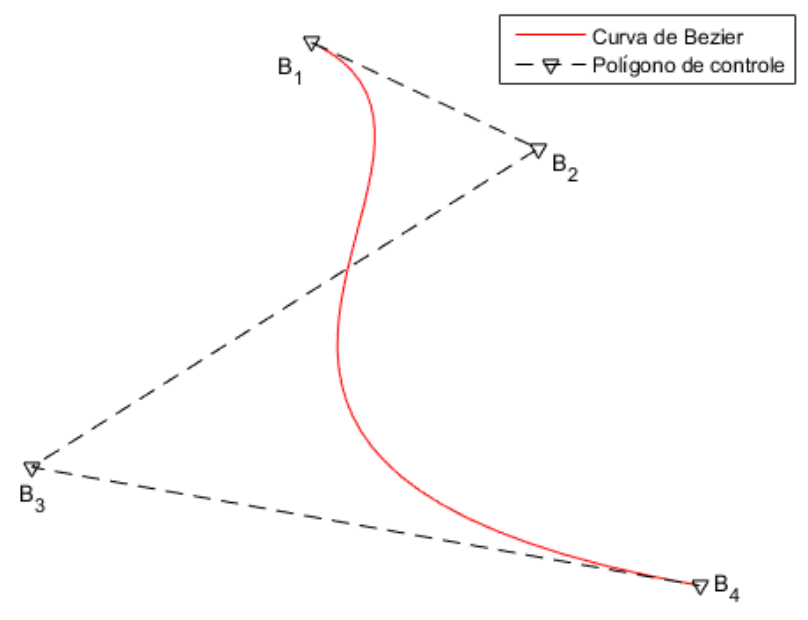

Figura 4.1: Curva de Bézier

Uma curva de Bézier de ordem $n=3$ e seu polígono de controle podem ser observados na figura 4.2. As propriedades mais importantes dessa curva são:

- O número de pontos de controle menos um é o grau do polinômio definido pela curva.

- A curva começa no primeiro ponto de controle e termina no último.

- Os vetores tangentes, no final e no início da curva, coincidem com os segmentos do polígono de controle.

- A curva está sempre contida na envoltória complexa dos pontos de controle.

- A curva não é afetada por uma transformação afim.

- Cada ponto da curva é calculado como uma soma ponderada de todos os pontos de controle. Logo, uma mudança de um ponto de controle afeta a curva globalmente.

\subsection{B-splines}

As B-splines são uma forma generalizada das curvas de Bézier. Uma B-spline é composta de uma ou mais curvas de Bézier com um mecanismo de continuidade entre os segmentos. Cada ponto de controle define e influencia apenas um segmento da curva B-spline. A curva é descrita matematicamente por:

$$
P(t)=\sum_{i=1}^{n+1} B_{i} N_{i, k}(t), \quad t_{\min } \leq t \leq t_{\max }, \quad 2 \leq k \leq n+1
$$


onde $B_{i}$ são os pontos de controle e $N_{i, k}$ são as funções de base de ordem $k$ e grau $k-1$. Essas funções não são definidas explicitamente e são calculadas recursivamente por:

$$
\begin{gathered}
N_{i, 1}(t)=\left\{\begin{array}{c}
1 \text { se } u_{i} \leq t<u_{i+1} \\
0 \text { restante do intervalo }
\end{array}\right. \\
N_{i, k}(t)=\frac{\left(t-u_{i}\right) N_{i, k-1}(t)}{u_{i+k-1}-u_{i}}+\frac{\left(u_{i+k}-t\right) N_{i+1, k-1}(t)}{u_{i+k}-u_{i+1}}
\end{gathered}
$$

onde $u_{i}$ são os valores do vetor de nós $U$. O vetor $U$ é uma série de parâmetros reais crescentes e tem uma forte influência nas funções de base de uma B-spline. O número de intervalos no vetor $U$ define o número de segmentos na curva. Cada segmento sofre influência de $k$ pontos de controle. Cada vez que o parâmetro $t$ entra em um novo segmento, um novo ponto de controle se torna ativo, enquanto um ponto antigo é descartado. O número de nós, $m$, o número de pontos de controle, $k$, e a ordem da curva, $n$, estão relacionados por:

$$
m=k+n+1
$$

Nós consecutivos podem ter o mesmo valor. Quando isso ocorre, o segmento relativo àqueles nós tem comprimento igual a zero. Consequentemente, dois pontos de controle são ativados ao mesmo tempo, e dois são desativados. Isso altera a continuidade de uma curva anteriormente suave. A quantidade de nós coincidentes é conhecida como um nó com certa multiplicidade. A multiplicidade de um nó é limitada à ordem da curva, uma vez que uma multiplicidade maior acarretaria em uma curva descontínua e pontos de controle inutilizados. Nas figuras 4.2, 4.3, 4.4 e 4.5 pode-se observar a B-spline quadrática, seus segmentos e as funções de base para dois valores distintos de $\mathbf{U}: \mathbf{U}_{1}=\left[\begin{array}{lllllllll}1 & 2 & 3 & 4 & 5 & 6 & 7 & 8 & 9\end{array}\right]$ e $\mathbf{U}_{2}=\left[\begin{array}{lllllllll}1 & 1 & 1 & 4 & 5 & 6 & 7 & 8 & 9\end{array}\right]$. 


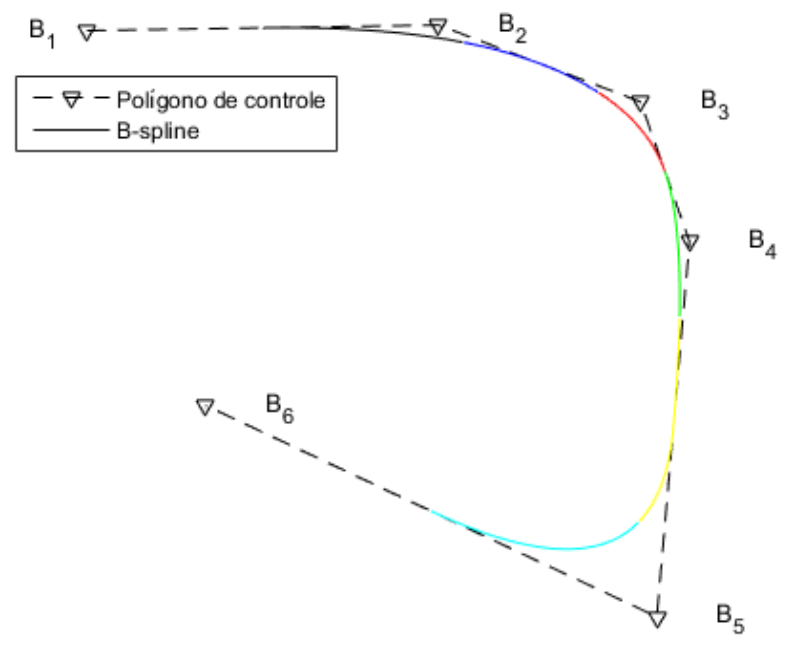

Figura 4.2: B-spline e seus segmentos para para $U_{1}$

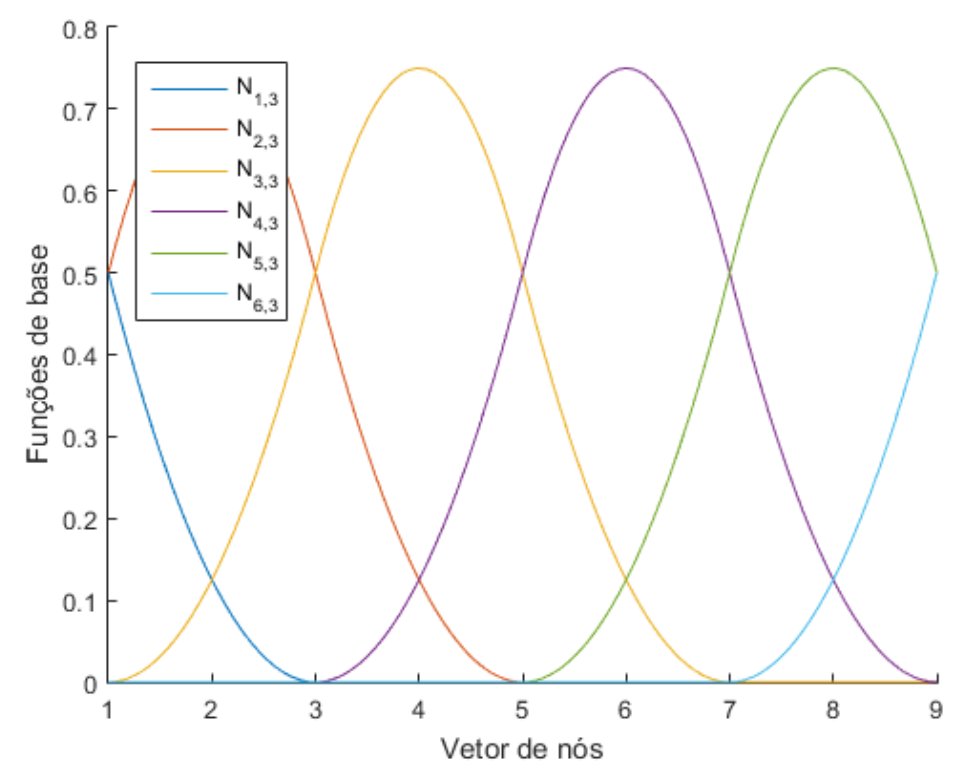

Figura 4.3: Funções de base da B-spline para $U_{1}$ 


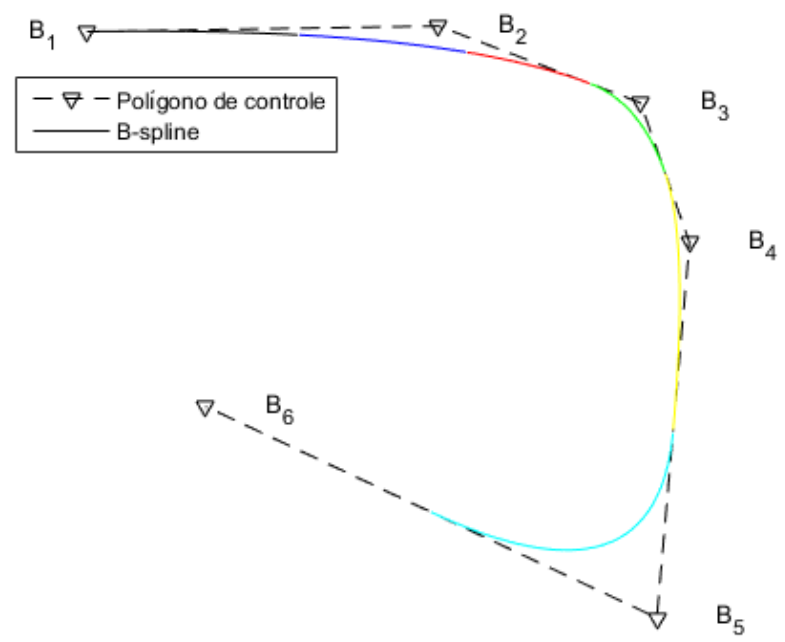

Figura 4.4: B-spline e seus segmentos para para $U_{2}$

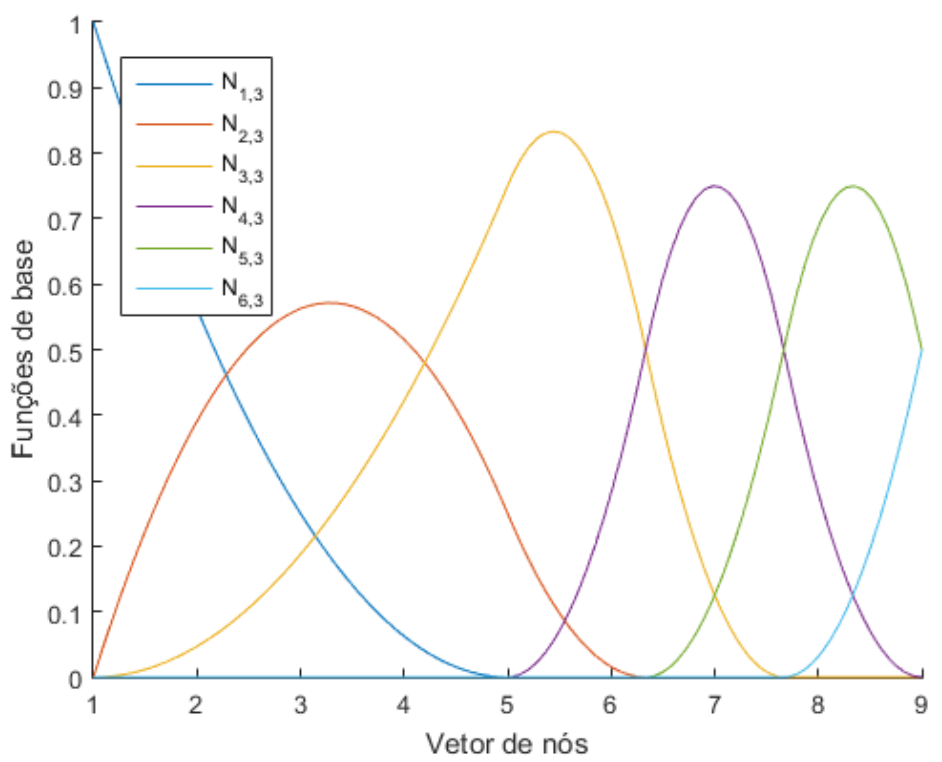

Figura 4.5: Funções de base da B-spline para $U_{2}$ 
Pode-se observar claramente o efeito do vetor U. Ao inserir uma multiplicidade de ordem 3 no início da curva, ocorre uma mudança nas funções de base e o primeiro ponto de controle passa a fazer parte da curva. Cada segmento dessa curva poderia ser representado por uma curva de Bézier, porém seriam necessários três vezes mais pontos de controle. As propriedades mais importantes dessa curva são:

- A soma das bases para qualquer valor de $t$ é igual a 1.

- As bases são sempre maiores ou iguais a zero.

- O grau da curva é um a menos que a ordem da curva. A ordem da curva não pode ser maior que o número de pontos de controle.

- A curva está sempre contida na envoltória convexa dos pontos de controle.

- A curva não é afetada por uma transformação afim.

- Cada ponto da curva é calculado como uma soma ponderada de parte dos pontos de controle. Logo, uma mudança de um ponto de controle afetará a curva apenas localmente.

\subsection{B-splines racionais não uniformes (NURBS)}

Em uma NURBS, um peso é associado à cada ponto de controle. Caso todos os pesos tenham o mesmo valor, a curva é, na realidade, uma B-spline. A maior vantagem de se trabalhar com as NURBS é representar exatamente círculos, seções cônicas e objetos de forma livre. Esse foi o maior motivo dessa representação ter sido amplamente adotada na indústria.

A maneira mais conveniente de se representar uma NURBS é usando coordenadas homogêneas. Esse sistema de coordenadas adiciona mais uma dimensão aos pontos de controle, essa relativa aos pesos:

$$
B^{h}=\left[\begin{array}{lll}
B_{x} w & B_{y} w & w
\end{array}\right],
$$

onde $B^{h}$ é o ponto de controle no sistema de coordenadas homogêneo. A equação 4.3 é, então, aplicada da mesma maneira que nas B-splines, resultando em uma B-spline que se encontra no sistema de coordenadas homogêneas. Para se obter a NURBS, se realiza a projeção dessa curva no plano $w=1$. Um ponto na projeção é obtido a partir do ponto equivalente na curva homogênea:

$$
P(t)=\left[\begin{array}{ll}
P(t)_{x}^{h} & P(t)_{y}^{h} \\
P(t)_{w}^{h} & P(t)_{w}^{h}
\end{array}\right] .
$$


Esse processo pode ser observado na figura 4.6 .

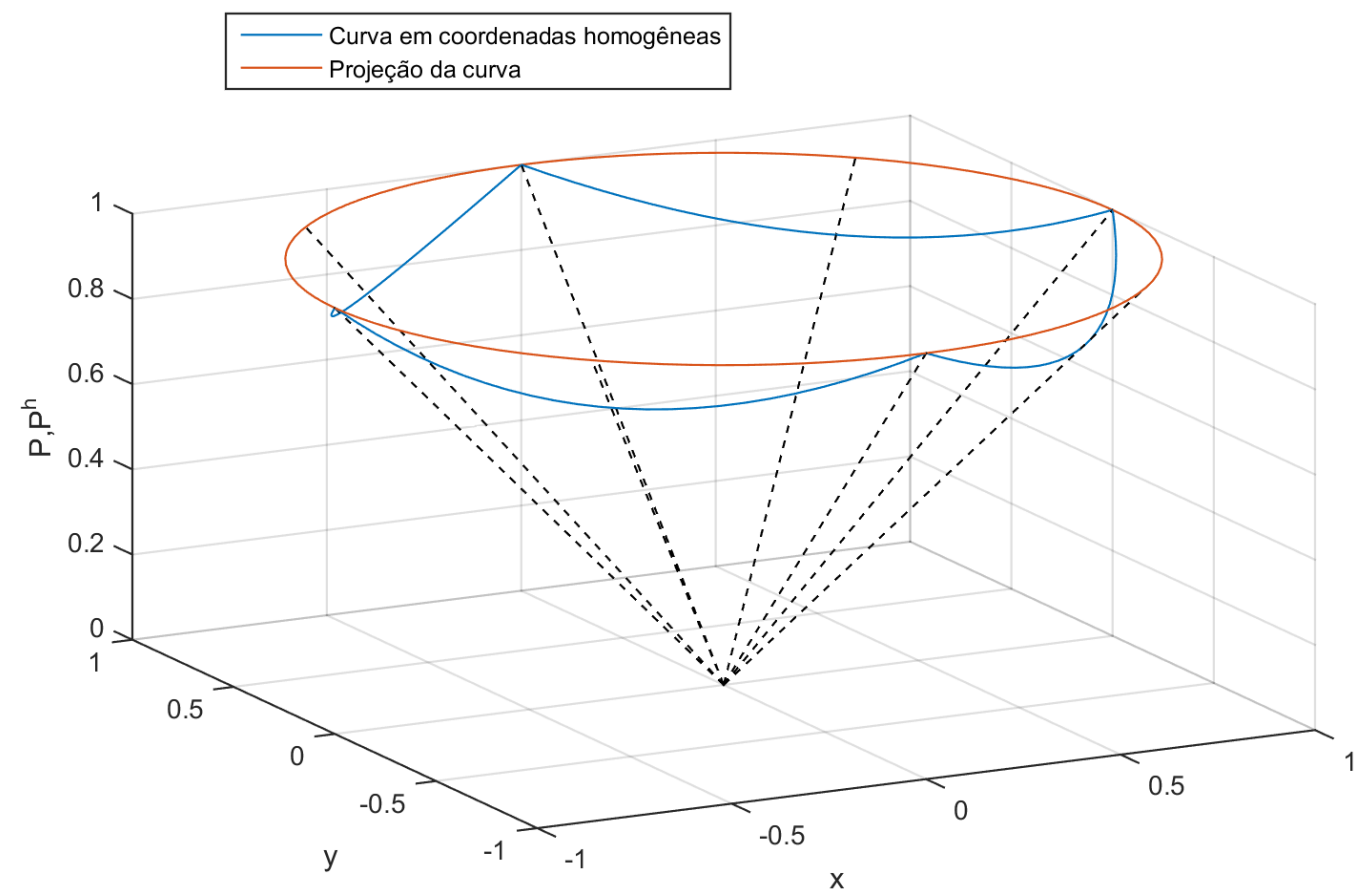

Figura 4.6: NURBS em coordenadas homogêneas.

As NURBS podem também ser calculadas pela equação:

$$
P(t)=\frac{\sum_{i=1}^{n+1} B_{i} N_{i, k}(t) w_{i}}{\sum_{i=1}^{n+1} N_{i, k}(t) w_{i}}=\sum_{i=1}^{n+1} B_{i} R_{i, k}(t),
$$

onde $R_{i, k}(t)$ é a função de base racional.

Apesar de também existir uma equação recursiva para as NURBS, essa não é maneira usual pela qual os programas comerciais calculam a curva. Estas usam, normalmente, as coordenadas homogêneas.

As NURBS são uma generalização da B-spline substituindo a base polinomial por uma razão de polinômios. Por essa razão, suas características são basicamente as mesmas.

\subsubsection{Derivadas NURBS}

As derivadas das funções de base são grandezas importantes no MEC. Como na análise isogeométrica as funções de base são as mesmas das NURBS, é essencial poder 
calculá-las.

As derivadas das funções de base das B-splines, apresentada na equação 4.3, podem ser computadas da seguinte maneira:

$$
\frac{\partial N_{i, k}(t)}{\partial t}=\frac{k}{t_{i+k}-t_{i}} N_{i, k-1}(t)-\frac{k}{t_{i+k+1}-t_{i+1}} N_{i+1, k-1}(t) .
$$

Se essa equação for substituída na equação da curva (4.3), ela resultará em:

$$
\frac{\partial P(t)}{\partial t}=\sum_{i=0}^{n-1} N_{i+1, k-1}(t) Q_{i}
$$

onde $Q_{i}$ é definido por:

$$
Q_{i}=\sum_{i=0}^{n-1} \frac{k}{t_{i+k+1}-t_{i+1}}\left(B_{i+1}-B_{i}\right) .
$$

Portanto, fica claro que a derivada de uma B-spline, de ordem $k$, também é uma B-spline, porém de ordem $k-1$, e com novos pontos de controle $Q_{i}$. Essa é uma propriedade muito interessante que possibilita que o mesmo tratamento realizado nas curvas seja utilizado nas suas derivadas.

Como a primeira derivada de uma B-spline é outra B-spline, pode-se aplicar esse técnica recursivamente para obter as derivadas de maior ordem sem maiores dificuldades.

Se o sistema de coordenadas homogêneas for utilizado, as derivadas de uma NURBS podem ser facilmente obtidas pela projeção da B-spline com maior dimensão. Considerando:

$$
P(t)=\frac{w(t) P(t)}{w(t)}=\frac{P^{h c}(t)}{w(t)}
$$

onde $P^{h c}(t)$ são as primeiras coordenadas, ignorando o peso, e $w(t)$ é a última coordenada do sistema homogêneo. Então:

$$
\frac{\partial P(t)}{\partial t}=\frac{w(t) \frac{\partial P^{h c}(t)}{\partial t}-\frac{\partial w(t)}{\partial t} P^{h c}(t)}{w(t)^{2}} .
$$

Como $P^{h c}(t)$ e $w(t)$ são as coordenadas de $P^{h}(t)$, as suas derivadas são obtidas diretamente da equação 4.11).

\subsubsection{Inserção de nós}

Nós podem ser inseridos em um vetor de nós sem mudar a geometria ou as propriedades geométricas da curva. Para cada nó inserido, um novo ponto de controle deve ser 
adicionado. Ao adicionar um novo nó $\bar{t}$ ao vetor de nós, o seguinte ponto de controle é adicionado:

$$
\bar{B}_{i}=\left\{\begin{array}{r}
B_{1} \text { se } i=1 \\
\alpha \dot{B}_{i}+(1-\alpha) B_{i-1} \text { se } 1<i<m \\
B_{n} \text { se } i=m
\end{array}\right.
$$

onde

$$
\alpha=\left\{\begin{array}{r}
1 \text { se } i=1 \\
\frac{\bar{t}-t_{i}}{t_{i+p}-\epsilon_{i}} \text { se } 1<i<m \\
0 \text { se } i=m
\end{array}\right.
$$

Os valores nodais podem ser inseridos múltiplas vezes. Porém, a continuidade das funções de base é reduzida por um a cada repetição de um nó. No entanto, ao definir o novo ponto de controle conforme as equações (4.15) e (4.16) a continuidade da curva é mantida.

\subsubsection{Decomposição de Bézier}

A decomposição de Bézier é obtida inserindo nós repetidos em todos os nós do vetor de nós até que eles tenham multiplicidade igual ao grau da curva. Na figura 4.7 pode-se observar o comportamento da curva à medida que os nós são inseridos de acordo com o algorítimo apresentado nas equações 4.15) e (4.16).

A cada nó que é inserido, a continuidade das funções de base é reduzida enquanto a curva não apresenta nenhuma alteração. A base resultante é decomposta em um conjunto de elementos de Bézier onde cada elemento corresponde a uma variação do vetor de nós.

Considerando uma curva com $n$ pontos de controle e chamando os $m$ nós necessários para realizar uma decomposição de $\bar{u}_{j}$, podemos definir $\alpha_{j}$ conforme a equação 4.16). Pode-se então escrever uma matriz que relaciona os novos pontos de controle aos antigos:

$$
\mathbf{C}^{j}=\left[\begin{array}{ccccccc}
\alpha_{1} & 1-\alpha_{2} & 0 & & \ldots & 0 \\
0 & \alpha_{2} & 1-\alpha_{3} & 0 & \ldots & 0 \\
0 & 0 & \alpha_{3} & 1-\alpha_{4} & 0 & \cdots & 0 \\
\vdots & & & & & & \vdots \\
0 & & \ldots & & \alpha_{(n+j+1)} & 1-\alpha_{(n+j)} .
\end{array}\right]
$$

A equação 4.15) pode ser rescrita em forma matricial de maneira a representar a sequência de variáveis de controle criadas por inserção de nós, como: 


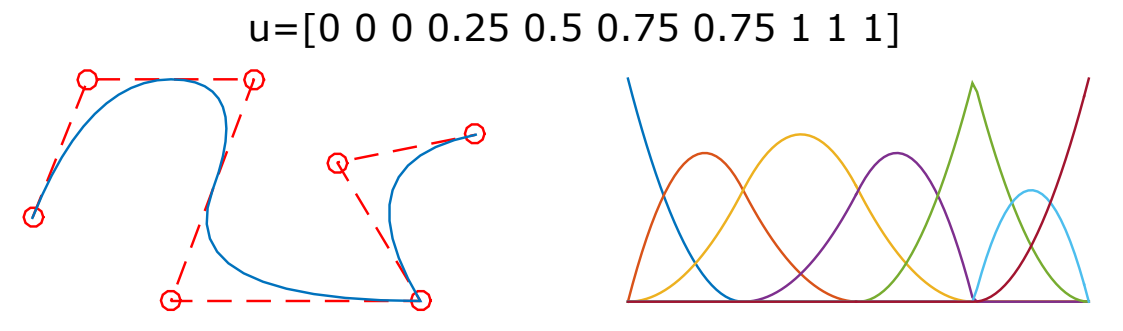

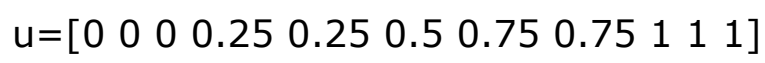
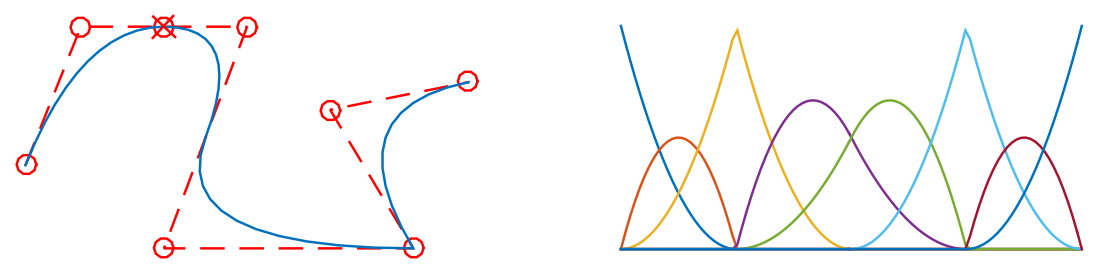

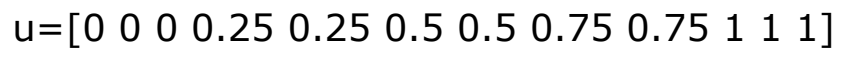
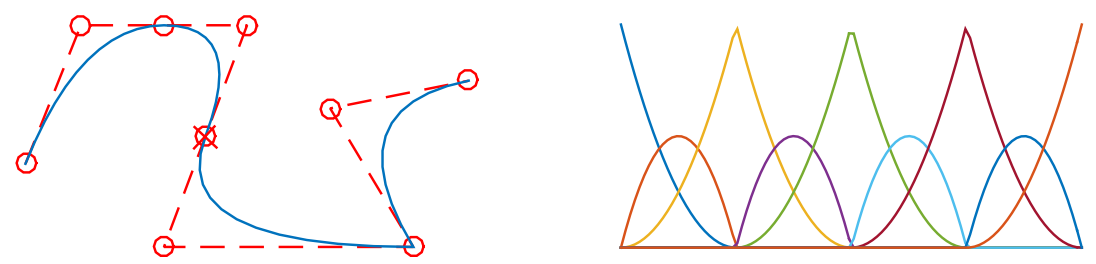

Figura 4.7: Sequência de uma curva e suas funções de base ao inserir repetidos nós

$$
\overline{\mathbf{B}}^{j+1}=\left(\mathbf{C}^{j}\right)^{t} \overline{\mathbf{B}}^{j}
$$

Ao repetir essa operação $m$ vezes, obtém-se a forma final da decomposição:

$$
\overline{\mathbf{B}}=\mathbf{C}^{t} \mathbf{B}
$$

onde $\mathbf{C}^{t}=\left(\mathbf{C}^{m}\right)^{t}\left(\mathbf{C}^{m-1}\right)^{t} \cdots\left(\mathbf{C}^{1}\right)^{t}$.

Como a inserção de nós não causa nenhuma modificação geométrica ou paramétrica, a curva de Bézier descrita pelos novos pontos de controle tem de coincidir com a spline anterior:

$$
\overline{\mathbf{B}}^{t} \mathbf{J}_{n, i}(t)=\left(\mathbf{C}^{t} \mathbf{B}\right)^{t} \mathbf{J}_{n, i}(t)=\mathbf{B}^{t} \mathbf{C} \mathbf{J}_{n, i}(t)=\mathbf{B}^{t} \mathbf{N}_{n, i}(t) .
$$

Logo, como B é arbitrário:

$$
\mathbf{C} \mathbf{J}_{n, i}(t)=\mathbf{N}_{n, i}(t)
$$

C é conhecido como operador de extração de Bézier e depende apenas do vetor de nós. 


\subsection{Superfícies NURBS}

Apenas funções splines unidirecionais e curvas foram tratadas até o momento. No entanto, superfícies e volumes também podem ser descritos usando as funções tratadas anteriormente em múltiplas direções. Uma superfície pode ser criada transladando os pontos de controle de uma curva,

$$
P(u)=\sum_{i=1}^{n+1} B_{i} R_{i, k}(u),
$$

ao longo de uma outra curva, podendo ser representado por:

$$
B_{i}(v)=\sum_{j=1}^{n+1} B_{i, j} R_{j, k}(v) .
$$

Unindo as equações anteriores, a superfície obtida por essa translação pode ser então descrita por:

$$
S(u, v)=\sum_{i=1}^{n+1} \sum_{j=1}^{n+1} R_{j, k}(v) R_{i, k}(u) B_{i, j} .
$$

Uma superfície NURBS pode ser descrita pelo produto tensorial entre duas curvas NURBS, usando dois parâmetros independentes $u$ e $v$ :

$$
S(u, v)=\sum_{i=1}^{k} \sum_{j=1}^{l} R_{i, j}(u, v) B_{i, j},
$$

onde $R_{i, j}(u, v)$ é a função de base racional para superfícies:

$$
R_{i, j}=\frac{N_{i, k_{u}}(u) N_{j, k_{v}}(v) w_{i}}{\sum_{i=1}^{k} \sum_{j=1}^{l} N_{i, k_{u}}(u) N_{j, k_{v}}(v) w_{i}} .
$$

As superfícies também podem ser calculadas usando o sistema de coordenadas homogêneas. Por utilizar o produto tensorial, essas funções de base multidirecionais herdam as propriedades do caso unidirecional. A independência linear, a propriedade de partição de unidade e o domínio de suporte compacto se mantém inalterados para o caso multidirecional (COTTRELL; HUGHES; BAZILEVS, 2009, PEIGL; TILLER, 1996, ROGERS, 2000).

As superfícies criadas com o produto tensorial não tem a capacidade de realizar um refinamento realmente local. Quando uma B-spline unidirecional é refinada, colunas ou linhas inteiras de nós são adicionadas ao espaço bidimensional. Na figura 4.8 pode-se 
observar esse comportamento quando se refina sucessivamente o canto inferior direito. Apesar de indesejado, as porções superiores direitas e inferiores esquerdas também são refinadas. Em uma superfície NURBS, todos os pontos de controle tem de estar em uma malha retangular. Essa restrição topológica faz com que um número grande de pontos de controle tenha de existir apenas para satisfaze-la. Para conseguir um refinamento realmente, local é necessário criar uma nova estrutura que não seja baseada em produtos tensoriais globais.

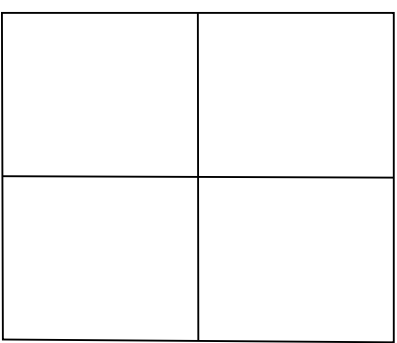

(a) Malha inicial.

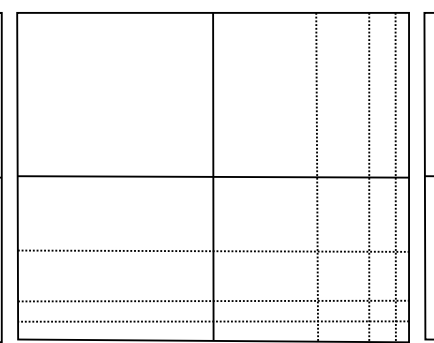

(b) Refinamento do produto tensorial.

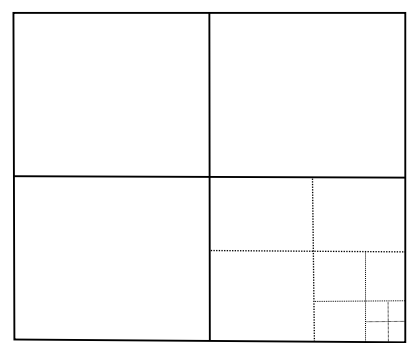

(c) Refinamento realmente local.

Figura 4.8: Falta de refinamento local nos produtos tensoriais.

As T-splines foram apresentadas inicialmente em Sederberg et al. (2003) no contexto CAD. A T-splines são uma generalização das superfícies NURBS e são capazes de diminuir consideravelmente a quantidade de pontos de controle em superfícies complexas, justamente pela propriedade de refinamento local (SEDERBERG et al., 2004). As T-splines já estão presentes em importantes softwares comerciais de CAD (AUTODESK, 2012).

Recentemente as T-splines tem sido aplicadas na análise isogeométrica nas mais diversas áreas (BAZILEVS et al., 2010, DÖRFEL; JÜTTLER; SIMEON, 2010; SCOTT et al., 2013; SCOTT et al., 2012; SCOTT et al., 2011; VERHOOSEL et al., 2011b; VERHOOSEL et al., 2011a; SIMPSON et al., 2014; DIMITRI et al., 2014; HOSSEINI et al. 2014; BAZILEVS; HSU; SCOTT, 2012; BUFFA; SANGALLI; VÁZQUEZ, 2014 , GINNIS et al., 2014). Um fato importante é que não existe garantia de que a base das T-splines seja linearmente independente e, por isso, é preciso definir uma T-spline adequada para análise o que vem sendo pesquisado extensivamente (LI; SCOTT, 2014. VEIGA et al., 2013, VEIGA et al., 2012).

Apesar das T-splines serem a alternativa mais conhecida, muitas outras foram propostas como base da análise isogeométrica. As B-splines hierárquicas tem se mostrado promissoras (KURU et al., 2014; SCHILLINGER et al., 2013; SCHILLINGER; RANK, 2011). As THB-splines, B-splines hierárquicas truncadas, (KISS; GIANNELLI; JÜTTLER, 2012, GIANNELLI; JÜTTLER; SPELEERS, 2014; BERDINSKY et al., 2014) são uma modificação das B-splines hierárquicas que formam uma partição de unidade 
e condicionamento numérico melhorado. As florestas B-splines (SCOTT; THOMAS; EVANS, 2014) são uma generalização de B-splines hierárquicas para superfícies e volumes de gênero topológico arbitrário. As PHT-spline, splines polinomiais sobre malhas T hierárquicas, (DENG et al., 2008; LI; DENG; CHEN, 2007; LI; DENG; CHEN, 2010), T-splines modificadas (KANG; CHEN; DENG, 2013) e a splines localmente refinadas (LR-splines) (DOKKEN; LYCHE; PETTERSEN, 2013, BRESSAN, 2013, JOHANNESSEN; ANDRÉ; DOKKEN, 2014) são intimamente relacionadas com as Tsplines mas com distintos níveis de suavidade e abordagem quanto ao refinamento local. Splines (MANNI; PELOSI; SAMPOLI, 2011; COSTANTINI et al., 2010) e T-splines generalizadas (BRACCO et al., 2014) incluem funções trigonométricas ou hiperbólicas às funções polinomiais permitindo uma representação exata de seções cônicas sem usar funções racionais. Em Evans et al. (2015) é proposta uma T-spline hierárquica adequada para análise, adicionando as funções de base hierárquicas às T-splines.

Fica bem evidente que ainda não existe uma tecnologia que dominará o CAD em um futuro próximo. No entanto o que elas tem em comum é que, por usar as funções de base das B-splines, elas podem ser decompostas em elementos de Bézier, o que facilita a sua utilização.

\subsection{Superfícies NURBS aparadas}

A construção de geometrias complexas usando a estrutura de produto tensorial das NURBS é um processo complexo. Uma única NURBS não consegue representrar superfícies complexas sem aparar ou unir mais de uma superfície. Para analisar superfícies complexas, distintos produtos tensoriais tem de ser unidos antes do procedimento de análise. Esse processo acarreta numa continuidade de apenas $C^{0}$ na interface dos produtos. A maneira que o CAD usualmente modela esses objetos complexos é a utilização de superfícies aparadas, o que impossibilita a utilização direta da geometria no processo de análise. O modelo original tem de ser divido em vários produtos tensoriais, que significa que o modelo original aparado tem de ser descartado e um novo modelo CAD criado. Esse processo é custoso e não pode ser realizado para qualquer modelo.

As superfícies aparadas são o método mais utilizado, atualmente, pelas aplicações CAD. No processo de aparar as superfícies, curvas definidas no domínio paramétrico são utilizadas para separar a superfície em uma parte a ser mantida e uma parte a ser aparada e descartada. Como as curvas aparadoras são criadas arbitrariamente, a geometria dos elementos aparados pode ser complexa e irregular, dificultando sua implementação no contexto dos métodos numéricos.

No CAD, as superfícies aparadas são modificadas apenas visualmente, ou seja, a parametrização se mantém a mesma e a parte aparada é omitida da vizualização. 
Logo, a topologia das NURBS não é modificada mesmo com a alteração da geometria do objeto.

As maneiras de lidar com superfícies aparadas podem ser dividas em métodos que criam um critério diferente de integração (KIM; SEO; YOUN, 2010; WANG; BENSON, 2015; NAGY; BENSON, 2015) e métodos que modificam a parametrização das NURBS (SCHMIDT; WÜCHNER; BLETZINGER, 2012, BEER; MARUSSIG; ZECHNER, 2015; MARUSSIG et al., 2016). O segundo grupo apresenta melhor estabilidade porém uma menor capacidade de ser generalizado. 


\section{MÉTODO DOS ELEMENTOS DE CONTORNO ISOGEOMÉTRICO}

\subsection{Introdução}

A formulação do método dos elementos de contorno, desenvolvida no capítulo 2 usando funções de forma polinomiais, será estendida neste capítulo para o uso das NURBS como função de forma. Esta é a principal característica do método dos elementos de contorno isogeométricos. Também será mostrado como a ACA será usada para diminuir o custo computacional da formulação apresentada.

\subsection{Formulação integral de contorno}

Conforme mostrado no capítulo 2, a equação integral de contorno do MEC, para problemas potenciais, pode ser escrita como:

$$
u(P)=\int_{s} \frac{\partial u}{\partial n} u^{*} d s-\int_{s} u \frac{\partial u^{*}}{\partial n} d s .
$$

No método isogeométrico, as variáveis são aproximadas usando as mesmas funções da geometria, no caso, as NURBS. Utilizando a equação 4.9 podemos reescrevê-la como:

$$
c u(x)=\int_{\Gamma} \sum_{i=1}^{n+1}\left(u_{i}^{c} R_{i, k}(t)\right) \frac{\partial u^{*}}{\partial n}\left(x, x^{\prime}\right) d s-\int_{\Gamma} u^{*}\left(x, x^{\prime}\right) \sum_{i=1}^{n+1}\left(\frac{\partial u_{i}^{c}}{\partial n} R_{i, k}(t)\right) d s
$$

onde $u_{i}^{c}$ e $\frac{\partial u_{i}^{c}}{\partial n}$ são a temperatura e o fluxo correspondente no ponto de controle $i$. Como $u_{i}^{c}$ e $\frac{\partial u_{i}^{c}}{\partial n}$ são valores nodais, podemos reorganizar da seguinte maneira:

$$
c u(x)=\sum_{i=1}^{n+1}\left(u_{i}^{c} \int_{\Gamma} R_{i, k}(t) \frac{\partial u^{*}}{\partial n}\left(x, x^{\prime}\right) d \Gamma\right)-\sum_{i=1}^{n+1}\left(\frac{\partial u_{i}^{c}}{\partial n} \int_{\Gamma} u^{*}\left(x, x^{\prime}\right) R_{i, k}(t) d \Gamma\right) .
$$

Na equação (5.3) não há nenhuma aproximação na geometria, somente nas variáveis $u$ e $\frac{\partial u}{\partial n}$. O contorno é parametrizado por $t$ por meio da equação 4.9 e portanto:

$$
c u(x)=\sum_{i=1}^{n+1}\left(u_{i}^{c} \int_{t_{\min }}^{t_{\max }} R_{i, k}(t) \frac{\partial u^{*}}{\partial n}\left(x, x^{\prime}\right) \frac{d \Gamma}{d t} d t\right)-\sum_{i=1}^{n+1}\left(\frac{\partial u_{i}^{c}}{\partial n} \int_{t_{\min }}^{t_{\max }} u^{*}\left(x, x^{\prime}\right) R_{i, k}(t) \frac{d \Gamma}{d t} d t\right)
$$


onde

$$
\frac{d \Gamma}{d t}=\sqrt{\left(\frac{d x(t)}{d t}\right)^{2}+\left(\frac{d y(t)}{d t}\right)^{2}} .
$$

Cada função de base é não nula apenas em um intervalo único porém intersectante. Esse intervalo pode ser entendido como o domínio de influência daquele ponto de controle. Esse domínio começa em $t_{i}$ e termina em $t_{i+k}$. Nessa formulação isogeométrica, não se define elementos, já que eles não seriam independentes: as integrais são regidas apenas pelo domínio de influência. Elas podem ser reduzidas para somente os intervalos não-nulos:

$c u(x)=\sum_{i=1}^{n+1}\left(u_{i}^{c} \int_{t_{i}}^{t_{i+k}} R_{i, k}(t) \frac{\partial u^{*}}{\partial n}\left(x, x^{\prime}\right) \frac{d \Gamma}{d t} d t\right)-\sum_{i=1}^{n+1}\left(\frac{\partial u_{i}^{c}}{\partial n} \int_{t_{i}}^{t_{i+k}} u^{*}\left(x, x^{\prime}\right) R_{i, k}(t) \frac{d \Gamma}{d t} d t\right)$.

Ao trabalhar dessa maneira torna-se simples calcular somente uma vez as funções de base e propriedades geométricas dos pontos de Gauss para cada coluna. É importante ressaltar que é mais caro calcular as funções de base das NURBS do que as funções polinomiais regulares, tornando-se interessante tomar essas precauções.

Para se calcular as integrais numericamente, mais uma mudança de variáveis é necessária. Regularizando o intervalo de integração possibilitando usar a quadraturas de Gauss.

$c u(x)=\sum_{i=1}^{n+1}\left(u_{i}^{c} \int_{-1}^{1} R_{i, k}(t) \frac{\partial u^{*}}{\partial n}\left(x, x^{\prime}\right) \frac{d \Gamma}{d t} \frac{d t}{d \xi} d \xi\right)-\sum_{i=1}^{n+1}\left(\frac{\partial u_{i}^{c}}{\partial n} \int_{-1}^{1} u^{*}\left(x, x^{\prime}\right) R_{i, k}(t) \frac{d \Gamma}{d t} \frac{d t}{d \xi} d \xi\right)$

onde

$$
\frac{d t}{d \xi}=\frac{t_{i+k}-t_{i}}{2}
$$

A equação pode ser então reorganizada com os termos da matriz $\mathbf{H}$ de um lado e os termos da matriz $\mathbf{G}$ do outro:

$$
\begin{aligned}
\sum_{i=1}^{n+1}\left(u_{i}^{c}\left(\int_{-1}^{1} R_{i, k}(t) \frac{\partial u^{*}}{\partial n}\left(x, x^{\prime}\right) \frac{d \Gamma}{d t} \frac{d t}{d \xi} d \xi-c R_{i, k}\left(x^{\prime}\right)\right)\right)= & \\
& \sum_{i=1}^{n+1}\left(\frac{\partial u_{i}^{c}}{\partial n} \int_{-1}^{1} u^{*}\left(x, x^{\prime}\right) R_{i, k}(t) \frac{d \Gamma}{d t} \frac{d t}{d \xi} d \xi\right)
\end{aligned}
$$

Nota-se que o termo $c$, terá influência em $k$ elementos da matriz $\mathbf{H}$, o que não ocorre no método tradicional.

Esse procedimento pode ser realizado sem grandes modificações para o caso tridimensional atentando, apenas, que as integrais de linha são substituídas por integrais 
de superfície. O que resulta em:

$$
\begin{aligned}
\sum_{i=1}^{n+1}\left(u_{i}^{c}\left(\int_{-1}^{1} \int_{-1}^{1} R_{i, k}(t) \frac{\partial u^{*}}{\partial n}\left(x, x^{\prime}\right) J \frac{d u}{d \xi} \frac{d v}{d \eta} d \xi d \eta-c R_{i, k}\left(x^{\prime}\right)\right)\right)= \\
\sum_{i=1}^{n+1}\left(\frac{\partial u_{i}^{c}}{\partial n} \int_{-1}^{1} \int_{-1}^{1} u^{*}\left(x, x^{\prime}\right) R_{i, k}(t) J \frac{d u}{d \xi} \frac{d v}{d \eta} d \xi d \eta\right) .
\end{aligned}
$$

As equações elásticas podem ser obtidas pelo mesmo processo que foi apresentado para o caso potencial.

\subsection{Integrais fracamente singulares}

Devido a característica singular das soluções fundamentais, quando o ponto fonte pertencer ao domínio de influência, o integrando será singular e deverá ter um tratamento especial. Uma técnica bastante utilizada no MEC para calcular indiretamente as integrais singulares são a consideração de um corpo de temperatura constante, no caso de problemas potenciais, e o deslocamento de corpo rígido, no caso de problemas elásticos. Essa técnica consiste em calcular primeiro todas as integrais não-singulares para um ponto de colocação em específico, e então o deslocamento de corpo rígido é aplicado para calcular a integral restante. Em funções polinomiais o número de integrais singulares é igual ao número de translações de corpo rígido. Isso ocorre porque, no MEC convencional, para cada ponto de colocação e o elemento singular correspondente, somente uma função de base associada aquele elemento é não nula. Portanto só existe uma integral singular em cada elemento.

Nas NURBS, as funções de base não tem essa característica, várias delas elas são não nulas nos pontos de colocação. Para cada ponto de colocação se tem $k$ integrais singulares que precisam de tratamento especial.

As integrais de natureza fracamente singular são calculadas pela transformada proposta por Telles (1987). O método aplica uma transformação cúbica de coordenadas de forma que as abscissas se concentram em torno da singularidade, e faz com que o jacobiano dessa transformação se anule no ponto singular. Isso é obtido por meio da seguinte transformação:

$$
\hat{\xi}=\frac{\left(\gamma-\gamma^{\prime}\right)^{3}+\gamma^{\prime}\left(\gamma^{\prime 2}+3\right)}{1+3 \gamma^{\prime 2}}
$$

onde

$$
\xi^{\prime}=\sqrt[3]{\hat{\xi}^{\prime}\left(\hat{\xi}^{\prime 2}-1\right)+\left|\hat{\xi}^{\prime 2}-1\right|}+\sqrt[3]{\hat{\xi}^{\prime}\left(\hat{\xi}^{\prime 2}-1\right)+\left|\hat{\xi}^{\prime 2}-1\right|+\hat{\xi}^{\prime}}
$$

$\hat{\xi}^{\prime}$ é a localização da singularidade no espaço original e $\gamma$ representa a nova variável de 
integração. Portanto, um jacobiano dessa transformação é dado por:

$$
d \hat{\xi}=\frac{3\left(\gamma-\gamma^{\prime}\right)^{2}}{1+3 \gamma^{\prime 2}} d \gamma
$$

Como pôde ser observado, a formulação apresentada é unidimensional, porém a extensão para duas dimensões é direta, pois para cada conjunto de abcissas é transformado separadamente e os pesos multiplicados pelo jacobiano e depois utilizados conforme ocorre na extensão da quadratura de Gauss-Legendre para duas dimensões.

\subsection{Integrais fortemente singulares}

Em Ubessi (2014), é apresentado um método com avaliação direta das integrais fortemente singulares. O método consiste em realizar expansões assintóticas dos integrandos da equação de equilíbrio do MEC e extrair a parte singular do núcleo para que a mesma seja integrada analiticamente. Isto é feito de forma generalizada e considerando o mapeamento dos elementos, não dependendo de formulações específicas para cada tipo de elemento. O método foi apresentado primeiramente por Guiggiani e Casalini (1987) e com maior detalhamento em Guiggiani (1998).

No MEC bidimensional, o método é aplicado a uma integral sobre um elemento de contorno em suas coordenadas normalizadas:

$$
\int_{\Gamma} K_{i j} d \Gamma=\int_{-1}^{1} T_{i j}(p, Q) \phi_{a}(\xi) J_{n}(\xi) d \xi
$$

onde $\phi_{a}$ é uma função de interpolação associado ao nó $a$ e $J_{n}$ é o determinante do Jacobiano do mapeamento geométrico. Portanto, o integrando da equação (5.14) já leva em consideração a representação da geometria do problema. O primeiro passo do método direto é expandir assintoticamente o núcleo em série de Laurent em torno do ponto-fonte (GUIGGIANI, 1998):

$$
K_{i j}(\xi, \eta)=\frac{\mathbf{F}_{-1}(\eta)}{\rho}+\frac{\mathbf{F}_{-2}(\eta)}{\rho^{2}}+\cdots+O(1)
$$

onde $\rho=\xi-\eta$ é a imagem do raio e $\eta$ é a posição do ponto fonte no domínio regularizado. Esta expansão separa as parcelas fortemente singular

A equação (5.14) pode ser subtraída e somada pelos primeiros termos da expansão (5.15) sem alteração do seu conteúdo. A subtração promove a regularização do núcleo singular da integral equanto os termos adicionais tem tratamento analítico.

A expressão final dada por Guiggiani (1998) para um elemento que contém o ponto 
singular é:

$$
\begin{array}{r}
\int_{\Gamma} K_{i j} d \Gamma=\int_{\Gamma}\left[K_{i j}-\left(\frac{\mathbf{F}_{-1}(\eta)}{\rho}+\frac{\mathbf{F}_{-2}(\eta)}{\rho^{2}}\right)\right] d \Gamma+\mathbf{F}_{-1} \log \left|\frac{1-\eta}{-1-\eta}\right|+ \\
\mathbf{F}_{-2}\left(-\frac{1}{1-\eta}+\frac{1}{-1-\eta}\right)
\end{array}
$$

A dedução da equação (5.16) não possui nenhuma aproximação em relação a sua forma original. Como o termo integral possui um termo que promove o cancelamento da singularidade, ele é regular e pode ser calculado pela quadratura gaussiana padrão. A aplicação genérica do método depende apenas do conhecimento das expansões $\mathbf{F}_{-1}$ e $\mathbf{F}_{-2}$ do integrando da equação (5.14).

Em Marczak e Creus (2002) encontram-se os valores $\mathbf{F}_{-1}=0$ e $\mathbf{F}_{-2}=0$ para a solução fundamental de fluxo para problemas potenciais, enquanto para a solução fundamental de forças de superfície para problemas elásticos bidimensionais, tem-se:

$$
\begin{gathered}
\mathbf{F}_{-1}=-\frac{1-2 \nu}{4 \pi(1-\nu)}\left[n_{\alpha}(p) t_{\beta}(p)-n_{\beta}(p) t_{\alpha}(p)\right] \phi_{a}(\eta) \\
\mathbf{F}_{-2}=0 .
\end{gathered}
$$

Fica claro, ao analisar os valores de $\mathbf{F}_{-1}$ e $\mathbf{F}_{-2}$, que a solução fundamental de fluxo é regular. Por outro lado, a solução de forças de superfície é fortemente singular.

Para o caso tridimensional, um processo semelhante é realizado, o elemento é mapeado em elemento retangular padrão:

$$
\int_{\Gamma} K_{i j} d \Gamma=\int_{-1}^{1} \int_{-1}^{1} T_{i j}(p, Q) \phi_{a}(\xi) J_{n}(\xi) d \xi_{1} d \xi_{2}
$$

e, depois, em coordenadas polares $(\rho, \theta)$ centradas em $\eta$ tal que $d \xi_{1} d \xi_{2}=\rho d \rho d \theta$. A equação 5.19 torna-se, então:

$$
\int_{0}^{2 \pi} \int_{0}^{\rho} T_{i j}(p, Q) \phi_{a}(\xi) J_{n}(\xi) \rho d \rho d \theta
$$

Subtraindo e somando os primeiros termos da expansão na equação 5.20 obtém-se a forma regularizada da equação:

$$
\int_{0}^{2 \pi} \int_{0}^{\rho} K_{i j} d \rho d \theta=\int_{0}^{2 \pi} \int_{0}^{\rho}\left[K_{i j}-\left(\frac{\mathbf{F}_{-1}(\eta)}{\rho}+\frac{\mathbf{F}_{-2}(\eta)}{\rho^{2}}\right)+\left(\frac{\mathbf{F}_{-1}(\eta)}{\rho}+\frac{\mathbf{F}_{-2}(\eta)}{\rho^{2}}\right)\right] d \rho d \theta
$$


A expressão final dada por Ubessi (2014) é dada por:

$$
\begin{aligned}
\int_{0}^{2 \pi} \int_{0}^{\rho} K_{i j} d \rho d \theta=\int_{0}^{2 \pi} \int_{0}^{\rho}\left[K_{i j}-\right. & \left.\left(\frac{\mathbf{F}_{-1}(\eta)}{\rho}+\frac{\mathbf{F}_{-2}(\eta)}{\rho^{2}}\right)\right] d \rho d \theta+ \\
& \int_{0}^{2 \pi}\left[\mathbf{F}_{-1}(\eta) \log \left|\frac{\rho}{\beta}\right|-\mathbf{F}_{-2}(\eta)\left(\frac{\gamma}{\beta^{2}}+\frac{1}{\rho}\right)\right] d \theta
\end{aligned}
$$

onde $\beta=\frac{1}{A}$ e $\gamma=-\frac{C}{A^{4}}$ são os coeficientes do primeiro e segundo termo da expansão de $\rho$.

No núcleo singular referente às forças de superfície, temos que $\mathbf{F}_{-2}=0$ e que:

$$
\mathbf{F}_{-1}=\frac{-(1-2 \nu)}{8 \pi(1-\nu)}\left(n_{\alpha} A_{\beta}-n_{\beta} A_{\alpha}\right) \frac{\phi(\eta) J(\eta)}{A^{3}}
$$

Os parâmetros $A, B$ e $C$ são definidos como:

$$
\begin{gathered}
A_{i}=x_{i, 1} \cos (\theta)+x_{i, 2} \sin (\theta), \\
B_{i}=x_{i, 11} \frac{\cos ^{2}(\theta)}{2}+x_{i, 12} \sin (\theta) \cos (\theta)+x_{i, 22} \frac{\sin ^{2}(\theta)}{2}, \\
A=\left\|A_{i}\right\|
\end{gathered}
$$

e

$$
C=A_{i} B_{i}
$$

Dada a falta de generalidade da técnica de imposição de movimento do corpo rígido e as limitações da quadratura de Kutt (1975), o método direto se apresenta como o mais adequado no tratamento das singularidades numa formulação isogeométrica e por isso foi utilizado nesse trabalho.

\subsection{Pontos de colocação}

Os pontos de controle e nós utilizados para definir as NURBS não são necessariamente únicos, como já foi mencionado; eles podem ter uma multiplicidade de até o grau da curva. Além disso os pontos de controle não estão na curva e sim no polígono de controle. Essas características fazem com que essas entidades não sejam as melhores escolhas para os pontos de colocação do MEC.

Em Li e Qian (2011) são testadas diferentes alternativas como pontos de colocação: uniforme, pontos de Gauss, valores máximos das funções de base e as coordenadas de Greville. Naquela análise, as abcissas de Greville se mostram como a melhor alternativa para resultados estáveis e precisos no método isogeométrico.

A abscissa de Greville é o ponto no domínio sobre o qual o ponto de controle tem 
influência máxima (FARIN, 1996) e é definida como a média de $d$ nós:

$$
\gamma_{i}=\frac{1}{d}\left(t_{i+1}+\ldots+t_{i+d}\right)
$$

Esses pontos são únicos, localizados no contorno e tem a mesma quantidade que os pontos de controle. Quando a curva é suave, eles são os candidatos perfeitos para serem usados como pontos de colocação, e são assim utilizados em Simpson et al. (2012) e Scott et al. (2013). Entretanto, quando cantos estão presentes, especialmente para problemas tridimensionais, se torna problemático calcular os termos diagonais uma vez que os pontos de colocação estarão nos cantos.

Para contornar esse obstáculo, propõe-se uma mudança nas posições do primeiro e do último ponto de colocação:

$$
\begin{gathered}
\gamma_{1}=\gamma_{1}+\beta\left(\gamma_{2}-\gamma_{1}\right), \\
\gamma_{n}=\gamma_{n}-\beta\left(\gamma_{n}-\gamma_{n-1}\right),
\end{gathered}
$$

onde $\beta$ é um coeficiente que define o quanto o ponto de colocação se move. Em Wang e Benson (2015) fica claro que o valor ótimo é $\beta=0.5$.

Esse procedimento lembra o uso de elementos descontínuos porém, como não há nenhuma mudança nos pontos de controle, não haverá nenhuma perda de continuidade na aproximação.

\subsection{Condição de contorno}

Como os ponto de controle estão tipicamente fora do contorno, as condições de contorno não podem ser aplicadas diretamente. Para superar esse problema um matriz de transformação $E$ para B-splines é proposta em Cabral et al. (1990). Essa matriz usa as funções de base para relacionar os valores nos pontos de controle com os valores nos pontos de colocação.

$$
\begin{array}{r}
\mathbf{u}=\mathbf{E u}_{c} \\
\frac{\partial \mathbf{u}}{\partial \mathbf{n}}=\mathbf{E} \frac{\partial \mathbf{u}_{\mathbf{c}}}{\partial \mathbf{n}}
\end{array}
$$

onde $\mathbf{u}$ é um vetor que contém os valores nos pontos de colocação e $\mathbf{u}_{c}$ é um vetor que contém os valores nos pontos de controle. A mesma ideia pode ser aplicada às NURBS.

A equação de equilíbrio obtida pelo MEC isogeométrico é escrita em termos da 


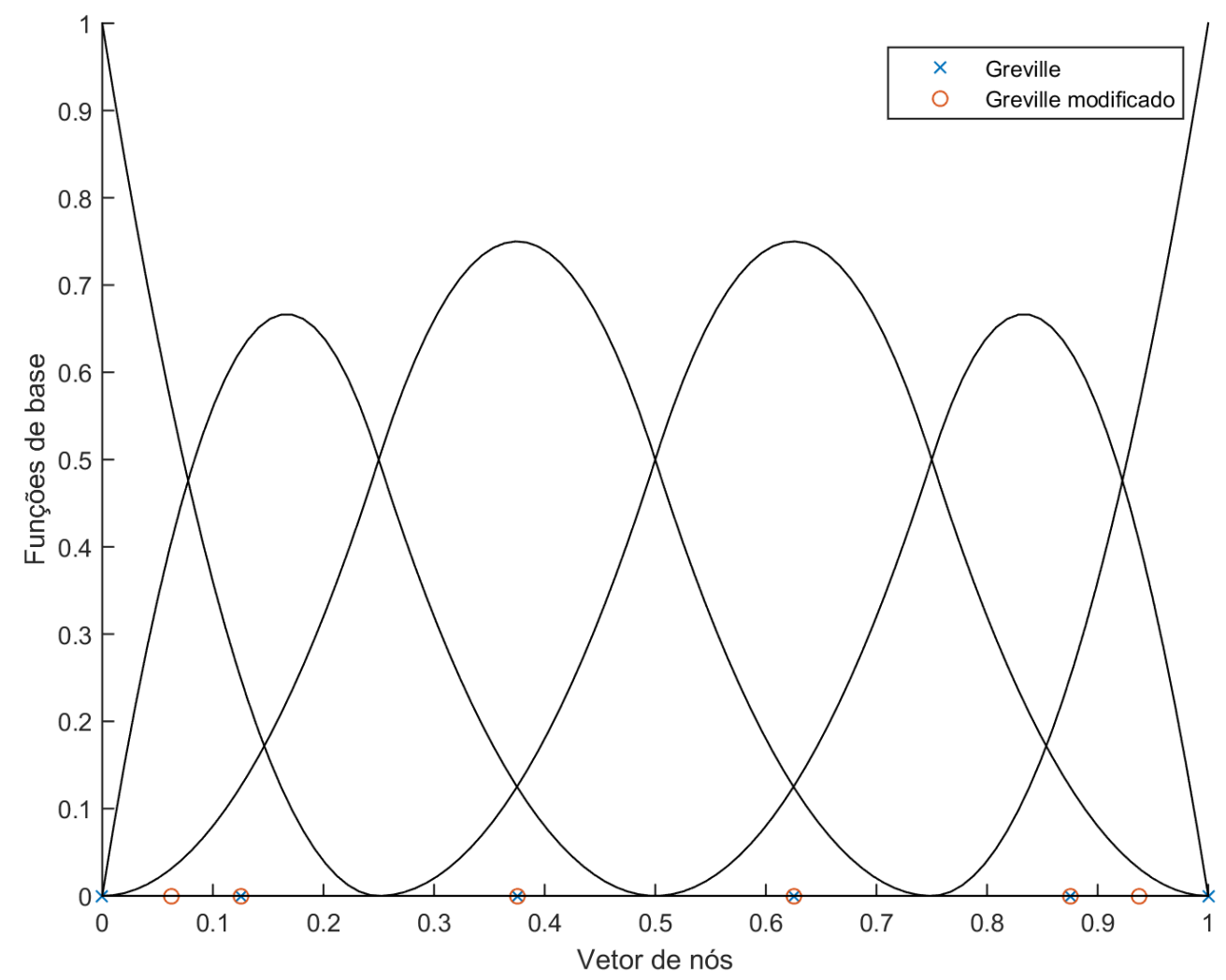

Figura 5.1: Modificação das abcissas de Greville.

pontos de controle:

$$
\mathbf{H} \mathbf{u}_{c}=\mathbf{G} \frac{\partial \mathbf{u}_{\mathbf{c}}}{\partial \mathbf{n}}
$$

Aplicando a matriz de transformação, a equação pode ser reescrita por:

$$
\mathbf{H E}^{-1} \mathbf{u}=\mathbf{G E}^{-1} \frac{\partial \mathbf{u}}{\partial \mathbf{n}}
$$

Essa equação já pode ser resolvida da maneira usual do MEC. No entanto, se o ACA estiver sendo utilizado, a inversa da matriz não é esparsa e o método seria prejudicado uma vez que as matrizes hierárquicas $\mathbf{H}$ e $\mathbf{G}$ se tornariam matrizes cheias depois de ser multiplicados por $\mathbf{E}^{-1}$. Além disso o custo do cálculo da inversa, de ordem $n^{3}$, se torna proibitivo a medida que se aumenta o tamanho do problema.

A alternativa proposta é adicionar um nó com máxima multiplicidade para cada mudança no tipo de condição de contorno. Ao fazer isso, cada intervalo com diferentes tipos de condição de contorno podem ser separados e os valores nos pontos de controle podem ser encontrados, solucionando um simples sistema linear para cada intervalo: 


$$
\begin{array}{r}
\mathbf{u}_{c}=\left[\mathbf{E}_{0}^{-1} \mathbf{u}_{0} \cdots \mathbf{E}_{i}^{-1} \mathbf{u}_{i} \cdots \mathbf{E}_{n}^{-1} \mathbf{u}_{n}\right] \\
\frac{\partial \mathbf{u}_{\mathbf{c}}}{\partial \mathbf{n}}=\left[\mathbf{E}_{0}^{-1} \frac{\partial \mathbf{u}_{\mathbf{0}}}{\partial \mathbf{n}} \cdots \mathbf{E}_{i}^{-1} \frac{\partial \mathbf{u}_{\mathbf{i}}}{\partial \mathbf{n}} \cdots \mathbf{E}_{n}^{-1} \frac{\partial \mathbf{u}_{\mathbf{n}}}{\partial \mathbf{n}}\right]
\end{array}
$$

Fazendo isso, nos segmentos conhecidos, a condição de contorno fica conhecida nos pontos de controle. Pode-se, portanto, aplicar o procedimento padrão do MEC na equação (5.32). A solução, porém, será encontrada em termos dos pontos de controle, devendo ainda ser levada ao contorno por meio da equação (5.32). Esse procedimento não afeta a geometria. Porém, nos pontos onde a mudança do tipo de condição de contorno ocorre, haverá apenas continuidade $C_{0}$, o que, na maioria dos casos, não deve ser um problema.

Ao usar matrizes hierárquicas, a troca de colunas entre as matrizes $\mathbf{H}$ e $\mathbf{G}$ não pode ser feita de maneira simples, visto que as matrizes estão armazenadas em blocos. A maneira mais simples é calcular diretamente as matrizes $\mathbf{A}$ e o vetor $\mathbf{b}$ ao invés das matrizes $\mathbf{H}$ e $\mathbf{G}$.

Em Brancati, Aliabadi e Benedetti (2009), um outro procedimento é apresentado para problemas acústicos. Ele consiste em trocar os valores correspondentes a condição de contorno na aproximação de baixo posto por zeros:

$$
\left[\begin{array}{ll}
x & x \\
x & x \\
x & x \\
x & x \\
x & x
\end{array}\right] \quad\left[\begin{array}{lllll}
x & x & x & 0 & x \\
x & x & x & 0 & x
\end{array}\right]
$$

e adicionar os valores que deveriam ser trocados:

$$
\left[\begin{array}{lll}
x & x & t \\
x & x & t \\
x & x & t \\
x & x & t \\
x & x & t
\end{array}\right] \quad\left[\begin{array}{lllll}
x & x & x & 0 & x \\
x & x & x & 0 & x \\
0 & 0 & 0 & 1 & 0
\end{array}\right] .
$$

Esse procedimento aumenta o tamanho da aproximação mas pode ser facilmente implementado, sendo vantajoso quando se tem de aplicar várias diferentes condições de contorno ao mesmo problema. 


\subsection{Tensão no contorno}

Uma maneira simples e eficiente de recuperar as tensões no contorno é usando a lei de Hooke e a formula de Cauchy a partir do deslocamento, das derivadas do deslocamento e das forças de superfície. Para problemas bidimensionais, se define um sistema de coordenadas tal que $\mathbf{e}_{1}$ é um vetor unitário na direção normal e $\mathbf{e}_{2}$ é um vetor unitário na direção tangente como apresentado na figura 5.2. Esse vetores podem ser obtidos por:

$$
\begin{gathered}
\mathbf{e}_{1}=\mathbf{n}, \\
\mathbf{e}_{2}=\frac{\mathbf{m}}{|\mathbf{m}|},
\end{gathered}
$$

onde $\mathbf{n}$ é o vetor normal e $\mathbf{m}$ o vetor tangencial,

$$
\mathbf{m}=\frac{d x}{d \xi} .
$$

A matriz de transformação do sistema global para o sistema tangencial local é

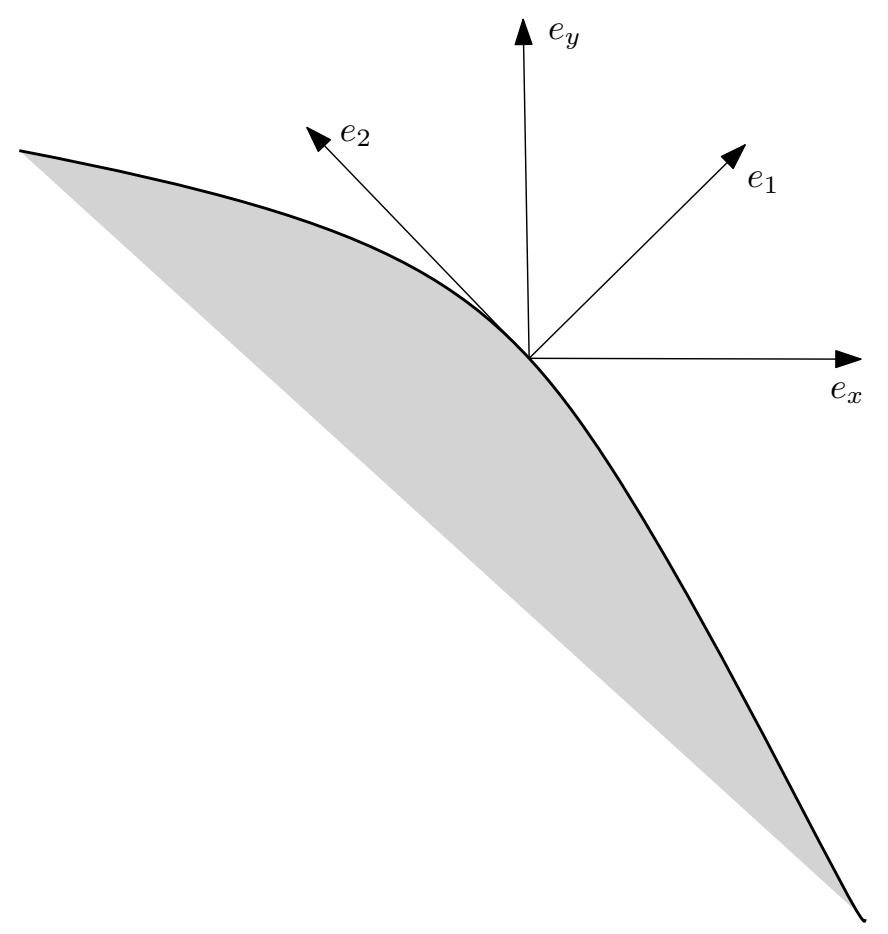

Figura 5.2: Sistema de coordenadas tangenciais bidimensionais.

$$
\mathbf{A}=\left[\begin{array}{l}
\mathbf{e}_{1} \\
\mathbf{e}_{2}
\end{array}\right]
$$


O tensor de tensões em coordenadas locais é dado por:

$$
\begin{gathered}
\sigma_{11}=t_{1}, \\
\sigma_{12}=t_{2}, \\
\sigma_{22}=\frac{E}{1-\nu^{2}} \epsilon_{22}+\frac{\nu}{1-\nu} t_{1},
\end{gathered}
$$

onde a deformação é dada por:

$$
\epsilon_{22}=A_{2 j} \frac{\partial u_{j}}{\partial \xi} \frac{\partial \xi}{\partial x_{2}}
$$

Finalmente, a tensão, em coordenadas globais, pode ser obtida como:

$$
\sigma_{i j}=\mathbf{A}_{k i} \mathbf{A}_{n j} \sigma_{k n}
$$

Para um problema tridimensional, o procedimento é semelhante. O primeiro passo é construir um sistema de coordenadas tangencial:

$$
\begin{gathered}
\mathbf{m}_{\mathbf{1}}=\frac{d x}{d \xi_{1}}, \\
\mathbf{m}_{\mathbf{2}}=\frac{d x}{d \xi_{2}} \\
\mathbf{n}=\mathbf{m}_{1} \times \mathbf{m}_{2} .
\end{gathered}
$$

onde $\mathbf{m}_{\mathbf{1}}$ e $\mathbf{m}_{\mathbf{2}}$ são os dois vetores tangencias e $\mathbf{n}$ um vetor normal a superfície. Esse sistema não é ortogonal nem unitário, para se obter um sistema com essas características, como apresentado na figura 5.3 , define-se os vetores unitários do sistema como:

$$
\begin{gathered}
\mathbf{e}_{1}=\frac{\mathbf{m}_{1}}{\left|\mathbf{m}_{1}\right|}, \\
\mathbf{e}_{3}=\frac{\mathbf{n}}{|\mathbf{n}|}, \\
\mathbf{e}_{2}=\mathbf{e}_{1} \times \mathbf{e}_{2} .
\end{gathered}
$$

E o tensor de rotação pode ser escrito da seguinte forma matricial:

$$
\mathbf{A}=\left[\begin{array}{l}
\mathbf{e}_{1} \\
\mathbf{e}_{2} \\
\mathbf{e}_{3}
\end{array}\right]
$$

Das equações constitutivas e das relações entre tensão e forças de superfície pode-se 


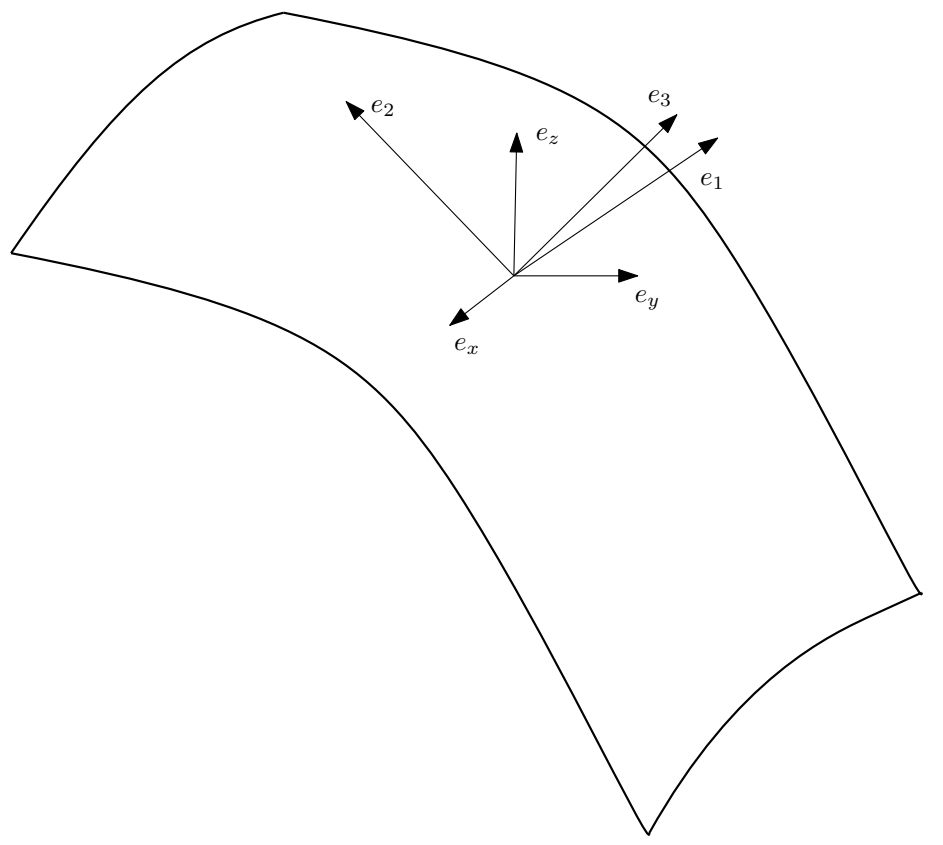

Figura 5.3: Sistema de coordenadas tangenciais tridimensionais.

obter as tensões no sistema tangencial-ortogonal como:

$$
\begin{gathered}
\sigma_{33}=t_{3}, \\
\sigma_{23}=t_{2}, \\
\sigma_{13}=t_{1}, \\
\sigma_{11}=\frac{E}{1-\nu^{2}}\left(\epsilon_{11}+\nu \epsilon_{22}\right)+\frac{\nu}{1-\nu} t_{3}, \\
\sigma_{12}=\frac{E}{1+\nu} \epsilon_{12} \\
\sigma_{22}=\frac{E}{1-\nu^{2}}\left(\epsilon_{22}+\nu \epsilon_{11}\right)+\frac{\nu}{1-\nu} t_{3},
\end{gathered}
$$

onde as deformações são dadas por:

$$
\begin{aligned}
\epsilon_{11} & =A_{1 j} \frac{\partial u_{j}}{\partial \xi_{k}} \frac{\partial \xi_{k}}{\partial x_{1}}, \\
\epsilon_{22} & =A_{2 j} \frac{\partial u_{j}}{\partial \xi_{k}} \frac{\partial \xi_{k}}{\partial x_{2}}, \\
\epsilon_{12} & =\frac{1}{2}\left(\epsilon_{11}+\epsilon_{2}\right) .
\end{aligned}
$$

Então, finalmente, pode se transferir as tensões das coordenadas locais para o sistema cartesiano global:

$$
\sigma_{i j}=\mathbf{A}_{k i} \mathbf{A}_{n j} \sigma_{k n} .
$$




\section{RESULTADOS NUMÉRICOS}

\subsection{Problemas potencias}

Nessa seção, para avaliar a formulação isogeométrica potencial, dois problemas bidimensionais e três tridimensionais são apresentados. Mais exemplos, com muitos graus de liberdade, serão apresentados numa seção posterior de análise dos efeitos da ACA. Nos problemas bidimensionais, um método padrão com elementos constantes e usando integração analítica será usado como comparação. Nos casos tridimensionais, será comparado com os resultados provenientes do programa encontrado em Liu (2009), que, por sua vez, faz uso de elementos triangulares constantes e do fast multipole. A raiz do valor quadrático médio (RMS) do erro normalizado é usado como medida de erro em alguns problemas. Esse medidor é dado por:

$$
e_{r m s}=\frac{\sqrt{\frac{\sum_{t=1}^{n}\left(y_{t}-y_{a}\right)^{2}}{n}}}{y_{\max }-y_{\min }} .
$$

onde $y_{t}$ é o valor da da variável calculada numericamente no nó $t, y_{a}$ é o valor da variável calculada analiticamente o $t, n$ é o número de nós, $y_{\max }$ e $y_{\min }$ são os valores máximos e mínimos da variável $y_{a}$. Esse é um bom medidor da acurácia do método e, por ser normalizado e não depender de escala, pode ser usado para comparar problemas distintos.

\subsubsection{Condução de calor em um cilindro}

O primeiro exemplo é o de um problema de condução de calor em um cilindro que é modelado como um problema 2D conforme a figura 6.1. Esse problema tem solução analítica conhecida que será usada para verificar os resultados obtidos pelo método dos elementos de contorno isogeométricos. A temperatura é conhecida no contorno interno $S_{i}$ e o fluxo no contorno externo $S_{e}$. A solução analítica para a temperatura desse problema é dado por (BRAGA, 2013):

$$
T(r)=T_{i}+q_{e} r_{e} \log \left(\frac{r}{r_{i}}\right)
$$

e para o fluxo por:

$$
q(r)=q_{e} \frac{r_{e}}{r}
$$

onde $T_{i}$ e $q_{e}$ são a temperatura e fluxo nos contornos internos e externos, respectivamente.

Para esse problema, o raio interno é definido como $1 \mathrm{~m}$, o raio externo como $2 \mathrm{~m}$, a 


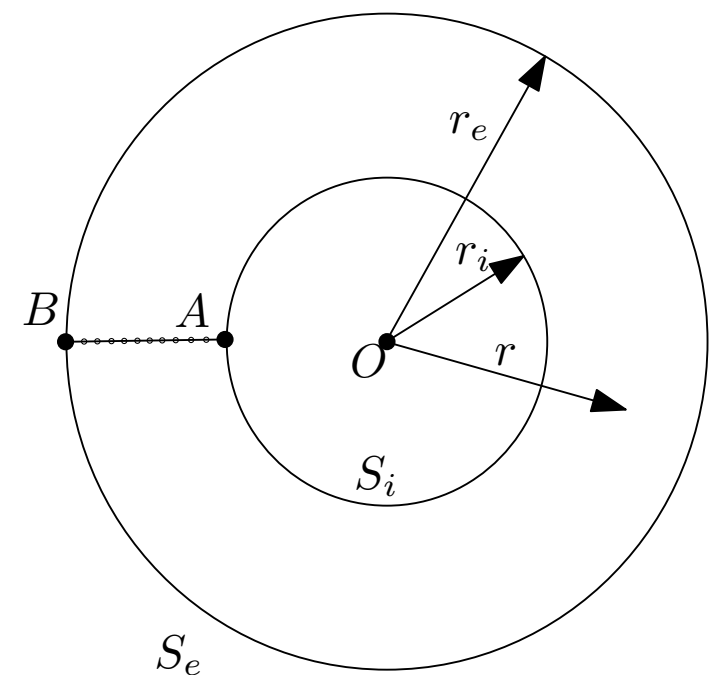

Figura 6.1: Problema potencial simples em uma região anelar

temperatura interna como $100 \mathrm{~K}$, o fluxo de calor externo como $200 \mathrm{Wm}^{-2}$ e a condutividade térmica como $1 \mathrm{Wm}^{-1} \mathrm{~K}^{-1}$. Temos como solução analítica uma temperatura externa de $377,258872 \mathrm{~K}$ e fluxo interno de $-400 \mathrm{Wm}^{-2}$. O contorno externo e o interno são discretizado com o mesmo número de elementos. Nas figuras 6.2 e 6.3 estão os erros percentuais da temperatura no ponto $B$ e o fluxo no ponto $A$ encontrados com elementos constantes e elementos quadráticos isogeométricos.

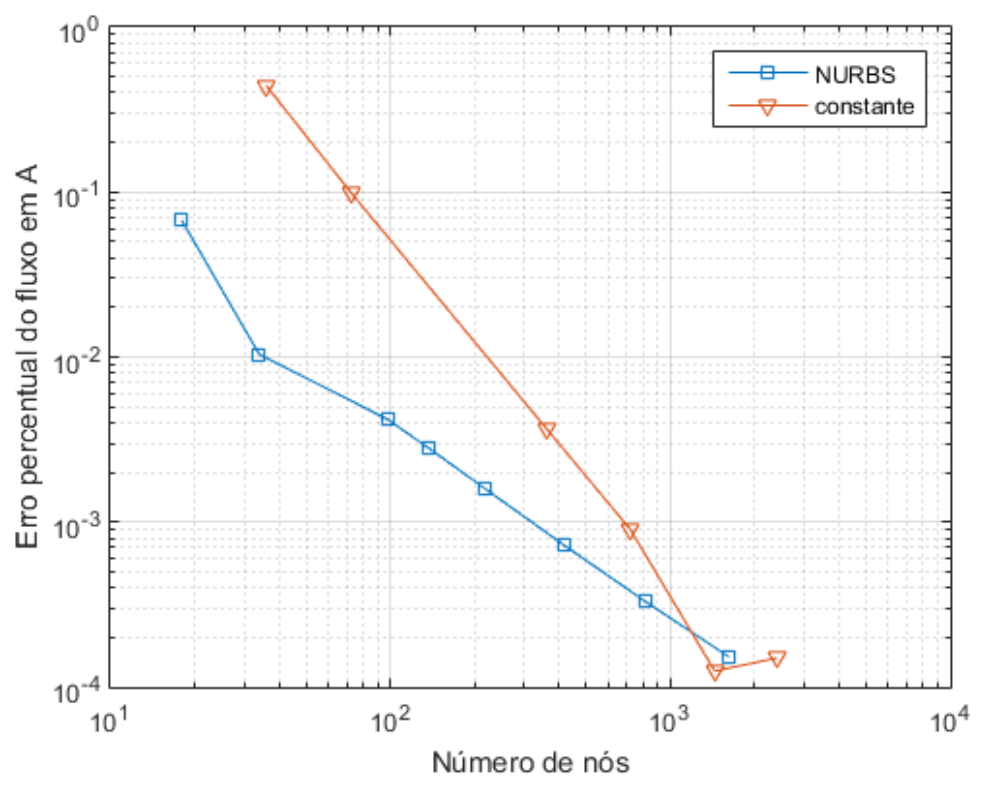

Figura 6.2: Resultado do fluxo no ponto A

Os dois tipos elementos convergem rapidamente para a solução exata. A discretização isogeométrica tem uma resposta melhor com poucos elementos e os elementos constantes tem uma convergência mais rápida. Como as variáveis do contorno são todas constantes, a maior diferença entre os métodos seria a representação da geome- 


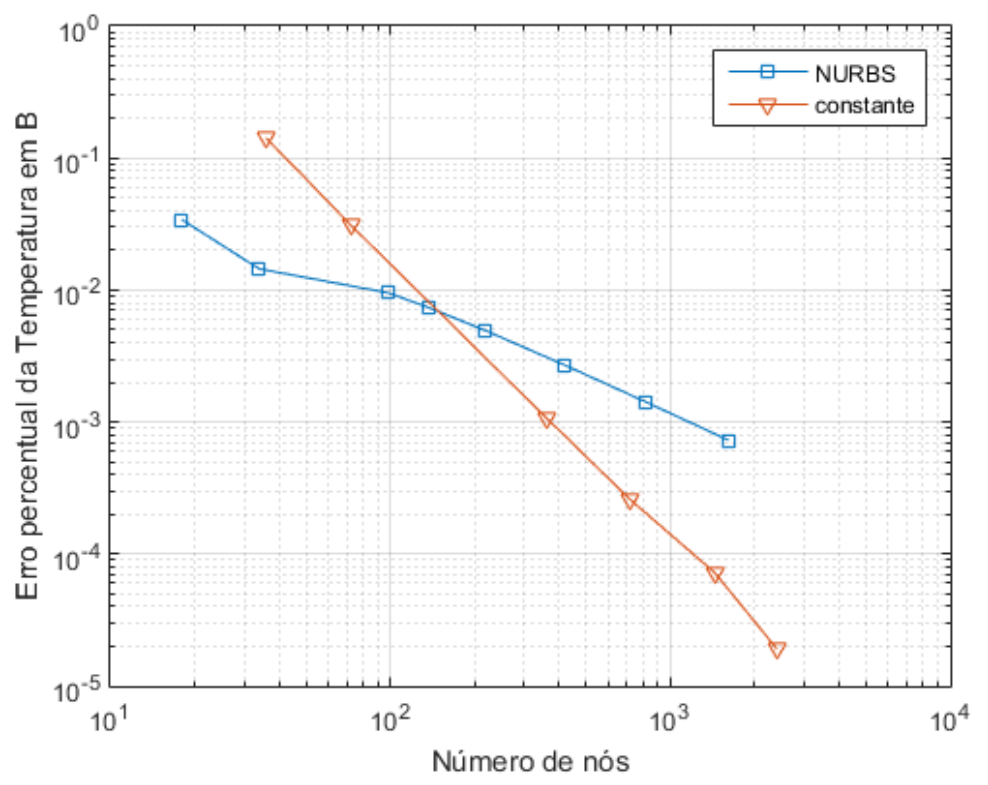

Figura 6.3: Resultado da temperatura no ponto B

tria. No caso isogeométrico, mesmo a discretização mais pobre representa exatamente a geometria enquanto isso não ocorre nos elementos constantes.

Para verificar as aproximações nos pontos internos, a temperatura é calculada em 10 pontos uniformemente distribuídos ao longo do segmento $A B$. O erro RMS (root mean square) e o erro máximo nesses pontos são calculados e mostrados na figura 6.4 .

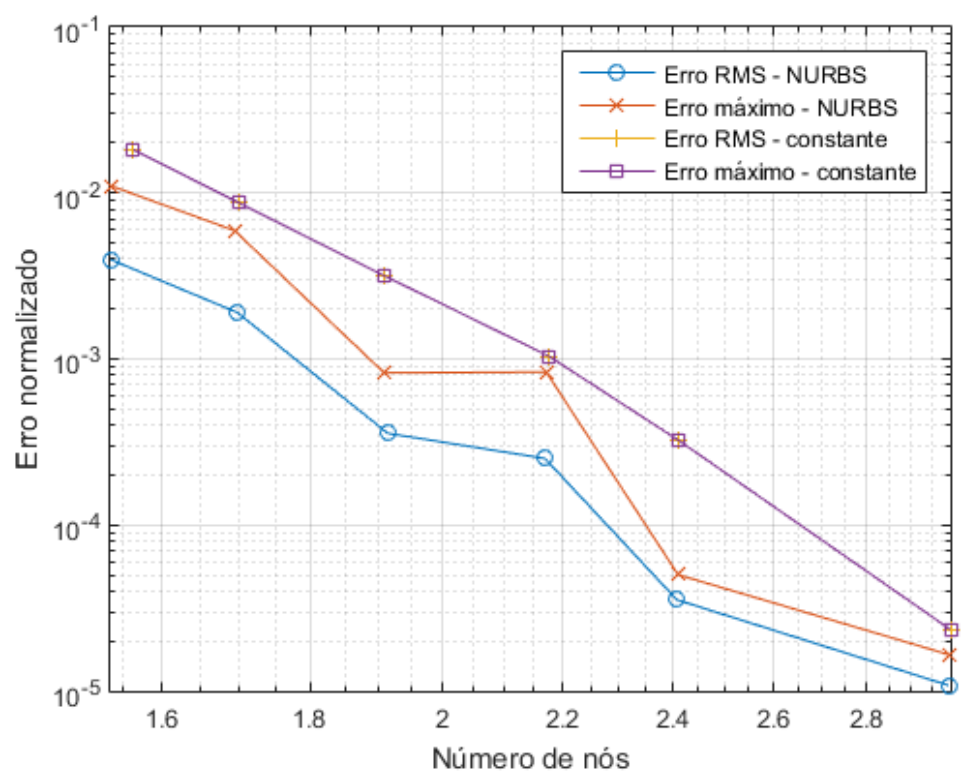

Figura 6.4: Erros obtidos ao longo de $\mathrm{AB}$

O erro obtido no método isogeométrico se mostra inferior ao erro obtido com elementos constante mesmo para uma quantidade de nós que o erro no contorno obtido 
com elementos constantes é menor. Isso pode ser explicado pela geometria exatamente representada no método isogeométrico.

Braga (2013) não observa grande diferença, para problemas potenciais, entre os elementos constantes, lineares e quadráticos. Portanto, a comparação com elementos constantes com integração analítica já é suficiente para comparar o método isogeométrico com o método padrão.

\subsubsection{Placa retangular}

O problema agora consiste em uma placa retangular como na figura 6.5 .

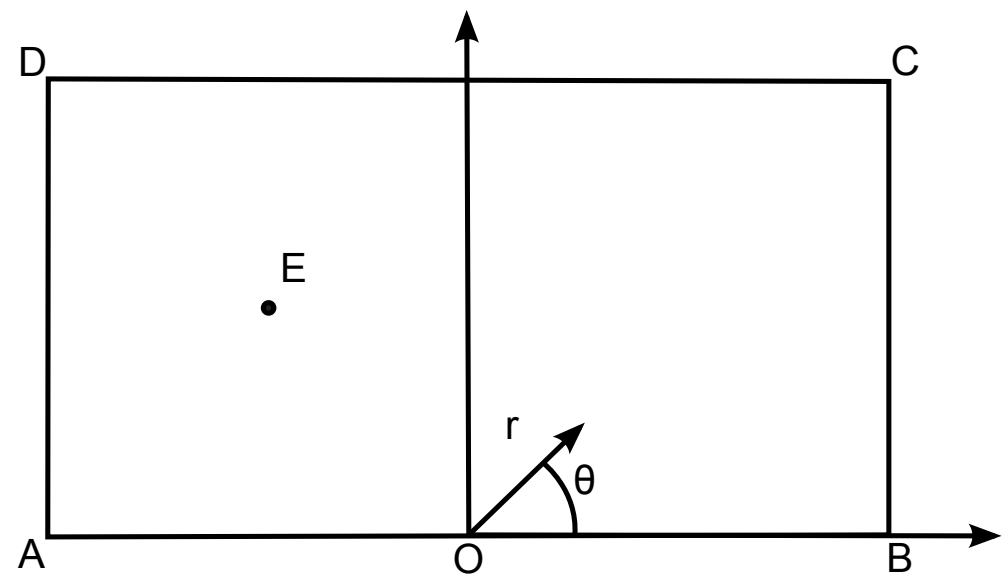

Figura 6.5: Placa retangular

Onde as condições de contorno são:

$$
\begin{gathered}
q=-\frac{1}{2 \sqrt{r}}\left(\cos \frac{\theta}{2} \cos \theta+\sin \frac{\theta}{2} \sin \theta\right) \text { em } B C, \\
q=-\frac{1}{2 \sqrt{r}}\left(\cos \frac{\theta}{2} \cos \theta-\sin \frac{\theta}{2} \sin \theta\right) \text { em } C D, \\
q=\frac{1}{2 \sqrt{r}}\left(\cos \frac{\theta}{2} \cos \theta+\sin \frac{\theta}{2} \sin \theta\right) \text { em } D A, \\
T=0 \text { em } A O
\end{gathered}
$$

$\mathrm{e}$

$$
q=0 \text { em } O B
$$

A solução analítica desse problema é dada por:

$$
\begin{gathered}
u=\sqrt{r} \cos \frac{\theta}{2} \\
q_{x}=\frac{\cos \frac{\theta}{2}}{2 \sqrt{r}}
\end{gathered}
$$


$\mathrm{e}$

$$
q_{y}=\frac{\sin \frac{\theta}{2}}{2 \sqrt{r}}
$$

Na figura 6.6 pode-se observar uma boa concordância entre o resultado obtido, com 60 elementos isogeométricos, e a solução analítica.
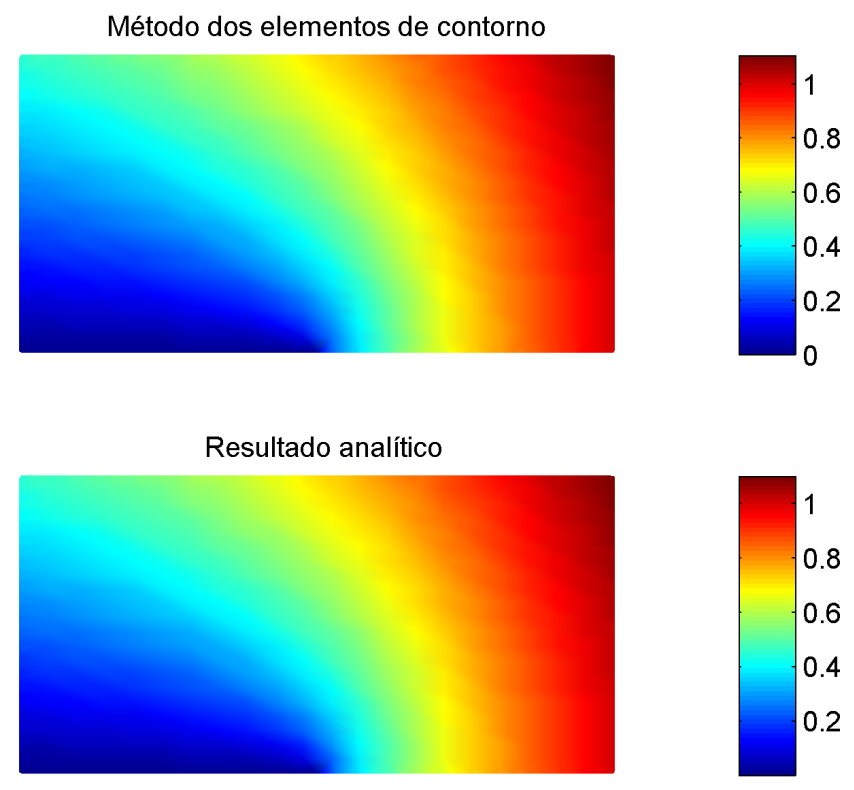

Figura 6.6: Temperatura obtida para a placa retangular

Para analisar com maior detalhes, nas figuras 6.7 e 6.8 observa-se o erro percentual da temperatura no ponto $E$ para elementos isogeométricos de diferentes ordens e elementos constantes padrões. Na figura 6.7. o método proposto na seção 5.6 para a imposição das condições de contorno é utilizado, enquanto na figura 6.8 não há o cuidado de fazê-lo. Fica nítida a influência da transformação: os resultados obtidos com o método proposto são muito superiores à alternativa. Esse problema é um ótimo exemplo uma vez que as condições de contorno impostas não são constantes. São, na verdade, bastante complexas, contendo inclusive uma singularidade no ponto $O$. Enquanto isso a geometria do problema é muito simples, sendo representada exatamente por elementos de ordem qualquer. Mesmo na formulação de contorno padrão, não há erros devido a aproximação da geometria.

Os resultados encontrados na figura 6.7 mostram uma melhora na convergência a medida que se aumenta a ordem da curva. Pode-se observar que mesmo o elemento isogeométrico de mais baixa ordem, ordem 2, teve uma resposta melhor que os elementos constantes padrões.

A figura 6.9 mostra o tempo de montagem das matrizes em relação ao número de 


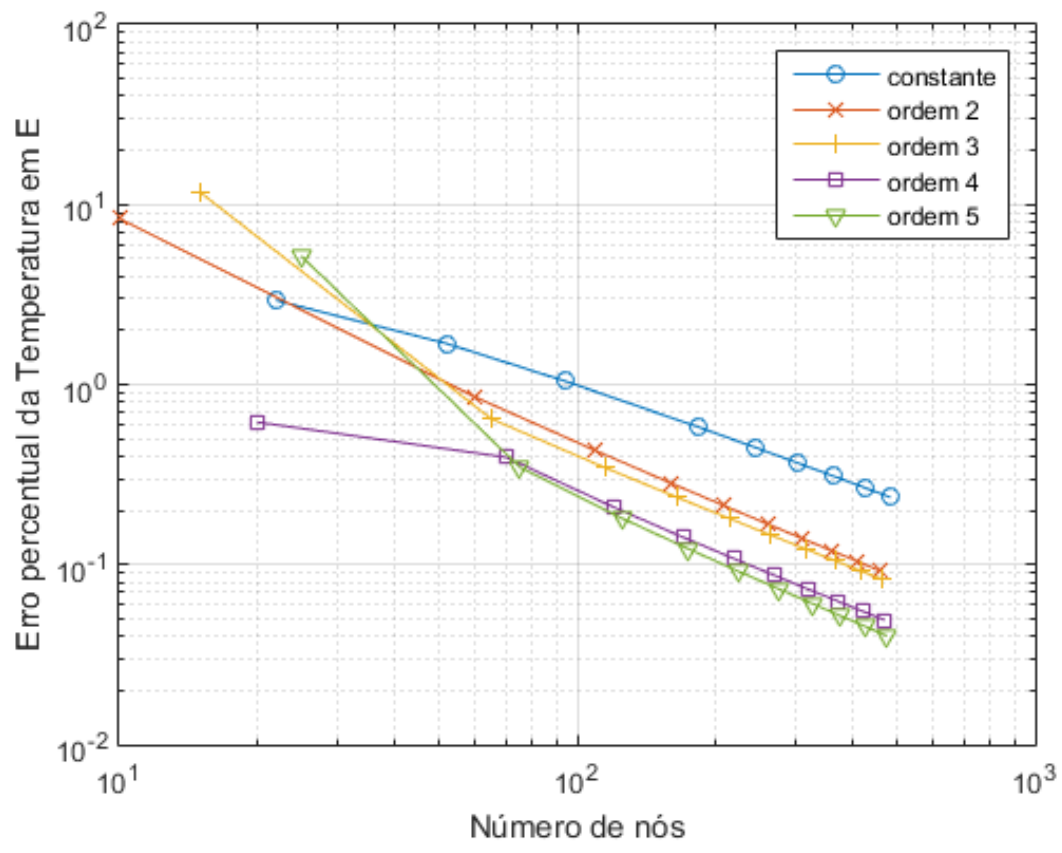

Figura 6.7: Convergência para a solução analítica do ponto E - método proposto

nós. O tempo foi normalizado pela menor medida encontrada. Como as distintas curvas são paralelas no gráfico log-log, fica claro que ao aumentar a ordem, a complexidade do algorítmo não é aumentada. Com o aumento da ordem da NURBS, o processo fica mais demorado e o tempo gasto é multiplicado por uma constante. Isso pode ser observado claramente na figura 6.10 onde o tempo está relacionado com distintas ordens mas mantendo o número de nós constantes. 


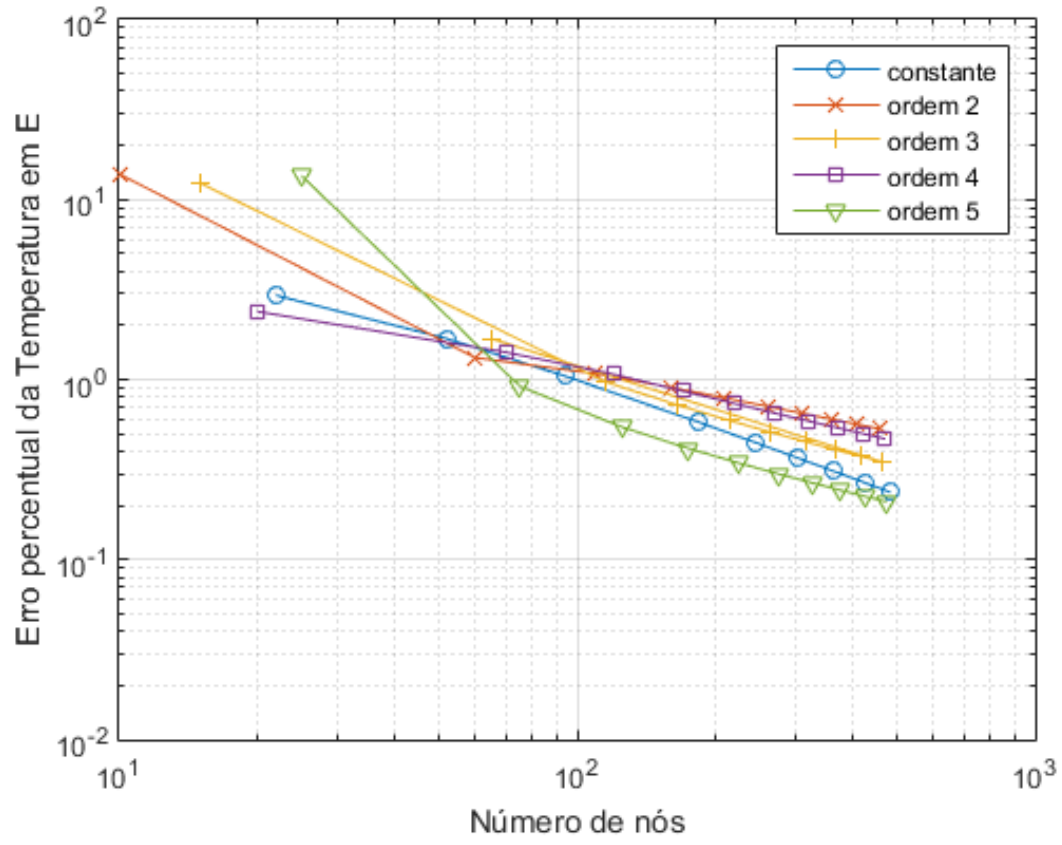

Figura 6.8: Convergência para a solução analítica do ponto E - sem a utilização do método proposto

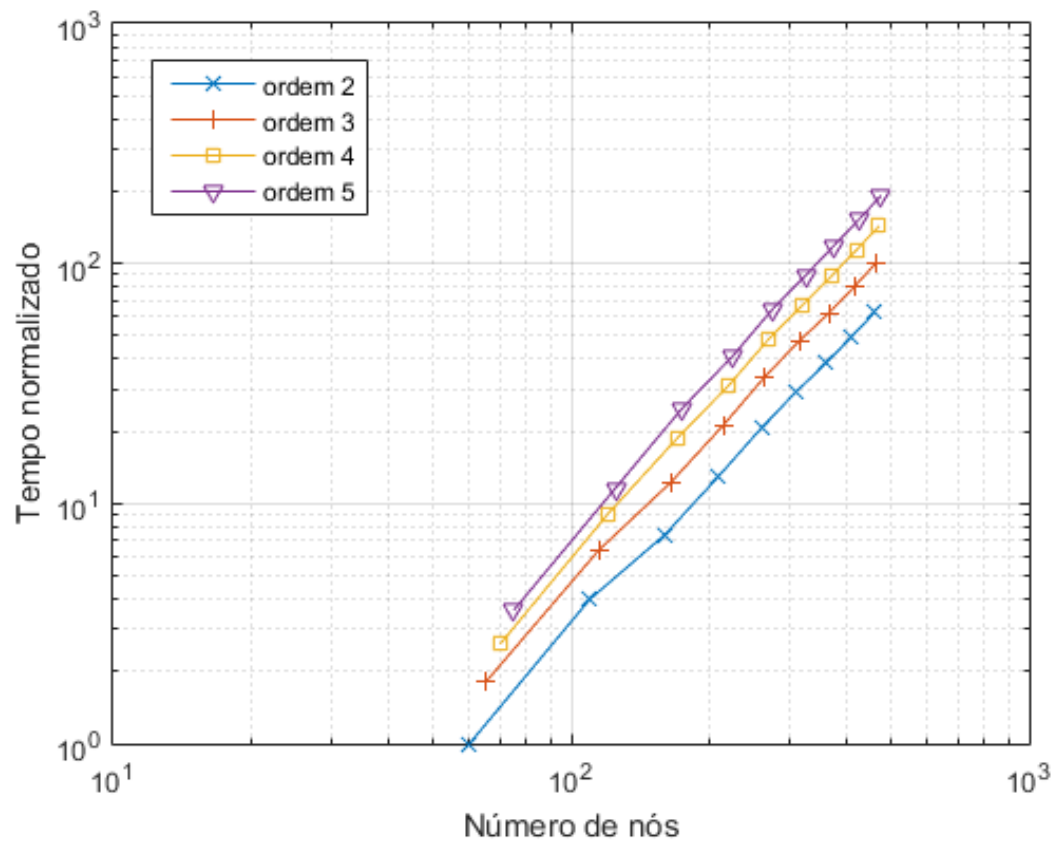

Figura 6.9: Tempo gasto na montagem da matriz em relação ao número de nós 


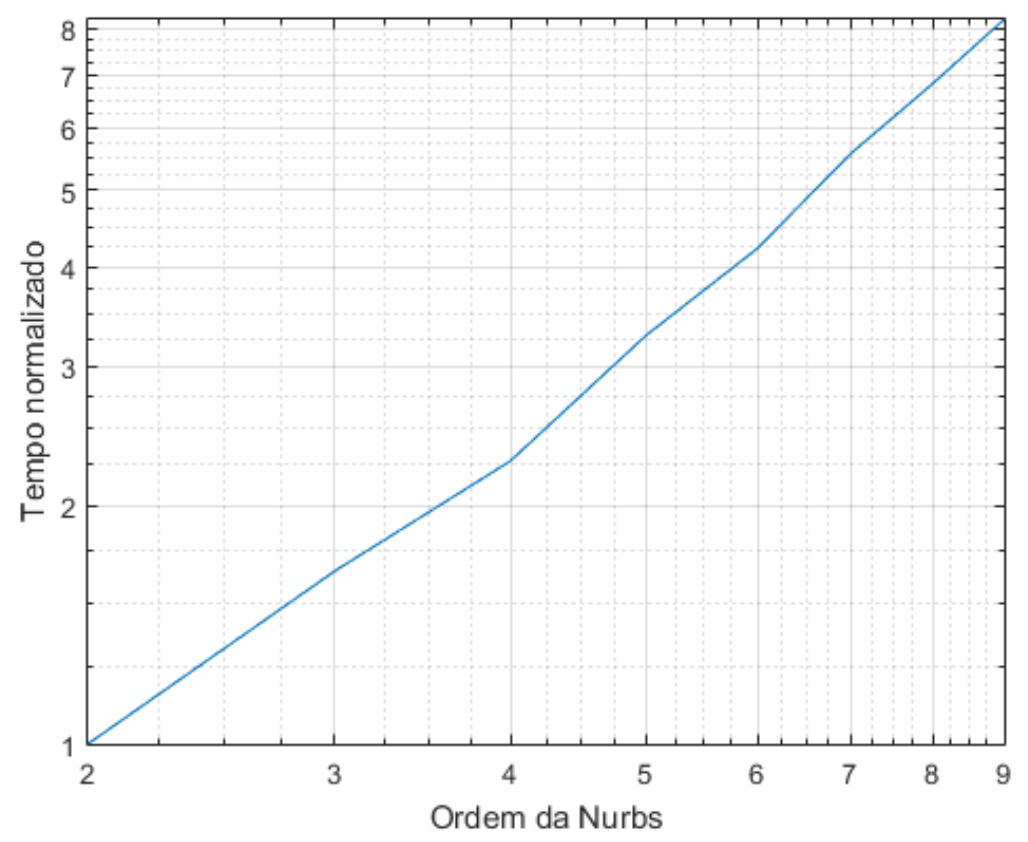

Figura 6.10: Tempo gasto na montagem da matriz em relação a ordem da NURBS 


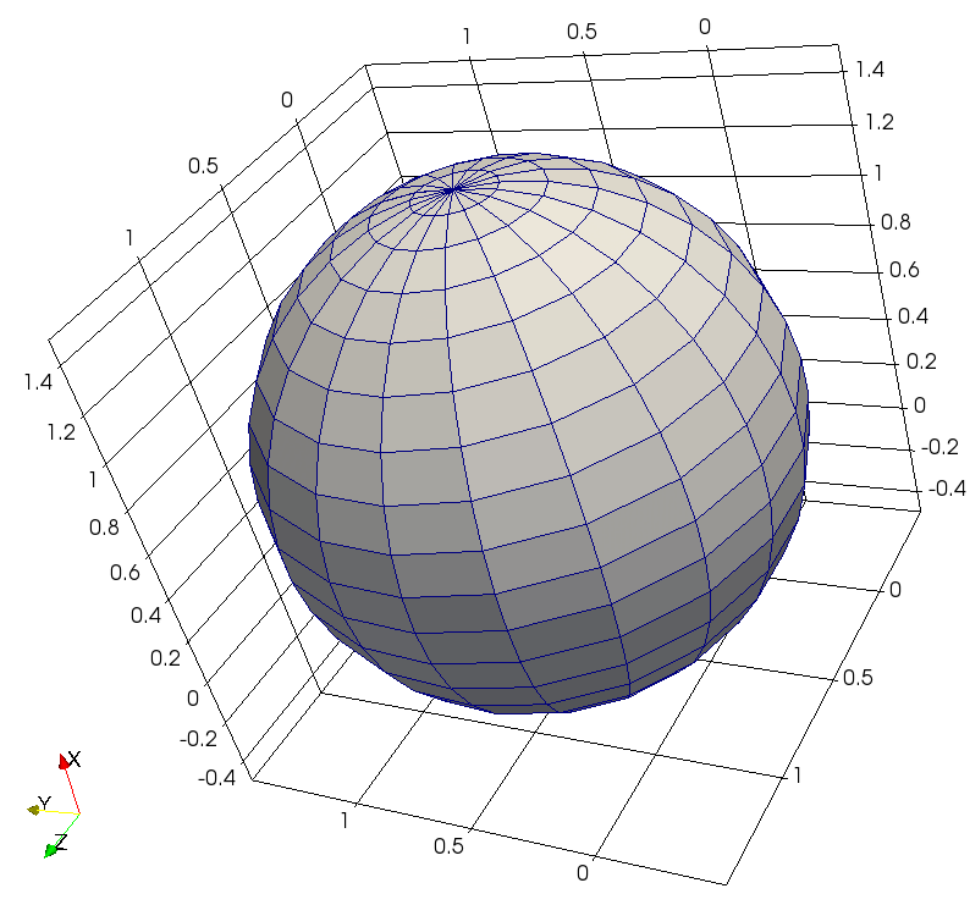

Figura 6.11: Esfera discretizada pelas NURBS

\subsubsection{Esfera}

Nessa seção, analisa-se o problema apresentado por Zhang, Gong e Gao (2015). O problema consiste em uma estrutura esférica tri-dimensional de raio 1 e centro $(0,5 ; 0,5 ; 0,5)$ conforme apresentado na figura 6.11. A temperatura prescrita no contorno é:

$$
u=x^{2}-y^{2}+2 x+z
$$

e a solução analítica do fluxo é dada por $-k \mathbf{n} \nabla u$ onde:

$$
\nabla u=\left[\begin{array}{lll}
2 x+2 & -2 y & 1
\end{array}\right]
$$

Na tabela 6.1, compara-se o erro RMS normalizado para elementos triangulares constantes e para elementos isogeométricos de ordem 3. Nessa comparação fica claro que o desempenho dos elementos isogeométricos é muito superior ao dos elementos constantes. No entanto, a discretização da esfera gera dois polos onde acontece uma concentração dos pontos de controle, como pode ser observado na figura 6.11. Essa concentração exige uma atenção especial no tratamento das singularidades. As figuras 6.12 e 6.13 mostram o comportamento da solução fundamental, no espaço paramétrico, para um ponto fonte fora do polo e para um ponto fonte igual a um dos polos. Quando o 
Tabela 6.1: Erro RMS normalizado para o exemplo da esfera

\begin{tabular}{|c|c|c|c|}
\hline \multicolumn{2}{|c|}{ Elementos constantes } & \multicolumn{2}{c|}{ Elementos isogeométricos - ordem 3 } \\
\hline Nós & Erro RMS normalizado & Nós & Erro RMS normalizado \\
\hline 320 & 0,017847 & 45 & 0,0110208 \\
1280 & 0,011417 & 91 & 0,00231124 \\
5120 & 0,010104 & 153 & 0,00151761 \\
20480 & 0,0099437 & & \\
\hline
\end{tabular}

ponto fonte se aproxima dos polos a singularidade que deveria ser pontual se distribui ao longo de uma das direções da integração, tornando, assim, todas as integrais, próximas aos polos, quase singulares. O tratamento das integrais singulares também sofrem com a distorção dos elementos (HAYAMI; MATSUMOTO, 1994) sendo necessários mais de 500 pontos de integração nos polos para se obter um erro de integração menor que $10^{-6}$.

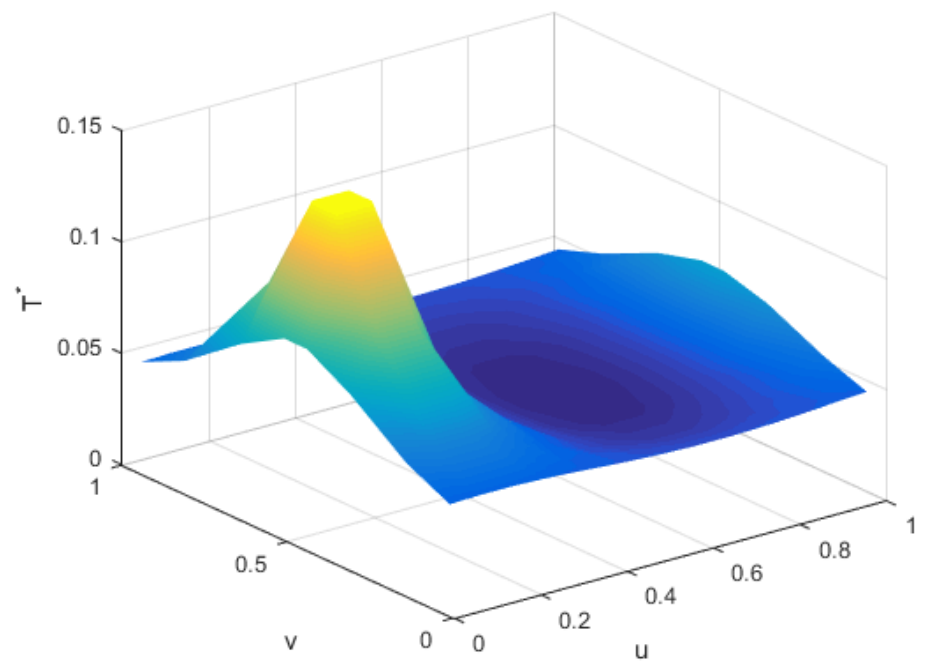

Figura 6.12: Solução fundamental para pontos fonte $=(0,51,50,5)$

\subsubsection{Toroide}

Nesse exemplo, apresentado em Zhang, Gong e Gao (2015), um toroide de raio exterior e raio interior iguais a $R=2$ e $r=1$, respectivamente, é analisado sobre uma temperatura no contorno igual a:

$$
u=\frac{x^{2}}{2}+\frac{y^{2}}{2}-z^{2}+5 x+5 y+5 z
$$

e a solução analítica do fluxo é dada por $-k \mathbf{n} \nabla u$, onde: 


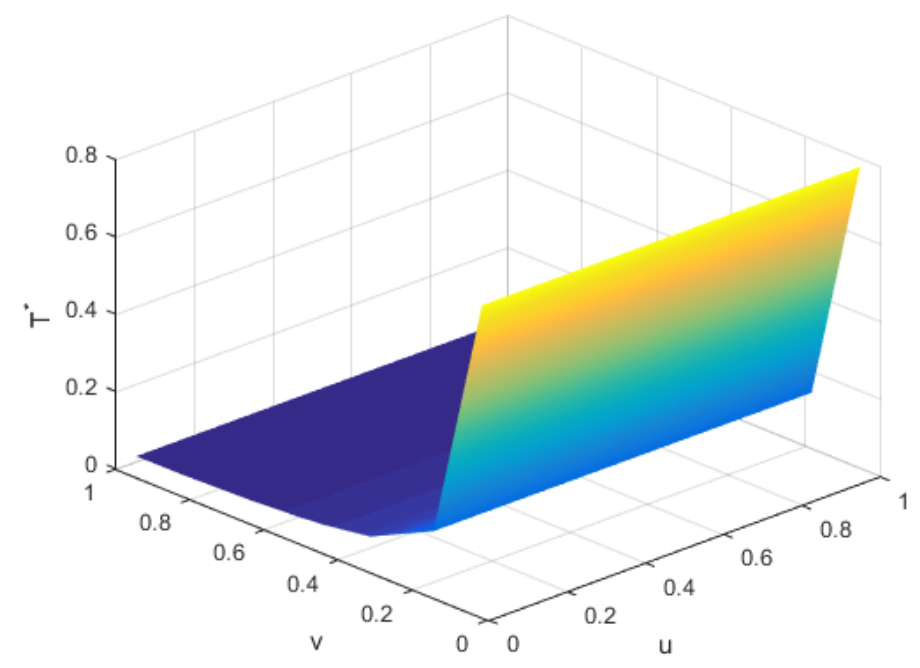

Figura 6.13: Solução fundamental para distintos pontos fonte $=\left(\begin{array}{ll}1,5 & 0,5 \\ 0,5\end{array}\right)$

Tabela 6.2: Erro RMS normalizado para o exemplo do toroide

\begin{tabular}{|c|c|c|c|}
\hline \multicolumn{2}{|c|}{ Elementos constantes } & \multicolumn{2}{c|}{ Elementos isogeométricos - ordem 3 } \\
\hline Nós & Erro RMS normalizado & Nós & Erro RMS normalizado \\
\hline 1792 & 0,0086708 & 81 & 0,0176615 \\
28224 & 0,0098135 & 169 & 0,0027632 \\
& & 441 & 0,000568215 \\
\hline
\end{tabular}

$$
\nabla u=\left[\begin{array}{lll}
x+5 & y+5 & -2 z+5
\end{array}\right]
$$

Na figura 6.14 pode-se observar o toroide analisado e o fluxo obtido com 81 pontos de controle. Pode-se observar que não existe concentração de nós nessa geometria, sendo o tratamento das singularidades mais simples que o caso da esfera.

A tabela 6.2, onde estão os erros RMS dos pontos de colocação, mostra uma clara vantagem do método isogeométrico quando comparado ao elemento constante padrão atingindo uma alta precisão com uma quantidade muito menor de nós. Em Zhang, Gong e Gao (2015) pode-se comparar com elementos quadráticos padrões, onde com 1600 nós se alcança um erro de $10^{-3}$. Portanto, mesmo comparando com elementos de mesma ordem o método isogeométrico apresenta vantagem sobre o padrão.

\subsubsection{Cilindro vazado}

Estuda-se o caso 3.1.1, com as mesma propriedades usadas na seção anterior, como um corpo tridimensional. Na figura 6.15 pode-se observar o cilindro analisado e a temperatura obtida com 700 pontos de controle. 


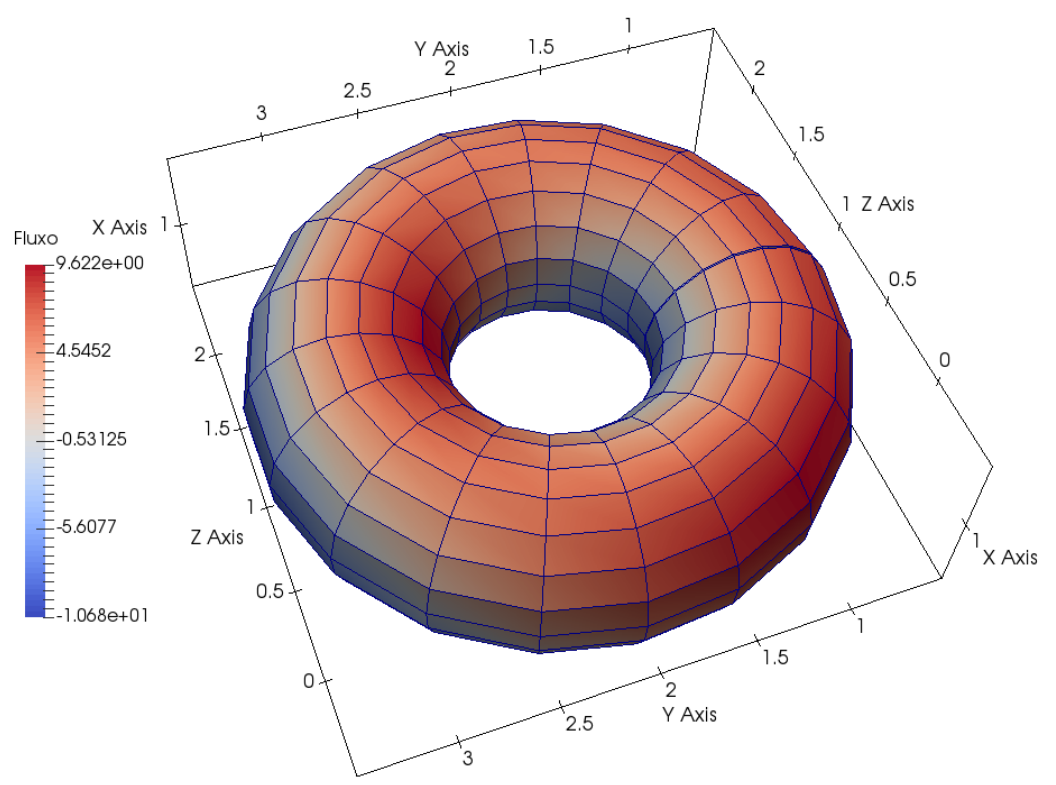

Figura 6.14: Toroide discretizado pelas NURBS

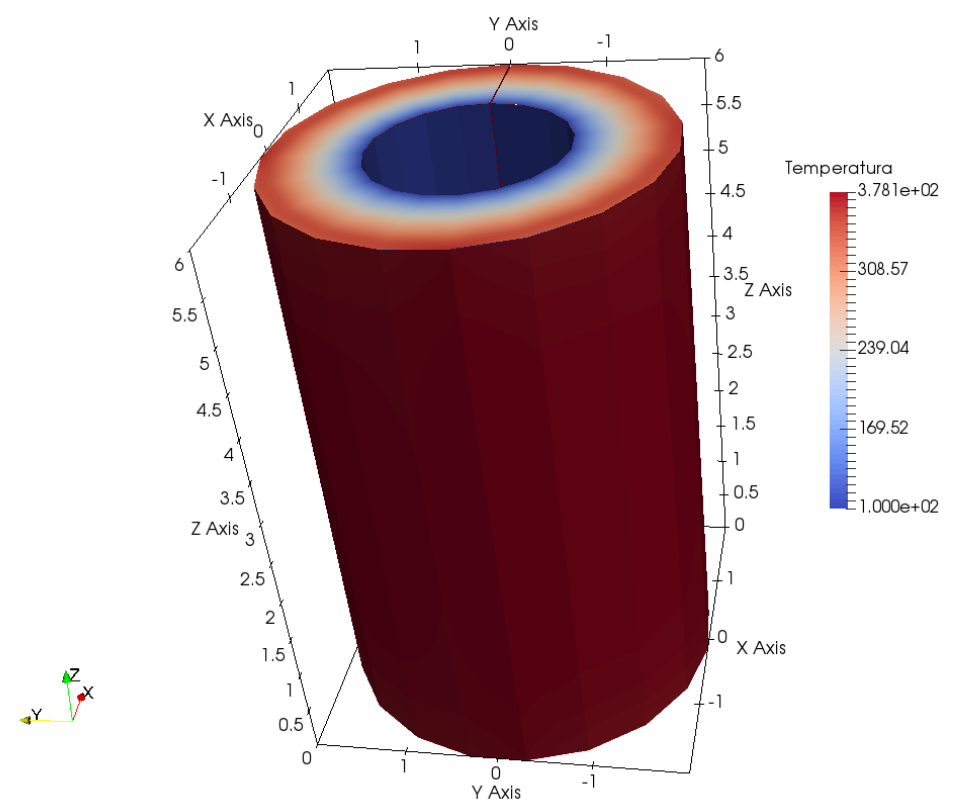

Figura 6.15: Cilindro discretizado pelas NURBS 
Tabela 6.3: Erro RMS normalizado para o exemplo do cilindro

\begin{tabular}{|c|c|c|c|}
\hline \multicolumn{4}{|c|}{ Temperatura externa } \\
\hline \multicolumn{2}{|c|}{ Elementos constantes } & Elementos isogeométricos - ordem 3 \\
\hline Nós & Erro & Nós & Erro \\
\hline 1506 & 0,0673 & 108 & 0,0085888 \\
4412 & 0,0306 & 700 & 0,0035181 \\
12688 & 0,0211 & & Fluxo interno \\
\hline \multicolumn{3}{|c|}{} \\
\hline \multicolumn{3}{|c|}{ Elementos constantes } & Elementos isogeométricos - ordem 3 \\
\hline Nós & Erro & Nós & Erro \\
\hline 1506 & 0,1899 & 108 & 0,0308377 \\
4412 & 0,1822 & 700 & 0,0109930 \\
12688 & 0,1976 & & \\
\hline
\end{tabular}

A tabela 6.3 , onde estão os erros RMS dos pontos de colocação para a temperatura externa e o fluxo interno, mostra o método tendo uma vantagem mesmo se tratando de um problema onde as condições de contorno nas faces internas e externas são constantes.

\subsubsection{Cubo}

Nos exemplos anteriores, o método isogeométrico apresentava vantagem clara na representação da geometria, sendo uma representação exata enquanto o método padrão apenas a aproximava. Por isso, esse exemplo trata de um cubo de lado 10 que será representado exatamente pelos dois métodos. A temperatura imposta é igual a:

$$
u=x^{3}+y^{3}+3 z^{3}-x z^{3}-6 y x^{2}-9 z y^{2}
$$

e a solução analítica do fluxo é dada por $-k \mathbf{n} \nabla u$, onde:

$$
\nabla u=\left[\begin{array}{lll}
3 x^{2}-3 z^{2}-12 x y & -6 x^{2}+6 y^{2}-18 y z & -9 y^{2}+9 z^{2}-6 x z
\end{array}\right] .
$$

Na figura 6.16 pode-se observar o cubo analisado e o fluxo obtido com 216 pontos de controle. Nesse problema, como existe uma descontinuidade da normal entre as faces, o fluxo não é continuo entre as faces e o método proposto se mostrou eficaz na representação desse campo descontínuo.

A tabela 6.4. onde estão os erros RMS dos pontos de colocação, mostra, mais uma vez, uma clara vantagem do método isogeométrico quando comparado ao elemento de contorno padrão. O aumento da ordem do elemento se mostrou vantajosa nesse problema, no entanto, mesmo o elemento de ordem 2 teve uma convergência muito 


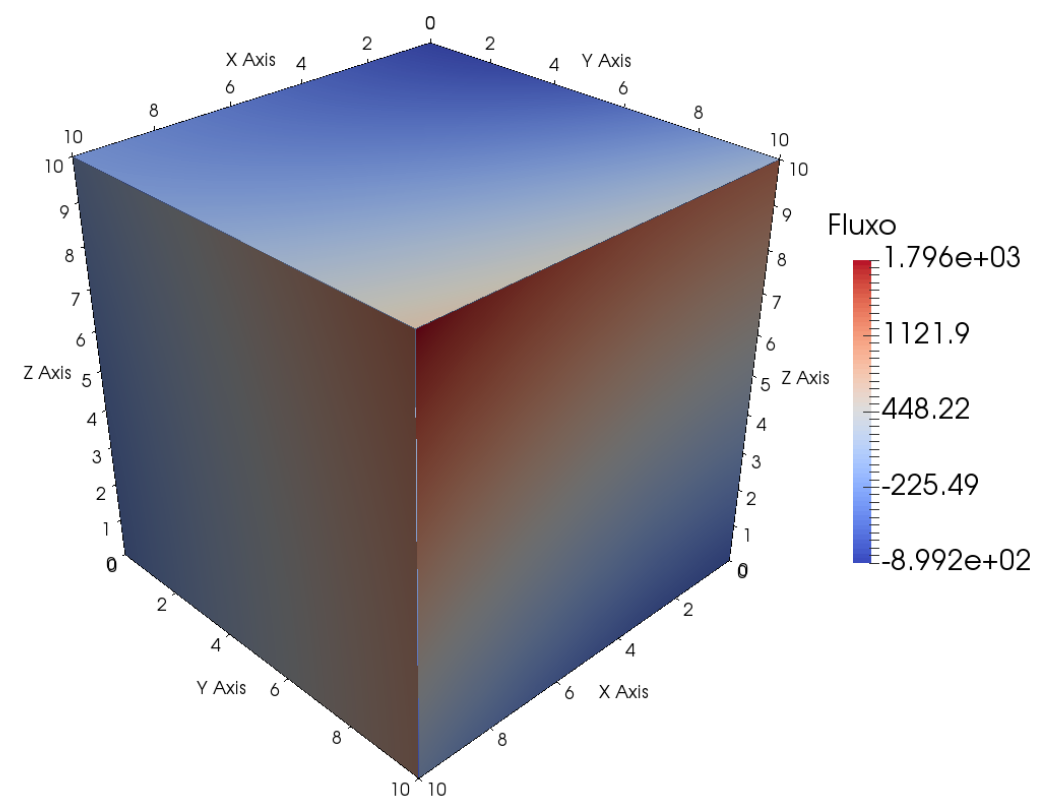

Figura 6.16: Cubo discretizado pelas NURBS

Tabela 6.4: Erro RMS normalizado para o exemplo do cubo

\begin{tabular}{|c|c|c|c|c|c|}
\hline \multicolumn{2}{|c|}{$\begin{array}{c}\text { Elementos } \\
\text { constantes }\end{array}$} & \multicolumn{2}{c|}{$\begin{array}{c}\text { Elementos isogeométricos } \\
\text { ordem } 2\end{array}$} & \multicolumn{2}{c|}{$\begin{array}{c}\text { Elementos isogeométricos } \\
\text { ordem 3 }\end{array}$} \\
\hline Nós & Erro & Nós & Erro & Nós & Erro \\
\hline 1266 & 0,017333 & 24 & 0,0300352 & 54 & 0,0063108 \\
5394 & 0,012088 & 96 & 0,00748032 & 96 & 0,00171195 \\
21828 & 0,008872 & 216 & 0,00401579 & 150 & 0,000513754 \\
\hline
\end{tabular}


rápida quando comparado ao método padrão.

\subsection{Problemas elásticos}

Esta seção apresenta os resultados obtidos para problemas de elasticidade plana (tensão plana). As respostas são comparadas com soluções analíticas sempre que disponíveis e com os resultados obtidos utilizando a formulação padrão do MEC. No caso bidimensional o método é comparado com elementos constantes e quadráticos enquanto os casos tridimensionais serão comparados com o elemento triangular constante e elementos quadráticos quadrilaterais.

\subsubsection{Viga bidimensional}

Nessa seção a viga bidimensional é analisada. São tratadas vigas engastadas de duas distintas razões de aspecto: 4:1, ou seja, 4 unidades de comprimento na horizontal para 1 unidade na vertical e 10:1 e com carregamentos distribuídos e concentrados. Na figura 6.17, as diferentes configurações podem ser observadas.

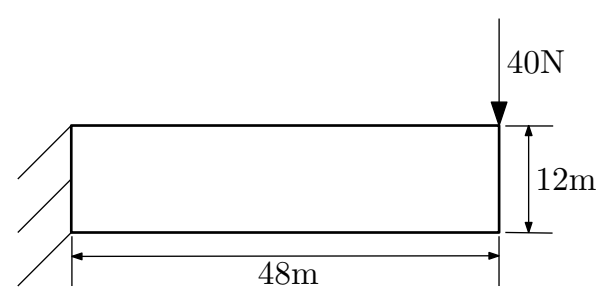

(a) Caso 1

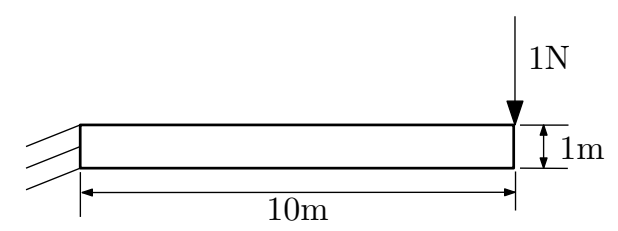

(c) Caso 3

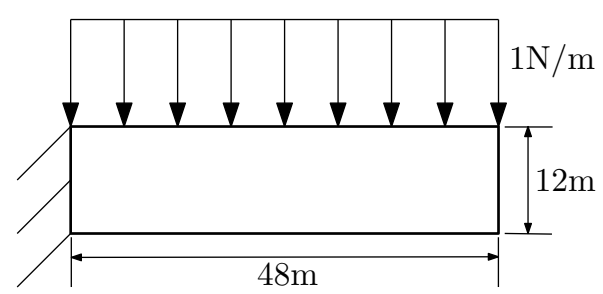

(b) Caso 2

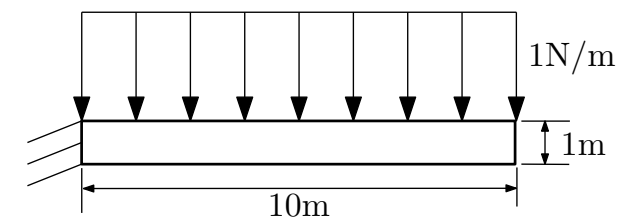

(d) Caso 4

Figura 6.17: Diferentes configurações de viga

A solução analítica é conhecida e é apresentada em Timoshenko (1934). Para carregamento concentrado a solução é dada por:

$$
u_{y}=\frac{P}{6 E I}\left[\frac{b^{2}}{4}(5 \mu+4) L+2 L^{3}\right] \text {, }
$$

e para carregamento distribuído por:

$$
u_{y}=\frac{q L^{4}}{8 E I}
$$


Utilizando como propriedades do problema, $E=30 \mathrm{kPa}, v=0.25$ encontra-se uma deflexão máxima de 0,35533 m para o caso 1, 0,1536 m para o caso 2, 0,13421 m para o caso 3 e $0,5 \mathrm{~m}$ para o caso 4 .

As tabelas 6.5 e 6.6 mostram os resultados obtidos incluindo o erro percentual relativo. Ao analisar as tabelas, fica evidente que o desempenho dos elementos constantes é bem inferior aos demais. Enquanto isso, os elementos isogeométricos, de diferentes ordens, tem uma convergência muito parecida com a dos elementos quadráticos contínuos.

Ao trocar do carregamento concentrado pelo carregamento distribuído, há um aumento do erro que pode ser explicado pelo fato da solução analítica usada não levar em conta os efeitos de cisalhamento.

O MEC converge para um valor distinto da solução analítica devido a diferença na maneira de se impor as condições de contorno para este problema, conforme explicado por Costa (2015) Uma comparação mais precisa pode ser obtida utilizando exatamente as mesmas condições de contorno usadas na obtenção da solução analítica de Timoshenko.

\subsubsection{Placa com furo central}

Nessa seção, será considerado o exemplo de um furo circular numa placa quadrada de espessura constante, como apresentado na figura 6.18. A placa analisada tem como propriedades $L=200 \mathrm{~mm}, d=20 \mathrm{~mm}, E=200 \mathrm{GPa}$ e $v=0.3$. As tensões $\sigma_{1}$ e $\sigma_{2}$ são variadas de modo a gerar três casos distintos: tração uniaxial, $\sigma_{1}=1 \mathrm{MPa}$ e $\sigma_{2}=0$, tração biaxial, $\sigma_{1}=\sigma_{2}=1 M P a$, e cisalhamento puro, $\sigma_{1}=1 \mathrm{MPa}$ e $\sigma_{2}=-1 \mathrm{MPa}$. Pilkey (1993) apresenta valores dos fatores de concentração de tensão para esses três casos, assumindo uma placa infinita. Os valores encontrados são: tração uniaxial: $K_{t}=3$, tração biaxial: $K_{t}=2$, e cisalhamento puro: $K_{t}=4$.

Na tabela 6.7 está os fatores calculados pelo MEC e o erro relativo comparando com o fator teórico. Tanto a formulação padrão quanto a isogeométrica tem uma convergência rápida. Mesmo tendo, na geometria, um círculo aproximado, o elemento quadrático padrão teve desempenho ligeiramente melhor que o isogeométrico.

\subsubsection{Cubo}

Esse exemplo trata de um cubo de lado $10 \mathrm{~m}$ sob tração, conforme figura 6.19. Esse exemplo simples foi escolhido para tentar tirar todas as vantagens do método isogeométrico: uma geometria que é representada exatamente por elementos constantes e condições de contorno constantes ou lineares que podem ser obtidas facilmente por elementos constantes. Nesse exemplo o método padrão, com 300 elementos constantes, 
Tabela 6.5: Viga de razão 4:1

\begin{tabular}{|c|c|c|c|c|}
\hline Nós & Deflexão máxima (m) & Erro relativo & Deflexão máxima $(\mathrm{m})$ & Erro relativo \\
\hline & \multicolumn{2}{|c|}{ Carga concentrada } & \multicolumn{2}{|c|}{ Carga distribuída } \\
\hline \multicolumn{5}{|c|}{ Elementos isogeométricos - ordem 2} \\
\hline 50 & 0,366435 & $3,12539 \%$ & 0,170123 & $7,0427 \%$ \\
\hline 92 & 0,365078 & $2,74341 \%$ & 0,169045 & $6,3644 \%$ \\
\hline 176 & 0,364316 & $2,52889 \%$ & 0,167712 & $5,5257 \%$ \\
\hline 344 & 0,364771 & $2,65689 \%$ & 0,167407 & $5,3338 \%$ \\
\hline 680 & 0,364763 & $2,65469 \%$ & 0,167222 & $5,2174 \%$ \\
\hline \multicolumn{5}{|c|}{ Elementos isogeométricos - ordem 3} \\
\hline 54 & 0,359284 & $1,11271 \%$ & 0,166291 & $4,6316 \%$ \\
\hline 96 & 0,364892 & $2,69106 \%$ & 0,167095 & $5,1375 \%$ \\
\hline 180 & 0,365139 & $2,76041 \%$ & 0,167736 & $5,5408 \%$ \\
\hline 348 & 0,364858 & $2,68143 \%$ & 0,167311 & $5,2734 \%$ \\
\hline 684 & 0,364818 & $2,67015 \%$ & 0,167201 & $5,2042 \%$ \\
\hline \multicolumn{5}{|c|}{ Elementos isogeométricos - ordem 4} \\
\hline 58 & 0,361469 & $1,72773 \%$ & 0,166331 & $4,6568 \%$ \\
\hline 100 & 0,364864 & $2,68323 \%$ & 0,166241 & $4,6001 \%$ \\
\hline 184 & 0,365877 & $2,9681 \%$ & 0,167895 & $5,6408 \%$ \\
\hline 352 & 0,365075 & $2,74262 \%$ & 0,167325 & $5,2822 \%$ \\
\hline 688 & 0,364933 & $2,70244 \%$ & 0,167165 & $5,1815 \%$ \\
\hline \multicolumn{5}{|c|}{ Elementos constantes } \\
\hline 100 & 0,22127 & $-35,17 \%$ & 0,10426 & $-32,12 \%$ \\
\hline 200 & 0,25737 & $-24,60 \%$ & 0,11982 & $-21,99 \%$ \\
\hline 400 & 0,27744 & $-18,72 \%$ & 0,12845 & $-16,37 \%$ \\
\hline 800 & 0,28766 & $-15,72 \%$ & 0,13285 & $-13,51 \%$ \\
\hline 1600 & 0,29268 & $-14,25 \%$ & 0,13502 & $-12,10 \%$ \\
\hline 3000 & 0,29497 & $-13,58 \%$ & 0,13608 & $-11,41 \%$ \\
\hline \multicolumn{5}{|c|}{ Elementos quadráticos contínuos } \\
\hline 88 & 0,36291 & $2,1341 \%$ & 0,16552 & $4,145 \%$ \\
\hline 176 & 0,36439 & $2,5488 \%$ & 0,16673 & $4,9095 \%$ \\
\hline 352 & 0,36492 & $2,6976 \%$ & 0,16714 & $5,1686 \%$ \\
\hline 704 & 0,3651 & $2,7488 \%$ & 0,16728 & $5,2553 \%$ \\
\hline
\end{tabular}


Tabela 6.6: Viga de razão 10:1

\begin{tabular}{|c|c|c|c|c|}
\hline Nós & Deflexão máxima $(\mathrm{m})$ & Erro relativo & Deflexão máxima (m) & Erro relativo \\
\hline \multicolumn{5}{|c|}{ Elementos isogeométricos - ordem 2} \\
\hline 50 & 0,289137 & $115,452 \%$ & 0,0907 & $118,14 \%$ \\
92 & 0,160626 & $19,6911 \%$ & 0,607601 & $21,5202 \%$ \\
176 & 0,141017 & $5,07985 \%$ & 0,529544 & $5,90875 \%$ \\
344 & 0,138011 & $2,83993 \%$ & 0,518624 & $3,72479 \%$ \\
680 & 0,137464 & $2,43246 \%$ & 0,516814 & $3,36275 \%$ \\
\hline \multicolumn{5}{|c|}{ Elementos isogeométricos - ordem 3} \\
\hline 54 & 0,135791 & $1,18527 \%$ & 0,510305 & $2,06095 \%$ \\
96 & 0,1411 & $5,14171 \%$ & 0,532488 & $6,49758 \%$ \\
180 & 0,134007 & $-0,14417 \%$ & 0,500361 & $0,0722778 \%$ \\
348 & 0,136645 & $1,82194 \%$ & 0,513148 & $2,62953 \%$ \\
684 & 0,137187 & $2,22555 \%$ & 0,515724 & $3,14471 \%$ \\
\hline \multicolumn{5}{|c|}{ Elementos isogeométricos - ordem 4 } \\
\hline 58 & 0,13543 & $0,916516 \%$ & 0,507819 & $1,56387 \%$ \\
100 & 0,138185 & $2,96927 \%$ & 0,517899 & $3,57987 \%$ \\
184 & 0,135469 & $0,945388 \%$ & 0,50728 & $1,456 \%$ \\
352 & 0,13726 & $2,28018 \%$ & 0,51589 & $3,1779 \%$ \\
688 & 0,137183 & $2,22311 \%$ & 0,5156 & $3,11996 \%$ \\
\hline \multicolumn{5}{|c|}{ Elementos constantes } \\
\hline 220 & 0,052784 & $-60,41 \%$ & 0,20583 \\
440 & 0,073217 & $-45,08 \%$ & 0,2803 & $-58,83 \%$ \\
880 & 0,089345 & $-32,99 \%$ & 0,33891 & $-43,94 \%$ \\
1760 & 0,09967 & $-25,25 \%$ & 0,37645 & $-32,22 \%$ \\
3520 & 0,10548 & $-20,89 \%$ & 0,39757 & $-20,49 \%$ \\
\hline \multicolumn{5}{|c|}{ Elementos quadráticos contínuos } \\
\hline 88 & 0,13838 & $3,1087 \%$ & 0,52092 & $3,1845 \%$ \\
176 & 0,13781 & $2,6832 \%$ & 0,5186 & $3,4978 \%$ \\
352 & 0,13756 & $2,4955 \%$ & 0,51749 \\
704 & 0,13746 & $2,4224 \%$ & 0,51703 & $3,4068 \%$ \\
\hline
\end{tabular}




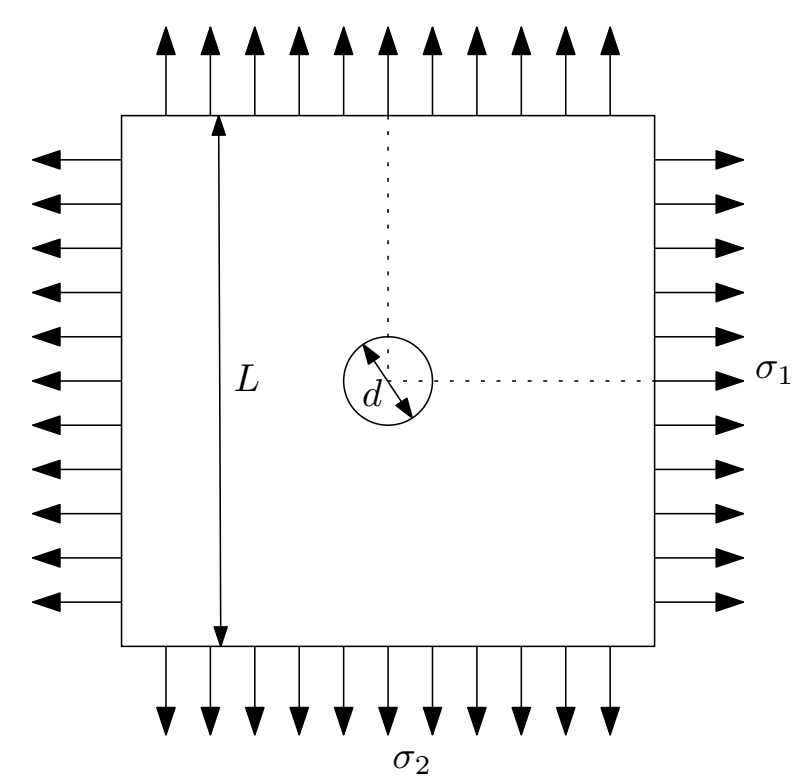

Figura 6.18: Problema da placa com furo

Tabela 6.7: Concentradores de tensão para uma placa com furo central

\begin{tabular}{|c|c|c|c|c|c|c|c|c|}
\hline \multicolumn{8}{|c|}{ Elementos quadráticos } \\
\hline \multicolumn{3}{|c|}{ Tração uniaxal } & \multicolumn{3}{c|}{ Cisalhamento puro } & \multicolumn{3}{c|}{ Tração biaxial } \\
\hline Nós & Fator & Erro & Nós & Fator & Erro & Nós & Fator & Erro \\
20 & 2,7666 & $-8,3918 \%$ & 20 & 3,6 & $-10,001 \%$ & 20 & 1,9332 & $-3,3408 \%$ \\
40 & 2,8846 & $-4,4846 \%$ & 40 & 3,8048 & $-4,8808 \%$ & 40 & 1,9644 & $-1,7821 \%$ \\
80 & 2,9912 & $-0,9529 \%$ & 80 & 3,9875 & $-0,31182 \%$ & 80 & 1,9949 & $-0,25413 \%$ \\
160 & 3,056 & $1,1934 \%$ & 160 & 4,0985 & $2,4614 \%$ & 160 & 2,0136 & $0,68137 \%$ \\
\hline \multicolumn{8}{|c|}{ Elementos isogeométricos - ordem 3 } \\
\hline \multicolumn{8}{|c|}{ Tração uniaxal Cisalhamento puro } & \multicolumn{3}{c|}{ Tração biaxial } \\
\hline Nós & Fator & Erro & Nós & Fator & Erro & Nós & Fator & Erro \\
65 & 2,60115 & $-13,8692 \%$ & 65 & 3,39461 & $-15,1348 \%$ & 65 & 1,88831 & $-5,58467 \%$ \\
115 & 2,97103 & $-1,62165 \%$ & 115 & 3,90651 & $-2,3372 \%$ & 115 & 2,03554 & $1,77702 \%$ \\
215 & 3,09338 & $2,42976 \%$ & 215 & 4,12366 & $3,0914 \%$ & 215 & 2,0631 & $3,15507 \%$ \\
\hline
\end{tabular}




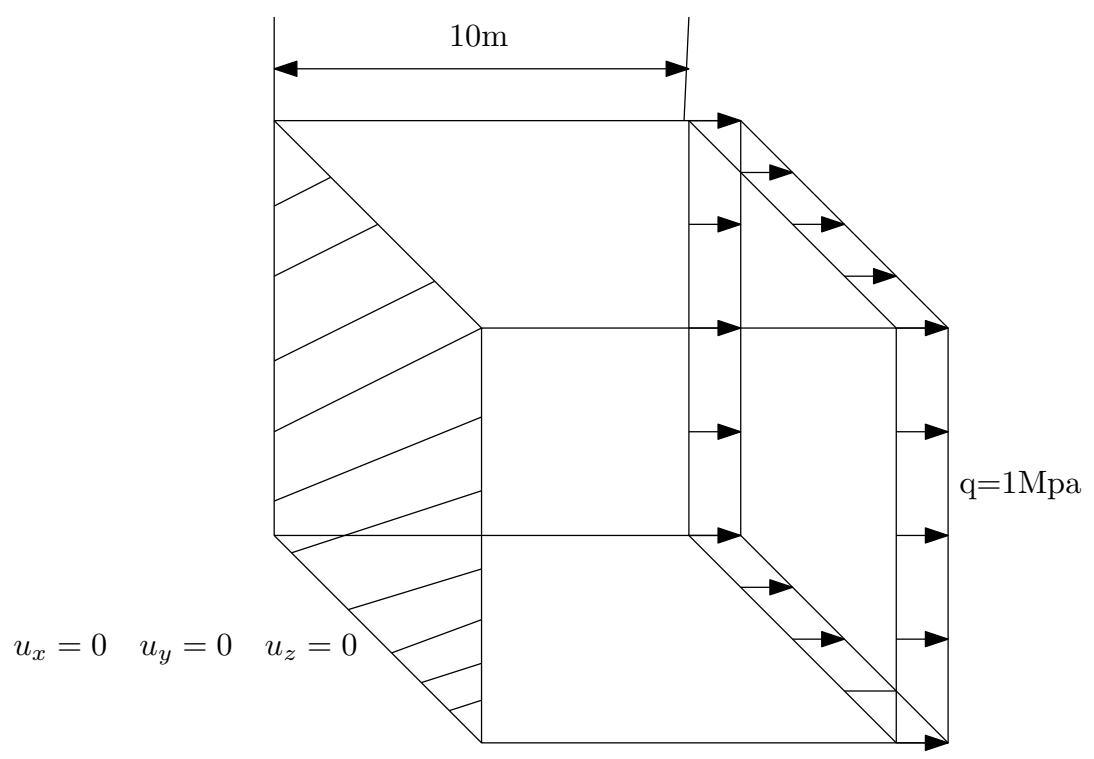

Figura 6.19: Cubo tracionado

obteve um erro RMS normalizado de 0,000031331 enquanto o isogeométrico com 24 pontos de controle obteve 0,000026275 .

Com esse exemplo, fica claro que para casos muito simples o uso do método isogeométrico converge para a solução analítica mais rapidamente, apresentando erro inferior a formulação constante mesmo com um número menor de graus de liberdade. No que diz respeito à economia de memória, esta é uma vantagem significativa. Entretanto, o método isogeométrico não apresenta vantagens significativas quando comparado ao padrão no que diz respeito ao tempo computacional, uma vez que calcular os elementos das matrizes de influência para o método de elementos de contorno padrão é significativamente mais rápido que o método dos elementos de contorno isogeométrico.

\subsubsection{Viga tridimensional}

Uma viga em balanço, de razão 10:1, que já foi analisada em um modelo bidimensional, é considerada para análise tridimensional como apresentado na figura 6.20. Na figura 6.21 observa-se a deflexão calculada para diferentes discretizações. Com 2088 pontos de controle, e curvas de ordem 2 , foi alcançado um erro relativo de 1,58\%.

\subsubsection{Cilindro de parede espessa}

Para analisar a precisão das medidas de tensão, um cilindro com uma pressão hidrostática interna de $50 \mathrm{~Pa}$ é considerado. Um módulo de elasticidade de $26 \mathrm{kPa}$ e um coeficiente de Poisson de 0,3 foram utilizados. Esse problema foi apresentado em Kane (1994) onde a solução analítica para as tensões radiais, $\sigma_{r}=50 \mathrm{~Pa}$, e circunferenciais, $\sigma_{\theta}=62,5 \mathrm{~Pa}$, foram mostradas. Na análise isogeométrica, como não existe aproximação 


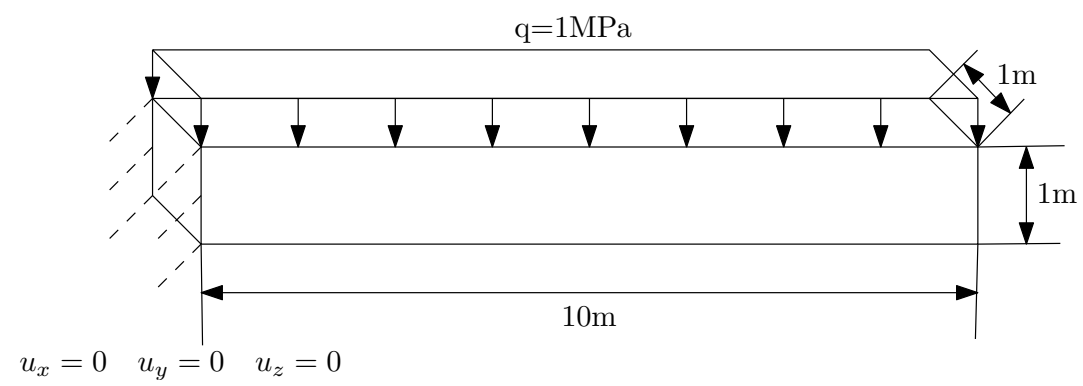

Figura 6.20: Viga tridimensional com carregamento distribuído

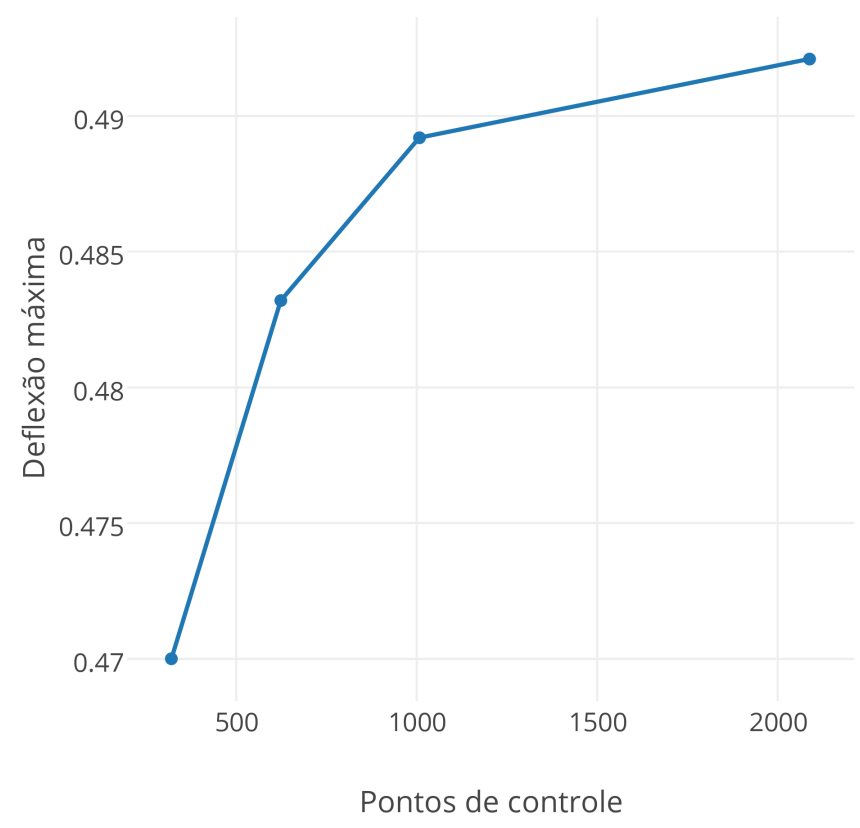

Figura 6.21: Deflexão máxima de uma viga de razão 10:1 


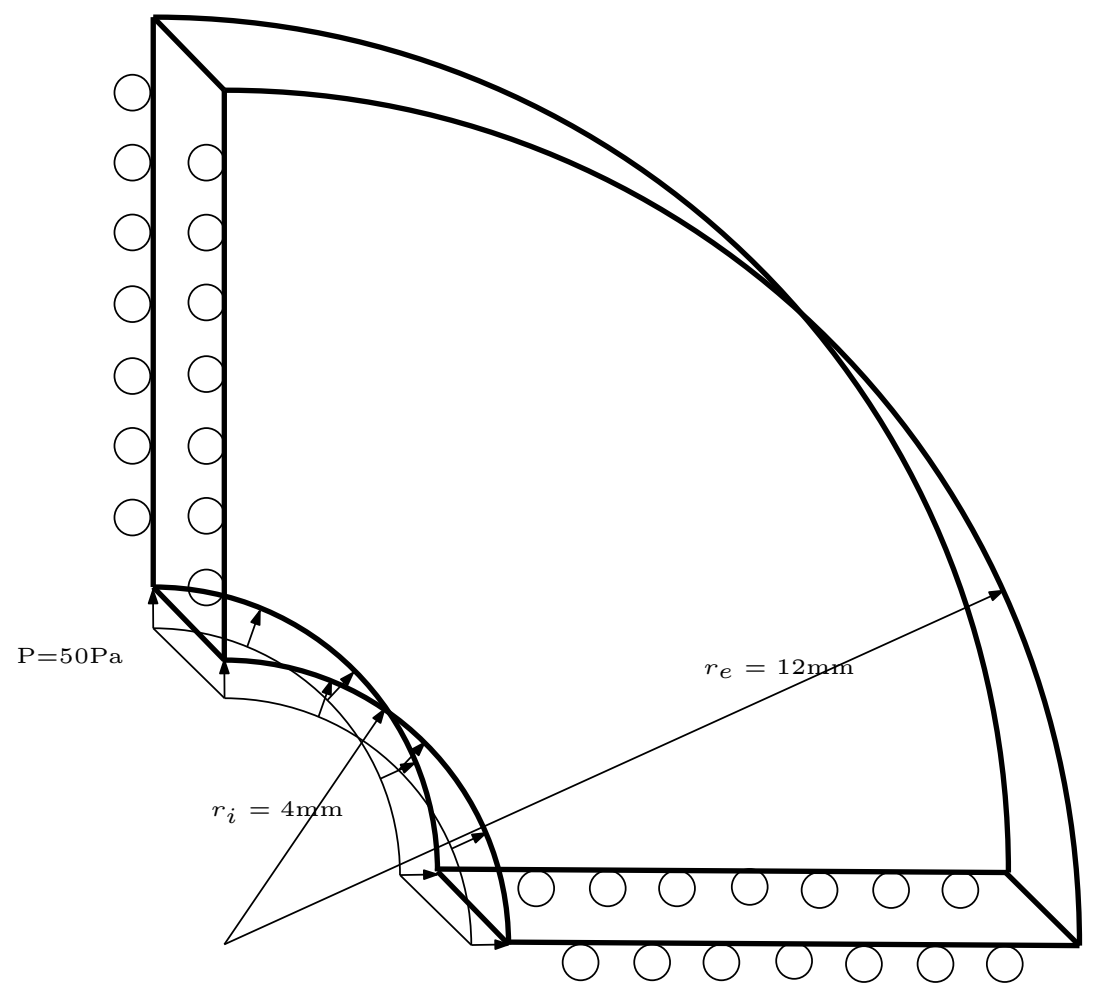

Figura 6.22: Cilindro de parede espessa com pressão hidrostática

Tabela 6.8: Tensão circunferencial para um cilindro de parede espessa

\begin{tabular}{|c|c|c|c|}
\hline \multicolumn{2}{|c|}{ Elemento quadrático padrão } & \multicolumn{2}{c|}{ KANE } \\
\hline Nós & Erro relativo $-\sigma_{\theta}$ & & \multicolumn{2}{c|}{ Elemento isogeométrico - ordem 3} \\
\hline 290 & $0,2512 \%$ & 54 & Erro relativo $-\sigma_{\theta}$ \\
& & 90 & $0,0272 \%$ \\
& & $5,0032 \%$ \\
\hline
\end{tabular}

nas normais, as tensões radiais são exatas, pois, nesse caso, dependem apenas das condições de contorno e das normais.

Na tabela 6.8 estão os erros máximos do método isogeométrico e do resultado apresentado por Kane (1994). Mesmo comparando com elementos de mesma ordem os resultados do método isogeométrico tiveram erros menores.

\subsection{Análise do uso da aproximação cruzada adaptativa}

\subsubsection{Placa quadrada com furos}

Para testar a eficiência da ACA em termos do tempo de execução do método, uma placa quadrada com uma quantidade crescente de furos regularmente distribuídos é analisada. A figura 6.23 representa o caso com 16 furos. O tamanho dos furos é modificado de modo que a razão entre as a área dos furos e a área da placa seja constante e igual a $12,47 \%$. 


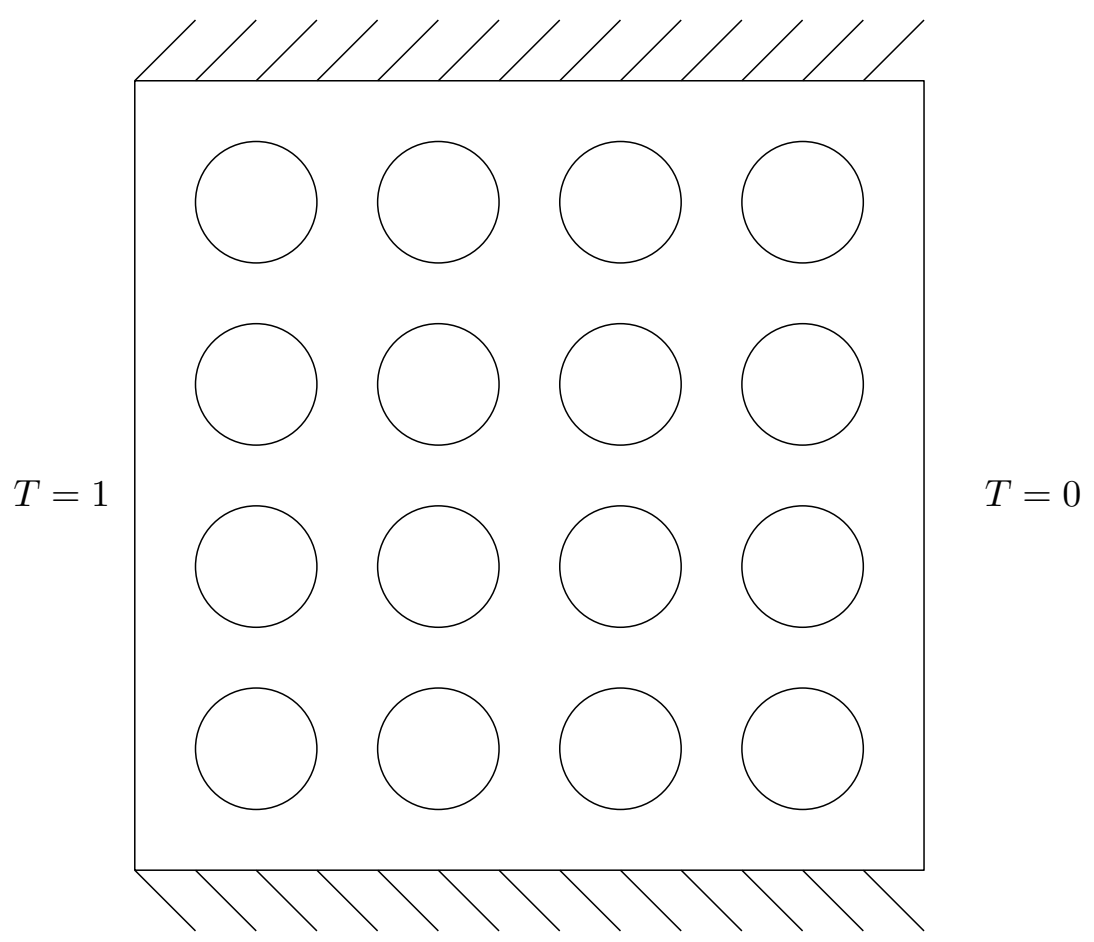

Figura 6.23: Placa com furos

Calculando o fluxo em um dos lados da placa e dividindo pelo fluxo da placa sem furos, pode-se calcular uma condutividade térmica equivalente que é mostrada na figura 6.24. Observa-se, então, que o valor da condutividade converge a medida que a quantidade de furos aumenta.

Na figura 6.25 está representada o tempo de execução variando a quantidade de furos, indo de apenas 1 até 121 furos. Pela inclinação das retas, pode-se verificar uma evidente mudança da complexidade do método, sendo o padrão próximo à $n^{2}$ e o acelerado próximo à $n \log n$. A diferença de inclinação faz com que o método acelerado, apesar de mais lento para problemas pequenos, fique mais rápido para problemas com mais de 500 graus de liberdade. O alto custo das funções de base maximiza os efeitos do pivotamento parcial do ACA fazendo com que o uso da ACA na formulação isogeométrica se mostrasse surpreendentemente vantajoso mesmo para problemas com baixo número de graus de liberdade.

Os parâmetros utilizados na ACA nesse problema foram, $\varepsilon=10^{-5}, \eta=0,5$ e 100 como número máximo de nós por folha.

A figura 6.26 relaciona o ganho em desempenho com os distintos valores de $\eta$ para o caso de 100 furos. Apesar de variar com a geometria, o valor ótimo de $\eta=0,5$ não se afasta significativamente disso e, por isso, foi utilizado em todos os casos desse trabalho. Ao diminuir o valor de $\eta$ a condição de admissibilidade fica mais rígida gerando mais blocos inadmissíveis. Como consequência, os blocos admissíveis tem um posto menor e podem ser aproximados de maneira mais eficaz. 


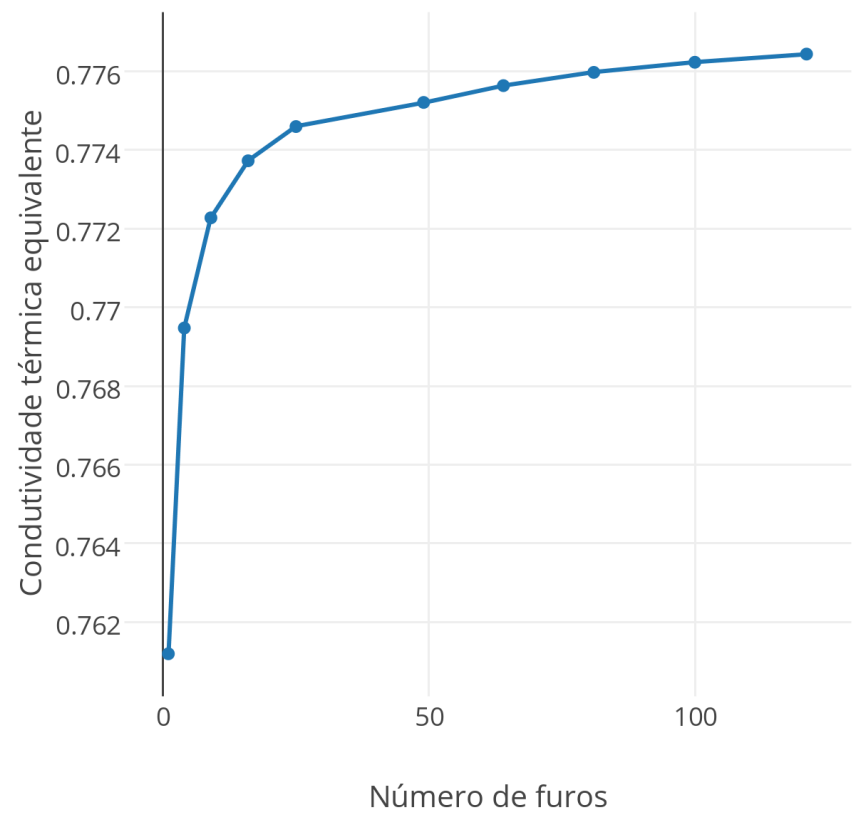

Figura 6.24: Condutividade térmica equivalente para quantidades distintas de furos.

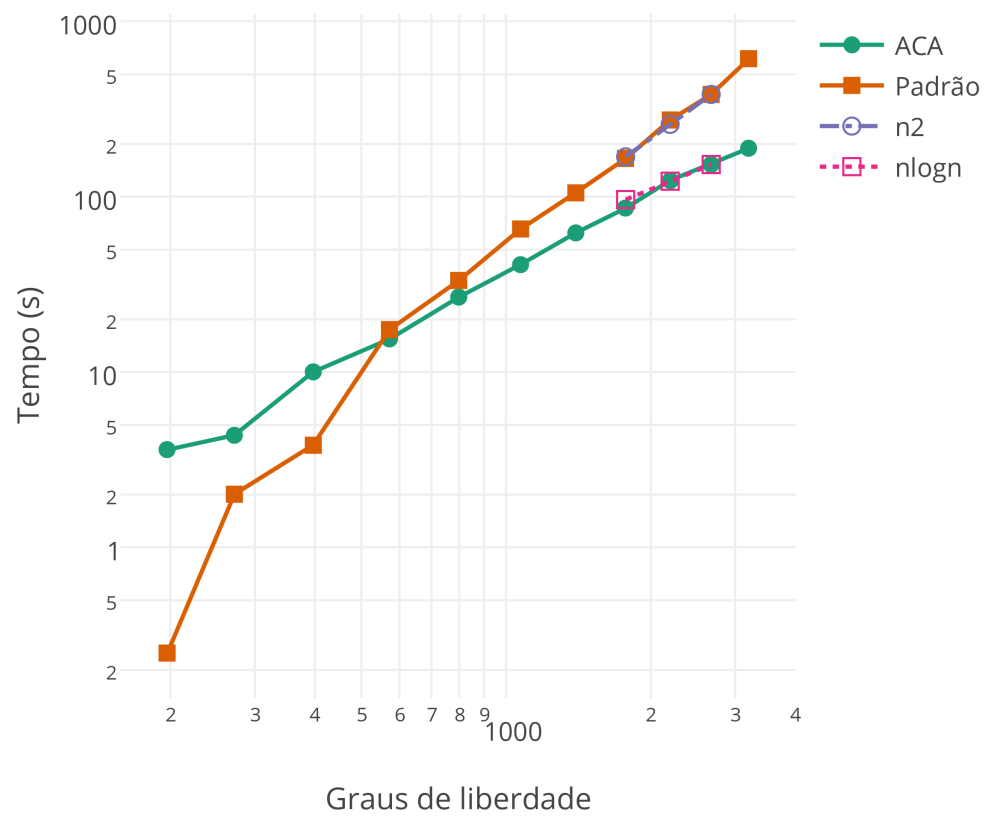

Figura 6.25: Tempo de execução do programa para a placa com distintas quantidades de furos. 


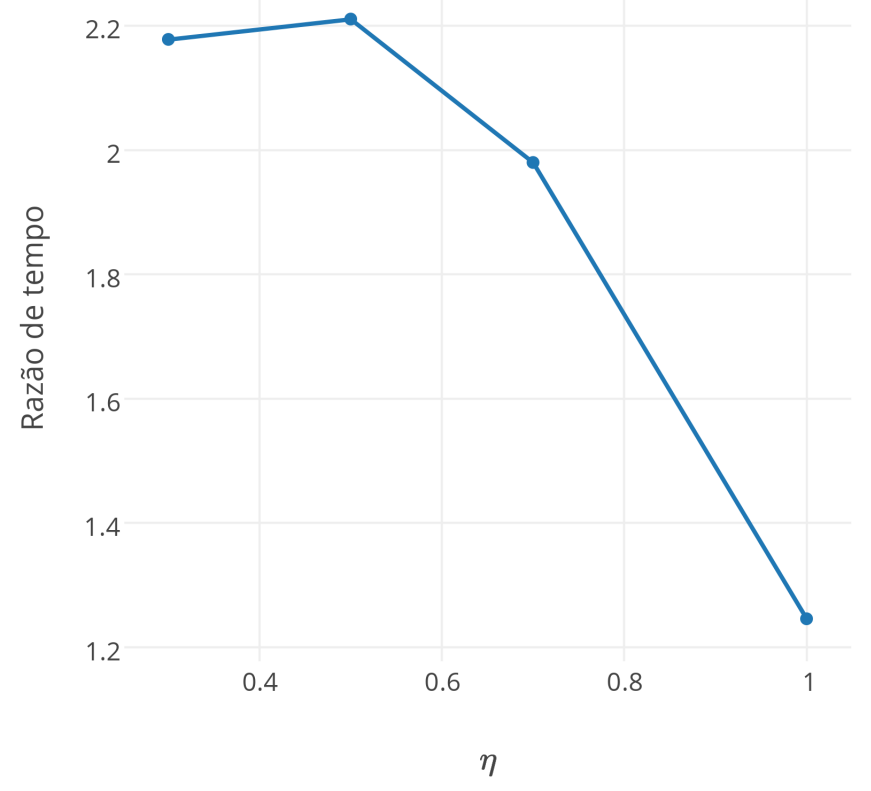

Figura 6.26: Tempo de execução do programa para a placa com distintos valores de $\eta$.

De acordo com Benedetti, Milazzo e Aliabadi (2011), o valor do erro do ACA deve ter ordem menor que a menor grandeza analisada. O valor de $\varepsilon=10^{-5}$ satisfaz essa condição nos casos estudados. O valor de cardinalidade igual a 100 é maior que do que o recomendado usualmente, mas como a montagem da matriz é o processo mais custoso desse método, um valor mais alto é mais interessante no método isogeométrico (BRANCATI, 2010).

\subsubsection{Cubo com furos}

Um cubo com 27 furos esféricos, conforme figura 6.27, sob tração será analisado nessa seção. Considerando $E=210 G P a$ e $\mu=0,28$ e um carregamento constante e igual a $1 \mathrm{~Pa}$ em uma das faces, é possível encontrar um módulo de elasticidade equivalente comparando o deslocamento do cubo com furos e o de um furo sem furos. $\mathrm{O}$ problema foi analisado com o método dos elementos de contorno padrão e com o método isogeométrico com uma superfície de ordem 3. A tabela 6.9 mostra o valor da razão entre o deslocamento com furos e o deslocamento sem furos para método isogoemétrico e o tempo gasto pelo método com e sem a utilização da ACA. Para o método padrão, com 35748 nós obtém-se uma razão entre deslocamentos de 0,8075. Percebe-se, então, que, com uma quantidade de nós muito inferior ao do método padrão, se obtém um 


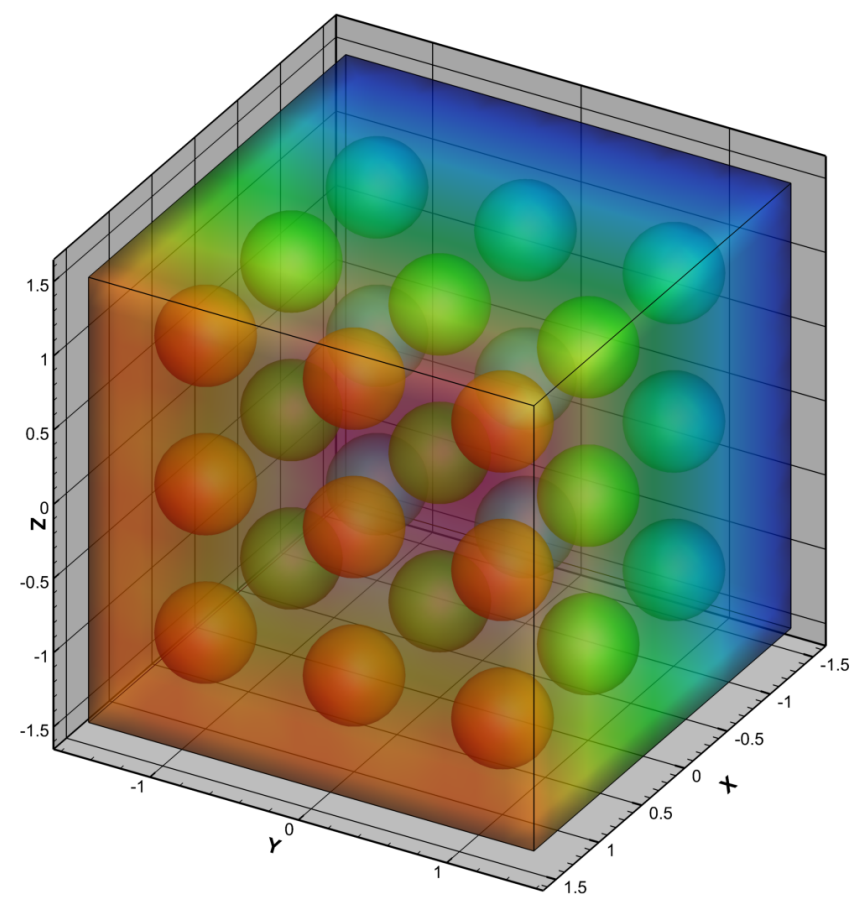

Figura 6.27: Cubo com 27 furos sob tração.

Tabela 6.9: Modulo de elasticidade equivalente para um cubo com 27 furos

\begin{tabular}{|c|c|c|c|}
\hline \multicolumn{4}{|c|}{ Elemento isogeométrico - ordem 3 } \\
\hline Nós & Razão entre deslocamentos & Tempo padrão & Tempo ACA \\
\hline 609 & 0,7732 & 54,59 & 60,32 \\
1023 & 0,7735 & 168,51 & 80,47 \\
\hline
\end{tabular}

resultado próximo entre os dois métodos. Além disso, no método isogeométrico, com 1023 nós o método com ACA foi duas vezes mais rápido que o padrão. 


\section{CONSIDERAÇÕES FINAIS}

\subsection{Conclusão}

Este trabalho apresentou uma formulação isogeométrica do método dos elementos de contorno acelerada pela aproximação adaptativa cruzada. Como as NURBS são indiscutivelmente a entidade mais usada nos programas CAD, elas foram escolhidas como as entidades do método isogeométrico. Com isso o método se tornou capaz de representar exatamente a geometria dos problemas analisados além de ter como base funções suaves de alta continuidade.

A definição de pontos fontes usando as coordenadas de greville modificada facilita em muito a formulação uma vez que os pontos não se posicionam mais nos cantos do problema, evitando ter de realizar um tratamento especial dos termos diagonais nesses casos. Vale ressaltar que a posição dos pontos de colocação, por estar próximo ao canto, demanda uma atenção especial nas integrais numéricas do método devido a quasi-singularidade forte naquela região. Esse efeito é ainda aumentado quando existe uma concentração dos pontos de controle, como no caso da esfera.

Foi apresentada uma técnica para aplicar as condições de contorno que leva em consideração as características da ACA no intuito de não perder as vantagens relativas ao custo de armazenamento e ao produto matriz-vetor. As grandezas obtidas na solução do sistema ficam nos pontos de controle e é necessária uma transformação para obter os resultados no contorno.

A geração de uma malha de qualidade para problemas tridimensionais ainda é um processo trabalhoso e complexo, apesar de mais simples do que nos métodos de domínio. Portanto, pode ser considerada uma das maiores vantagens do método apresentado não ser necessária a geração de malha uma vez que a discretização é obtida diretamente dos programas CAD.

Apesar das matrizes hierárquicas terem sido idealizadas para tratar problemas com muitos graus de liberdade, esse método se mostrou uma combinação interessante com o método isogeométrico. Devido ao alto custo das funções de base e a necessidade de uma grande quantidade de pontos de integração, as vantagens da ACA se apresentam muito antes do que o método convencional. Essa propriedade é importante porque o método é capaz de representar geometrias complexas com poucos pontos de controle e teve, também uma convergência mais rápida do que o método padrão. Além disso, a diferença entre as ordens do método, $n^{2}$ para o padrão e $n \log n$ para o acelerado, fará com que a diferença de velocidade do método aumente rapidamente com o aumento da quantidade de nós. 
Na análise bidimensional potencial, mostrou-se a importância de tratar de maneira diferenciada as condições de contorno do método isogeométrico. O método teve uma alta precisão com poucos graus de liberdade, tendo um desempenho ligeiramente melhor que o método padrão nos casos analisados. Nos problemas elásticos bidimensionais, o método proposto, com distintas ordens, teve um comportamento semelhante ao método padrão com elementos quadráticos contínuos enquanto o método com elementos constantes teve uma convergência muito lenta quando comparado aos demais.

Nos problemas potenciais tridimensionais, o método isogemétrico alcançou erros muito baixos com poucos pontos de controle. No caso do cubo, por exemplo, com uma quantidade de nós 200 vezes menor que o método padrão, o método teve um erro RMS menor que o padrão.

A combinação do método isogeométrico e o ACA se provou robusto e eficiente para a modelagem de problemas potenciais e elásticos, bidimensionais e tridimensionais, melhorando a acurácia e a velocidade quando comparado ao método padrão, além de eliminar as horas do engenheiro usadas com a geração e ajuste de malhas.

\subsection{Trabalhos futuros}

A extensão natural do trabalho é desenvolver uma metodologia para tratar superfícies aparadas, tornando o método capaz de lidar com qualquer geometria. As integrais numéricas quase singulares do método podem ser estudadas e melhoradas, principalmente, em três dimensões.

O método apresentado pode ser facilmente estendido para os mais diversos problemas, trocando apenas a formulação de elementos de contorno. Problemas onde uma representação geométrica de qualidade tenha grande influência como:

- Problemas de contato;

- Problemas de fronteira móvel;

- Otimização de forma;

são certamente os problemas onde o método isogeométrico apresentará as maiores vantagens quando comparado com o método dos elementos contorno padrão e também com outros métodos numéricos como, por exemplo, o método dos elementos finitos. 


\section{Referências Bibliográficas}

AOKI, S. et al. Fast multipole boundary element analysis of corrosion problems. CMES-COMPUTER MODELING IN ENGINEERING \& SCIENCES, v. 6, n. 2, p. 123-131, 2004.

AUTODESK, A. T. Splines Plug-in for Rhino user manual. [S.l.]: Autodesk, 2012.

BAKER, A. H.; JESSUP, E. R.; KOLEV, T. V. A simple strategy for varying the restart parameter in gmres $(\mathrm{m})$. Journal of computational and applied mathematics, Elsevier, v. 230, n. 2, p. 751-761, 2009.

BAKER, A. H.; JESSUP, E. R.; MANTEUFFEL, T. A technique for accelerating the convergence of restarted gmres. SIAM Journal on Matrix Analysis and Applications, SIAM, v. 26, n. 4, p. 962-984, 2005.

BANERJEE, P. K.; BUTTERFIELD, R. Boundary element methods in engineering science. [S.l.]: McGraw-Hill London, 1981. v. 17.

BARRA, L. et al. Iterative solution of bem equations by gmres algorithm. Computers 65 structures, Elsevier, v. 44, n. 6, p. 1249-1253, 1992.

BAZILEVS, Y.; AKKERMAN, I. Large eddy simulation of turbulent taylor-couette flow using isogeometric analysis and the residual-based variational multiscale method. Journal of Computational Physics, Elsevier, v. 229, n. 9, p. 3402-3414, 2010.

BAZILEVS, Y. et al. Isogeometric fluid-structure interaction: theory, algorithms, and computations. Computational mechanics, Springer, v. 43, n. 1, p. 3-37, 2008.

BAZILEVS, Y. et al. Isogeometric analysis using t-splines. Computer Methods in Applied Mechanics and Engineering, Elsevier, v. 199, n. 5, p. 229-263, 2010.

BAZILEVS, Y.; HSU, M.-C.; SCOTT, M. Isogeometric fluid-structure interaction analysis with emphasis on non-matching discretizations, and with application to wind turbines. Computer Methods in Applied Mechanics and Engineering, Elsevier, v. 249, p. 28-41, 2012.

BEBENDORF, M. Approximation of boundary element matrices. Numerishce Mathematik, v. 86, p. 565-589, 2000.

BEBENDORF, M. Hierarchical matrices. [S.l.]: Springer, 2008.

BEBENDORF, M.; GRZHIBOVSKIS, R. Accelerating galerkin bem for linear elasticity using adaptive cross approximation. Mathematical Methods in the Applied Sciences, v. 29, p. 1721-1747, 2006.

BEBENDORF, M.; RJASANOW, S. Adaptive low-rank approximation of collocation matrices. Computing, v. 70, p. 1-24, 2003.

BECKER, A. A. The boundary element method in engineering: a complete course. [S.l.]: McGraw-Hill Companies, 1992. 
BEER, G. Programming the boundary element method. [S.1.]: John Wiley \& Sons, Inc., 2000.

BEER, G. Advanced Numerical Simulation Methods: From CAD Data Directly to Simulation Results. [S.1.]: CRC Press, 2015.

BEER, G.; MARUSSIG, B.; ZECHNER, J. A simple approach to the numerical simulation with trimmed cad surfaces. Computer Methods in Applied Mechanics and Engineering, Elsevier, v. 285, p. 776-790, 2015.

BELYTSCHKO, T. et al. Element free galerkin methods. International journal for numerical methods in engineering, John Wiley \& Sons, Ltd, v. 37, n. 2, p. 229-256, 1994.

BENEDETTI, I.; MILAZZO, A.; ALIABADI, M. Boundary element methods in engineering and sciences, vol 4. In: [S.l.]: Imperial College Press, 2011. cap. Fast hierarchical boundary element method for large-scale 3-D elastic problems.

BENSON, D. et al. Blended isogeometric shells. Computer Methods in Applied Mechanics and Engineering, Elsevier, v. 255, p. 133-146, 2013.

BERDINSKY, D. et al. Dimensions and bases of hierarchical tensor-product splines. Journal of Computational and Applied Mathematics, Elsevier, v. 257, p. 86-104, 2014.

BÖRM, S.; GRASEDYCK, L. Hybrid cross approximation of integral operators. Numerische Mathematik, Springer, v. 101, n. 2, p. 221-249, 2005.

BORNEMANN, P.; CIRAK, F. A subdivision-based implementation of the hierarchical b-spline finite element method. Computer Methods in Applied Mechanics and Engineering, Elsevier, v. 253, p. 584-598, 2013.

BRACCO, C. et al. Trigonometric generalized t-splines. Computer Methods in Applied Mechanics and Engineering, Elsevier, v. 268, p. 540-556, 2014.

BRAGA, L. M. O método dos elementos de contorno rápido com expansão em multipólos aplicado a problemas de condução de calor. 2013.

BRANCATI, A. Boundary element method for fast solution of acoustic problems: active and passive noise control. Tese (Doutorado) - Imperial College London, 2010.

BRANCATI, A.; ALIABADI, M. H.; BENEDETTI, I. Hierarchical adaptive cross approximation gmres technique for solution of acoustic problems using the boundary element method. CMES Computer Modeling in Engineering and Sciences, v. 45, p. 149-172, 2009.

BREBBIA, C. A. Boundary element methods in engineering. [S.1.]: Springer New York, NY, USA:, 1982.

BREBBIA, C. A.; DOMINGUEZ, J. Boundary elements: an introductory course. [S.l.]: WIT press, 1996.

BREBBIA, C. A.; WALKER, S. Boundary element techniques in engineering. [S.1.]: Elsevier, 2013. 
BREBBIA, C. A.; WROBEL, L. C.; TELLES, J. C. F. Boundary Element Techniques: Theory and Applications in Engineering. [S.l.]: WIT Press, 1984.

BRESSAN, A. Some properties of lr-splines. Computer Aided Geometric Design, Elsevier, v. 30, n. 8, p. 778-794, 2013.

BUCHER, H. F. et al. A novel approach to applying fast wavelet transforms in the boundary element method. Electronic Journal of Boundary Elements, v. 2, p. 187-195, 2002 .

BUFFA, A.; CHO, D.; SANGALLI, G. Linear independence of the t-spline blending functions associated with some particular t-meshes. Computer Methods in Applied Mechanics and Engineering, Elsevier, v. 199, n. 23, p. 1437-1445, 2010.

BUFFA, A.; SANGALLI, G.; VÁZQUEZ, R. Isogeometric methods for computational electromagnetics: B-spline and t-spline discretizations. Journal of Computational Physics, Elsevier, v. 257, p. 1291-1320, 2014.

CABRAL et al. A bem formulation using b-splines: I-uniform blending functions. Engineering Analysis with Boundary Elements, Elsevier, v. 7, n. 3, p. 136-144, 1990.

CABRAL; WROBEL, L.; BREBBIA, C. A bem formulation using b-splines: Ii-multiple knots and non-uniform blending functions. Engineering Analysis with Boundary Elements, v. 8, p. 51-55, 1991.

CÁRDENAS, S.; RUBÉN, o. Nurbs-enhanced finite element method (nefem). Universitat Politècnica de Catalunya, 2009.

CARRIER, J.; GREENGARD, L.; ROKHLIN, V. A fast adaptive multipole algorithm for particle simulations. SIAM journal on scientific and statistical computing, SIAM, v. 9 , n. 4 , p. 669-686, 1988.

CHENG, H.; GREENGARD, L.; ROKHLIN, V. A fast adaptive multipole algorithm in three dimensions. Journal of computational physics, Elsevier, v. 155, n. 2, p. 468-498, 1999.

CHEW, W. et al. Review of large scale computing in electromagnetics with fast integral equation solvers. Computer Modeling in Engineering and Sciences, v. 5, n. 4, p. 361-372, 2004.

COLLIER, N. et al. The cost of continuity: a study of the performance of isogeometric finite elements using direct solvers. Computer Methods in Applied Mechanics and Engineering, Elsevier, v. 213, p. 353-361, 2012.

COSTA, D. I. G. Nova formulação do método dos elementos de contorno para associação de placas espessas. 2015.

COSTANTINI, P. et al. Quasi-interpolation in isogeometric analysis based on generalized b-splines. Computer Aided Geometric Design, Elsevier, v. 27, n. 8, p. 656-668, 2010.

COTTRELL, J. et al. Isogeometric analysis of structural vibrations. Computer methods in applied mechanics and engineering, Elsevier, v. 195, n. 41, p. 5257-5296, 2006. 
COTTRELL, J. A.; HUGHES, T. J. R.; BAZILEVS, Y. Isogeometric analysis toward integration of CAD and FEA. [S.l.]: Wiley, 2009.

CROTTY, J. A block equation solver for large unsymmetric matrices arising in the boundary integral equation method. International Journal for Numerical Methods in Engineering, Wiley Online Library, v. 18, n. 7, p. 997-1017, 1982.

CRUSE, T. Numerical solutions in three dimensional elastostatics. International journal of solids and structures, Elsevier, v. 5, n. 12, p. 1259-1274, 1969.

CRUSE, T.; RICHARDSON, J. Non-singular somigliana stress identities in elasticity. International Journal for Numerical Methods in Engineering, Wiley Online Library, v. 39, n. 19, p. 3273-3304, 1996.

DENG, J. et al. Polynomial splines over hierarchical t-meshes. Graphical models, Elsevier, v. 70, n. 4, p. 76-86, 2008.

DENG, X. et al. Isogeometric analysis of continuum damage in rotation-free composite shells. Computer Methods in Applied Mechanics and Engineering, North-Holland, v. 284, p. 349-372, 2015.

DIMITRI, R. et al. Isogeometric large deformation frictionless contact using t-splines. Computer methods in applied mechanics and engineering, Elsevier, v. 269, p. 394-414, 2014.

DOKKEN, T.; LYCHE, T.; PETTERSEN, K. F. Polynomial splines over locally refined box-partitions. Computer Aided Geometric Design, Elsevier, v. 30, n. 3, p. 331-356, 2013.

DÖRFEL, M. R.; JÜTTLER, B.; SIMEON, B. Adaptive isogeometric analysis by local h-refinement with t-splines. Computer methods in applied mechanics and engineering, North-Holland, v. 199, n. 5, p. 264-275, 2010.

EIERMANN, M.; ERNST, O.; SCHNEIDER, O. Analysis of acceleration strategies for restarted minimal residual methods. Journal of Computational and Applied Mathematics, Elsevier, v. 123, n. 1, p. 261-292, 2000.

EMBREE, M. The tortoise and the hare restart gmres. SIAM review, SIAM, v. 45, n. 2, p. 259-266, 2003.

EVANS, E. et al. Hierarchical t-splines: Analysis-suitability, bézier extraction, and application as an adaptive basis for isogeometric analysis. Computer Methods in Applied Mechanics and Engineering, Elsevier, v. 284, p. 1-20, 2015.

FARIN, G. E. Curves and Surfaces for Computer-Aided Geometric Design: A Practical Code. [S.l.]: Academic Press, Inc., 1996.

FISCHER, P. et al. Isogeometric analysis of $2 \mathrm{~d}$ gradient elasticity. Computational Mechanics, Springer, v. 47, n. 3, p. 325-334, 2011.

FREDHOLM, I. Sur une classe d'équations fonctionnelles. Acta mathematica, Springer, v. 27, n. 1, p. 365-390, 1903. 
GIANNELLI, C.; JÜTTLER, B.; SPELEERS, H. Strongly stable bases for adaptively refined multilevel spline spaces. Advances in Computational Mathematics, Springer, v. 40, n. 2, p. 459-490, 2014.

GINNIS, A. et al. Isogeometric boundary-element analysis for the wave-resistance problem using t-splines. Computer Methods in Applied Mechanics and Engineering, Elsevier, v. 279, p. 425-439, 2014.

GÓMEZ, H. et al. Isogeometric analysis of the cahn-hilliard phase-field model. Computer Methods in Applied Mechanics and Engineering, Elsevier, v. 197, n. 49, p. 4333-4352, 2008.

GOREINOV, S. A.; TYRTYSHNIKOV, E. E.; ZAMARASHKIN, N. L. A theory of pseudoskeleton approximations. Linear Algebra and its Applications, Elsevier, v. 261, n. 1, p. 1-21, 1997.

GRASEDYCK, L. Adaptive recompression of-matrices for bem. Computing, Springer, v. 74, n. 3, p. 205-223, 2005.

GRASEDYCK, L.; HACKBUSCH, W. Construction and arithmetics of h-matrices. Computing, Springer, v. 70, n. 4, p. 295-334, 2003.

GREENBAUM, A. Iterative methods for solving linear systems. [S.l.]: Siam, 1997. v. 17.

GREENGARD, L.; ROKHLIN, V. A fast algorithm for particle simulations. Journal of computational physics, Elsevier, v. 73, n. 2, p. 325-348, 1987.

GUIGGIANI, M. Formulation and numerical treatment of boundary integral equations with hypersingular kernels. Singular integrals in boundary element methods, Advances in Boundary Elements Series. Computational Mechanics Publications: Southampton, Boston, p. 85-124, 1998.

GUIGGIANI, M.; CASALINI, P. Direct computation of cauchy principal value integrals in advanced boundary elements. International Journal for Numerical Methods in Engineering, John Wiley \& Sons, Ltd, v. 24, n. 9, p. 1711-1720, 1987.

GUIGGIANI, M. et al. A general algorithm for the numerical solution of hypersingular boundary integral equations. Journal of applied mechanics, American Society of Mechanical Engineers, v. 59, n. 3, p. 604-614, 1992.

HABU, M.; NODERA, T. Gmres (m) algorithm with changing the restart cycle adaptively. In: CITESEER. Proceedings of Algorithmy 2000 Conference on Scientific Computing. [S.l.], 2000. p. 254-263.

HACKBUSCH, W. A sparse matrix arithmetic based on h-matrices. part i. International Journal for Numerical Methods in Engineering, v. 62, p. 89-108, 1999.

HACKBUSCH, W. A sparse matrix arithmetic based on h-matrices. part ii. International Journal for Numerical Methods in Engineering, v. 64, p. 21-47, 2000.

HACKBUSCH, W. Hierarchical Matrices: Algorithms and Analysis. [S.l.]: Springer, 2016. 
HACKBUSCH, W.; NOWAK, Z. P. On the fast matrix multiplication in the boundary element method by panel clustering. Numerische Mathematik, Springer, v. 54, n. 4, p. 463-491, 1989.

HAYAMI, K.; MATSUMOTO, H. Improvement of quadrature for nearly singular integrals in 3d-bem. WIT Transactions on Modelling and Simulation, WIT Press, v. 7,1994 .

HE, X.; LIM, K.-M.; LIM, S.-P. Fast bem solvers for 3d poisson-type equations. CMES: Computer Modeling in Engineering \& Sciences, v. 35, n. 1, p. 21-48, 2008.

HOSSEINI, S. et al. An isogeometric continuum shell element for non-linear analysis. Computer Methods in Applied Mechanics and Engineering, Elsevier, v. 271, p. 1-22, 2014 .

HUGHES, T. J.; REALI, A.; SANGALLI, G. Duality and unified analysis of discrete approximations in structural dynamics and wave propagation: comparison of p-method finite elements with k-method nurbs. Computer methods in applied mechanics and engineering, Elsevier, v. 197, n. 49, p. 4104-4124, 2008.

HUGHES, T. J. R.; COTTRELL, J. A.; BAZILEVS, Y. Isogeometric analysis: Cad, finite elements, nurbs, exact geometry and mesh refinement. Comput. Methods Appl. Mech. Engrg, v. 194, p. 4135-4195, 2005.

JASWON, M. Integral equation methods in potential theory. i. In: THE ROYAL SOCIETY. Proceedings of the Royal Society of London A: Mathematical, Physical and Engineering Sciences. [S.1.], 1963. v. 275, n. 1360, p. 23-32.

JOHANNESSEN, K.; ANDRÉ, K.; DOKKEN, T. T. Isogeometric analysis using lr b-splines. Computer Methods in Applied Mechanics and Engineering, Elsevier, v. 269, p. $471-514,2014$.

JOUBERT, W. On the convergence behavior of the restarted gmres algorithm for solving nonsymmetric linear systems. Numerical linear algebra with applications, Wiley Online Library, v. 1, n. 5, p. 427-447, 1994.

KAGAN et al. Integrated mechanically based cae system using b-spline finite elements. Computer-Aided Design, Elsevier, v. 32, n. 8, p. 539-552, 2000.

KANE, J.; KUMAR, B.; KASHAVA, S. S. An arbitrary condensing, noncondensing solution strategy for large scale, multi-zone boundary element analysis. Computer Methods in Applied Mechanics and Engineering, Elsevier, v. 79, n. 2, p. 219-244, 1990.

KANE, J. H. Boundary element analysis in engineering continuum mechanics. Englewood Cliffs, NJ: Prentice Hall, 1994., 1994.

KANG, H.; CHEN, F.; DENG, J. Modified t-splines. Computer Aided Geometric Design, Elsevier, v. 30, n. 9, p. 827-843, 2013.

KATSIKADELIS, J. The Boundary Element Method for Engineers and Scientists: Theory and Applications. [S.l.]: Academic Press, 2016. 
KATSIKADELIS, J. T. Boundary Elements: Theory and Applications. [S.1.]: Elsevier, 2002.

KIENDL, J. et al. The bending strip method for isogeometric analysis of kirchhofflove shell structures comprised of multiple patches. Computer Methods in Applied Mechanics and Engineering, Elsevier, v. 199, n. 37, p. 2403-2416, 2010.

KIENDL, J.; BLETZINGER, K.; LINHARD J. WÜCHNER, R. Isogeometric shell analysis with kirchhoff-love elements. Computer Methods in Applied Mechanics and Engineering, Elsevier, v. 198, n. 49, p. 3902-3914, 2009.

KIM, H.-J.; SEO, Y.-D.; YOUN, S.-K. Isogeometric analysis with trimming technique for problems of arbitrary complex topology. Computer Methods in Applied Mechanics and Engineering, Elsevier, v. 199, n. 45, p. 2796-2812, 2010.

KIM, H.-J.; YOUN, S.-K. Spline-based meshfree method. International Journal for Numerical Methods in Engineering, Wiley Online Library, v. 92, n. 9, p. 802-834, 2012.

KISS, G.; GIANNELLI, C.; JÜTTLER, B. Algorithms and data structures for truncated hierarchical b-splines. In: Mathematical Methods for Curves and Surfaces. [S.1.]: Springer, 2012. p. 304-323.

KURU, G. et al. Goal-adaptive isogeometric analysis with hierarchical splines. Computer Methods in Applied Mechanics and Engineering, Elsevier, v. 270, p. 270-292, 2014.

KURZ, S.; RAIN, O.; RJASANOW, S. Fast boundary element methods in computational electromagnetism. In: Boundary Element Analysis. [S.l.]: Springer, 2007. p. 249-279.

KUTT, H. The numerical evaluation of principal value integrals by finite-part integration. Numerische Mathematik, Springer, v. 24, n. 3, p. 205-210, 1975.

LACHAT, J.; WATSON, J. Effective numerical treatment of boundary integral equations: a formulation for three-dimensional elastostatics. International Journal for Numerical Methods in Engineering, Wiley Online Library, v. 10, n. 5, p. 991-1005, 1976.

LEUNG, C.; WALKER, S. Iterative solution of large three-dimensional bem elastostatic analyses using the gmres technique. International Journal for numerical methods in engineering, Wiley Online Library, v. 40, n. 12, p. 2227-2236, 1997.

LI, B. et al. Surface mesh to volumetric spline conversion with generalized polycubes. Visualization and Computer Graphics, IEEE Transactions on, IEEE, v. 19, n. 9, p. 1539-1551, 2013.

LI, K.; QIAN, X. Isogeometric analysis and shape optimization via boundary integral. Computer-Aided Design, Elsevier, v. 43, n. 11, p. 1427-1437, 2011.

LI, X.; DENG, J.; CHEN, F. Surface modeling with polynomial splines over hierarchical t-meshes. The Visual Computer, Springer, v. 23, n. 12, p. 1027-1033, 2007. 
LI, X.; DENG, J.; CHEN, F. Polynomial splines over general t-meshes. The Visual Computer, Springer, v. 26, n. 4, p. 277-286, 2010.

LI, X.; SCOTT, M. A. Analysis-suitable t-splines: Characterization, refineability, and approximation. Mathematical Models and Methods in Applied Sciences, v. 24, n. 06, p. 1141-1164, 2014.

LIU, Y. Fast multipole boundary element method: theory and applications in engineering. [S.1.]: Cambridge university press, 2009.

LU, J. Circular element: Isogeometric elements of smooth boundary. Computer methods in applied mechanics and engineering, Elsevier, v. 198, n. 30, p. 2391-2402, 2009 .

LU, J. Isogeometric contact analysis: Geometric basis and formulation for frictionless contact. Computer Methods in Applied Mechanics and Engineering, Elsevier, v. 200, n. 5, p. $726-741,2011$.

LU, J.; ZHOU; XIANLIAN. Cylindrical element: Isogeometric model of continuum rod. Computer Methods in Applied Mechanics and Engineering, Elsevier, v. 200, n. 1, p. 233-241, 2011.

MANNI, C.; PELOSI, F.; SAMPOLI, M. L. Generalized b-splines as a tool in isogeometric analysis. Computer Methods in Applied Mechanics and Engineering, Elsevier, v. 200, n. 5, p. 867-881, 2011.

MANSUR, W.; ARAUJO, F.; MALAGHINI, J. Solution of bem systems of equations via iterative techniques. International Journal for Numerical Methods in Engineering, Wiley Online Library, v. 33, n. 9, p. 1823-1841, 1992.

MARCZAK, R. J.; CREUS, G. J. Direct evaluation of singular integrals in boundary element analysis of thick plates. Engineering analysis with boundary elements, Elsevier, v. 26, n. 8, p. 653-665, 2002.

MARUSSIG, B. et al. Stable isogeometric analysis of trimmed geometries. arXiv preprint arXiv:1603.09660, 2016.

MASUD, A.; KANNAN, R. B-splines and nurbs based finite element methods for kohn-sham equations. Computer Methods in Applied Mechanics and Engineering, Elsevier, v. 241, p. 112-127, 2012.

MERKEL, M. et al. Iterative solution of large-scale 3d-bem industrial problems. Engineering Analysis with Boundary Elements, Elsevier, v. 22, n. 3, p. 183-197, 1998.

MIKHLIN, S. G. et al. Integral equations. [S.l.]: Pergamon press, 1964.

MORGENSTERN, P.; PETERSEIM, D. Adaptive analysis-suitable t-mesh refinement with linear complexity. arXiv preprint arXiv:140\%.6175, 2014.

NAGY, A. P.; BENSON, D. J. On the numerical integration of trimmed isogeometric elements. Computer Methods in Applied Mechanics and Engineering, Elsevier, v. 284, p. 165-185, 2015. 
NEUBER, H. Ni muskhelishvili, some basic problems of the mathematical theory of elasticity. 3. verb. und vermehrte auflage.(aus dem russischen übersetzt von jrm radok.) xxxi +704 s. m. 66 abb. groonigen (holl.) 1953. verlag p. noordhoff ltd. preis geb. 38.-fl. ZAMM-Journal of Applied Mathematics and Mechanics/Zeitschrift für Angewandte Mathematik und Mechanik, Wiley Online Library, v. 34, n. 12, p. 479-479, 1954.

NIELSEN, P. N. et al. Discretizations in isogeometric analysis of navier-stokes flow. Computer methods in applied mechanics and engineering, Elsevier, v. 200, n. 45, p. 3242-3253, 2011.

PEIGL, L.; TILLER, W. The NURBS book. [S.l.]: Berlin, Springer-Verlag,, 1996.

PILKEY, W. D. Formulas for stress, strain, and structural matrices. [S.l.]: John Wiley \& Sons, Inc., 1993.

RICHARDSON, J.; CRUSE, T. Weakly singular stress-bem for 2d elastostatics. International Journal for Numerical Methods in Engineering, Wiley Online Library, v. 45, n. 1, p. 13-35, 1999.

RIGBY, R.; ALIABADI, M. Out-of-core solver for large, multi-zone boundary element matrices. International journal for numerical methods in engineering, Wiley Online Library, v. 38, n. 9, p. 1507-1533, 1995.

RIZZO, F. J. An integral equation approach to boundary value problems of classical elastostatics. Quart. Appl. Math, v. 25, n. 1, p. 83-95, 1967.

ROGERS, D. F. An introduction to NURBS: with historical perspective. [S.1.]: Elsevier, 2000.

ROKHLIN, V. Rapid solution of integral equations of classical potential theory. Journal of Computational Physics, Elsevier, v. 60, n. 2, p. 187-207, 1985.

SAAD, Y.; SCHULTZ, M. H. Gmres: A generalized minimal residual algorithm for solving nonsymmetric linear systems. SIAM Journal on scientific and statistical computing, SIAM, v. 7, n. 3, p. 856-869, 1986.

SCHILLINGER, D. et al. An isogeometric design-through-analysis methodology based on adaptive hierarchical refinement of nurbs, immersed boundary methods, and t-spline cad surfaces. Computer Methods in Applied Mechanics and Engineering, Elsevier, v. 249, p. 116-150, 2012.

SCHILLINGER, D. et al. Isogeometric collocation: Cost comparison with galerkin methods and extension to adaptive hierarchical nurbs discretizations. Computer Methods in Applied Mechanics and Engineering, Elsevier, v. 267, p. 170-232, 2013.

SCHILLINGER, D.; RANK, E. An unfitted hp-adaptive finite element method based on hierarchical b-splines for interface problems of complex geometry. Computer Methods in Applied Mechanics and Engineering, Elsevier, v. 200, n. 47, p. 3358-3380, 2011. 
SCHMIDT, R.; WÜCHNER, R.; BLETZINGER, K.-U. Isogeometric analysis of trimmed nurbs geometries. Computer Methods in Applied Mechanics and Engineering, Elsevier, v. 241, p. 93-111, 2012.

SCOTT et al. Isogeometric boundary element analysis using unstructured t-splines. Computer Methods in Applied Mechanics and Engineering, Elsevier, v. 254, p. 197-221, 2013.

SCOTT, M. et al. Local refinement of analysis-suitable t-splines. Computer Methods in Applied Mechanics and Engineering, Elsevier, v. 213, p. 206-222, 2012.

SCOTT, M. A. et al. Isogeometric finite element data structures based on bézier extraction of t-splines. International Journal for Numerical Methods in Engineering, Wiley Online Library, v. 88, n. 2, p. 126-156, 2011.

SCOTT, M. A.; THOMAS, D. C.; EVANS, E. J. Isogeometric spline forests. Computer Methods in Applied Mechanics and Engineering, Elsevier, v. 269, p. 222-264, 2014.

SEDERBERG, T. W. et al. T-splines and t-nurccs. In: ACM. ACM transactions on graphics (TOG). [S.l.], 2003. v. 22, n. 3, p. 477-484.

SEDERBERG, T. W. et al. T-spline simplification and local refinement. In: ACM. Acm transactions on graphics (tog). [S.1.], 2004. v. 23, n. 3, p. 276-283.

SEVILLA, R.; FERNÁNDEZ-MÉNDEZ, S.; HUERTA, A. 3d nurbs-enhanced finite element method (nefem). International Journal for Numerical Methods in Engineering, Wiley Online Library, v. 88, n. 2, p. 103-125, 2011.

SHAW, A.; ROY, D. Nurbs-based parametric mesh-free methods. Computer methods in applied mechanics and engineering, Elsevier, v. 197, n. 17, p. 1541-1567, 2008.

SIMPSON et al. An isogeometric boundary element method for elastostatic analysis: 2d implementation aspects. Computers 86 Structures, Elsevier, v. 118, p. 2-12, 2013.

SIMPSON, R. N. et al. A two-dimensional isogeometric boundary element method for elastostatic analysis. Computer Methods in Applied Mechanics and Engineering, Elsevier, v. 209, p. 87-100, 2012.

SIMPSON, R. N. et al. Acoustic isogeometric boundary element analysis. Computer Methods in Applied Mechanics and Engineering, Elsevier, v. 269, p. 265-290, 2014.

SOMIGLIANA, C. Sopra l?equilibrio di un corpo elastico isotropo. [S.1.]: il Nuovo Ciemento., 1885. 17-20 p.

SOSONKINA, M. et al. A new adaptive gmres algorithm for achieving high accuracy. Numerical Linear Algebra with Applications, Wiley Online Library, v. 5, n. 4, p. 275-297, 1998.

SPELEERS, H. et al. Isogeometric analysis with powell-sabin splines for advection-diffusion-reaction problems. Computer Methods in Applied Mechanics and Engineering, Elsevier, v. 221, p. 132-148, 2012. 
SYMM, G. Integral equation methods in potential theory. ii. In: THE ROYAL SOCIETY. Proceedings of the Royal Society of London A: Mathematical, Physical and Engineering Sciences. [S.l.], 1963. v. 275, n. 1360, p. 33-46.

TELLES, J. C. F. A self-adaptive co-ordinate transformation for efficient numerical evaluation of general boundary element integrals. International Journal for Numerical Methods in Engineering, Wiley Online Library, v. 24, n. 5, p. 959-973, 1987.

TEMIZER, I.; WRIGGER; HUGHES, T. Three-dimensional mortar-based frictional contact treatment in isogeometric analysis with nurbs. Computer Methods in Applied Mechanics and Engineering, Elsevier, v. 209, p. 115-128, 2012.

TEMIZER, I.; WRIGGERS; HUGHES, T. Contact treatment in isogeometric analysis with nurbs. Computer Methods in Applied Mechanics and Engineering, Elsevier, v. 200, n. 9, p. 1100-1112, 2011.

THAI, C. H. et al. Static, free vibration, and buckling analysis of laminated composite reissner-mindlin plates using nurbs-based isogeometric approach. International Journal for Numerical Methods in Engineering, Wiley Online Library, v. 91, n. 6, p. 571-603, 2012.

THOMAS, D. C.; SCOTT, M. A. Isogeometric analysis based on t-splines. In: Isogeometric Methods for Numerical Simulation. [S.l.]: Springer, 2015. p. 205-232.

THOMAS, D. C. et al. Bézier projection: a unified approach for local projection and quadrature-free refinement and coarsening of nurbs and t-splines with particular application to isogeometric design and analysis. Computer Methods in Applied Mechanics and Engineering, Elsevier, v. 284, p. 55-105, 2015.

TIMOSHENKO, S. Theory of elasticity. 1st. Mcgraw-Hill Book Company, Inc.; New York, 1934.

TYRTYSHNIKOV, E. Mosaic-skeleton approximations. Calcolo, Springer, v. 33, n. 1-2, p. 47-57, 1996.

UBESSI, C. J. B. Método dos elementos de contorno para elasticidade linear 3d com avaliação direta das integrais singulares. Dissertação - UFRGS, 2014.

VANRAES, E. Powell-sabin splines and multiresolution techniques. status: published, 2004.

VEIGA, L. B. da et al. Analysis-suitable t-splines are dual-compatible. Computer methods in applied mechanics and engineering, Elsevier, v. 249, p. 42-51, 2012.

VEIGA, L. B. da et al. Analysis-suitable t-splines of arbitrary degree: definition, linear independence and approximation properties. Mathematical Models and Methods in Applied Sciences, World Scientific, v. 23, n. 11, p. 1979-2003, 2013.

VERHOOSEL, C. V. et al. An isogeometric approach to cohesive zone modeling. International Journal for Numerical Methods in Engineering, Wiley Online Library, v. 87, n. 1-5, p. 336-360, 2011. 
VERHOOSEL, C. V. et al. An isogeometric analysis approach to gradient damage models. International Journal for Numerical Methods in Engineering, Wiley Online Library, v. 86, n. 1, p. 115-134, 2011.

VORST, H. A. Van der; VUIK, C. The superlinear convergence behaviour of gmres. Journal of computational and applied mathematics, Elsevier, v. 48, n. 3, p. 327-341, 1993.

VUONG, A.-V. Adaptive Hierarchical Isogeometric Finite Element Methods. [S.1.]: Springer Science \& Business Media, 2012.

WANG, D.; ZHANG, H. A consistently coupled isogeometric-meshfree method. Computer Methods in Applied Mechanics and Engineering, Elsevier, v. 268, p. 843-870, 2014.

WANG, H. et al. Polycube splines. Computer-Aided Design, Elsevier, v. 40, n. 6, p. 721-733, 2008.

WANG, H.; YAO, Z. A new fast multipole boundary element method for large scale analysis of mechanical properties in 3d particle-reinforced composites. Comput. Model. Eng. Sci, v. 7, n. 1, p. 85-95, 2005.

WANG, Y.; BENSON, D. Multi-patch nonsingular isogeometric boundary element analysis in 3d. Computer Methods in Applied Mechanics and Engineering, Elsevier, v. 293, p. 71-91, 2015.

WROBEL, L.; ALIABADI, M. The Boundary Element Methods in Engineering. [S.l.]: McGraw-Hill College, 2002.

WROBEL, L. C. The Boundary Element Method, and Volume 1, and Applications in Thermo-Fluids and Acoustics. [S.l.]: wiley, 2002.

ZHANG, L.; NODERA, T. A new adaptive restart for gmres $(m)$ method. ANZIAM Journal, v. 46, p. 409-425, 2005.

ZHANG, Y.; GONG, Y.; GAO, X. Calculation of 2d nearly singular integrals over high-order geometry elements using the sinh transformation. Engineering Analysis with Boundary Elements, Elsevier, v. 60, p. 144-153, 2015.

ZWILLINGER, D. Handbook of differential equations. [S.l.]: Gulf Professional Publishing, 1998. v. 1. 